\title{
MODELING THE EFFECT OF TEMPERATURE VARIABILITY ON MALARIA CONTROL STRATEGIES
}

\author{
SAlisu M. Garba* And Usman A. Danbaba
}

\begin{abstract}
In this study, a non-autonomous (temperature dependent) and autonomous (temperature independent) models for the transmission dynamics of malaria in a population are designed and rigorously analysed. The models are used to assess the impact of temperature changes on various control strategies. The autonomous model is shown to exhibit the phenomenon of backward bifurcation, where an asymptotically-stable disease-free equilibrium (DFE) co-exists with an asymptotically-stable endemic equilibrium when the associated reproduction number is less than unity. This phenomenon is shown to arise due to the presence of imperfect vaccines and disease-induced mortality rate. Threshold quantities (such as the basic offspring number, vaccination and host type reproduction numbers) and their interpretations for the models are presented. Conditions for local asymptotic stability of the disease-free solutions are computed. Sensitivity analysis using temperature data obtained from Kwazulu Natal Province of South Africa [K. Okuneye and A.B. Gumel. Mathematical Biosciences 287 (2017) 72-92] is used to assess the parameters that have the most influence on malaria transmission. The effect of various control strategies (bed nets, adulticides and vaccination) were assessed via numerical simulations.
\end{abstract}

Mathematics Subject Classification. 37C75, 92D30.

Received November 16, 2019. Accepted November 1, 2020.

\section{INTRODUCTION}

Malaria is an important mosquito borne disease with a global distribution and significant public health burden. It is a life-threatening infection that is caused by Plasmodium parasite, spread and sustained through bites by female Anopheles mosquitoes on susceptible and infected humans [42]. There are more than hundred species of Plasmodium that can infect different animal species such as reptiles, birds and various mammals [38], among which five species (P. falciparum, P. vivax, P. ovale, $P$. malariae, and $P$. knowlesi) specifically cause human infections $[17,42]$. Out of the aforementioned species, $P$. vivax (which is the dominant malaria parasite outside of sub-Saharan Africa) and P. falciparum (the most prevalent malaria parasite on the African continent and responsible for most malaria-related deaths globally) were responsible for 891,000 deaths globally within the period 2015-2016 [42].

The most important environmental variables that affect mosquito population are suitable temperature and appropriate aquatic breeding sites. Temperature affects both survival and development rate of mosquitoes while

Keywords and phrases: Malaria, vector control, sensitivity analysis, offspring number, reproduction ratio.

Department of Mathematics and Applied Mathematics, University of Pretoria, Pretoria 0002, South Africa.

* Corresponding author: Salisu.Garba@up.ac.za

(C) The authors. Published by EDP Sciences, 2020

This is an Open Access article distributed under the terms of the Creative Commons Attribution License (https://creativecommons.org/licenses/by/4.0), which permits unrestricted use, distribution, and reproduction in any medium, provided the original work is properly cited. 
surface wetness limits the population size of aquatic mosquitoes [37]. In addition, temperature is a key determinant of environmental suitability for transmission of malaria in human. The spatial limits of the distribution and seasonal activities of malaria are sensitive to climate factors, as well as the local capacity to control the disease [8]. The dynamics and distribution of malaria strongly depend on the interplay between the parasite, the mosquitoes and the environment [28,34], it has recently been shown that mosquito and parasite biology are influenced not only by average temperature, but also by the extent of the daily temperature variation [5]. At the extremes, temperature regimes constrain the geographical extent of the disease and, within this envelope, contribute to determining its intensity [22]. These constraints are temporally dynamic, with fluctuations in transmission suitability and intensity driven by seasonal and inter-annual temperature cycles. The importance of temperature as an environmental determinant of malaria endemicity arises from a series of effects on the life cycles of the plasmodium parasite and anopheles mosquitoes [22].

Although, there have been tremendous success in the reduction of malaria cases especially in Africa, nevertheless, mortality due to the disease incidences still remains high in comparison to other infections. Over the years, there have been several initiatives aimed at ending malaria cases, some of which include WHO's Roll Back Malaria program, the Multilateral Initiative in Malaria, the Medicines for Malaria Venture, the Malaria Vaccine Initiative, and the Global Fund to Fight AIDS, TB and Malaria, which supports the implementation of prevention and treatment programs [38]. In fact, there is no single way of preventing malaria, however, there are a number of ways to decrease the transmission of the disease which include the use of treated nets, as well as the use of larvicides and adulticides to clear mosquito breeding sites and kill adult mosquitoes, respectively. Although there is no specific effective vaccine for malaria at the moment, a number of candidate vaccines targeting different stages of the malaria parasite life-cycle have been developed or are currently under development, in particular, RTS,S/AS01 is a strong candidate for the prevention of Plasmodium falciparum infection, in fact phase 3 trials of the vaccine has been completed [41]. Predicting the public health impact of a candidate malaria vaccine requires using clinical trial data to estimate the vaccine's efficacy profile, initial efficacy after vaccination and the pattern through which the vaccine efficacy wanes over time. With an estimated vaccine efficacy profile, the effects of vaccination on malaria transmission can be simulated with the aid of mathematical models [41].

A number of mathematical models have been developed in the literature to gain insights into the effects of temperature, climate change (or seasonality) in the transmission dynamics of mosquito borne diseases in a community, see for instance $[1-3,7,16,17,19,21,22,25-28,31,32,34,36,43]$. Eikenberry and Gumel [17] extensively review the idea of mathematical modeling of climate change in the transmission dynamics of malaria. Greenhalgh and Moreim [21] study SIRS epidemic model with general seasonal variation in the contact rate. Moreim and Greenhalgh [26] applies generalised periodic vaccination strategy to model for control of the spread and transmission of an infectious disease with seasonal varying contact rate. Dushoff [16] incorporate seasonality of birth rate into the standard deterministic SIR and SEIR epidemic models and identify parameter regions in which birth seasonality can be expected to have observable epidemiological effects. Bury et al. [7] develop a simple socio-climate model by coupling an Earth system model to a social dynamics model, they concluded that socio-climate models should be included in the ensemble of models used to project climate change. In particular, malaria has received lots of attention. Mordecai et al. [27] considered a non-linear response of mosquito and malaria parasite to temperature which are closely consistent with field data, the work which changed predictions on how temperature change affects malaria predicts optimal malaria transmission at $25^{\circ} \mathrm{C}$. A malaria transmission model with periodic birth rate and age structure for the vector population was rigorously analysed by Loy and Zhao [25]. The examination of the process via which parasite development within the mosquito (extrinsic incubation period) is expected to vary over time and space, depending on the diurnal temperature range and baseline mean temperature in Kenya and across Africa was presented by Blanford et al. [5]. Agusto et al. [2] consider a temperature-dependent deterministic model that gave some qualitative insights into the effects of temperature variability on malaria transmission dynamics, the model incorporates gradual increase in infection-acquired immunity via repeated exposure to malaria infection. The impact of variability in temperature and rainfall on the transmission dynamics of malaria in age-structured population, with the dynamics of immature and mature mosquitoes was also considered by Okuneye and Gumel [31]. A malaria model that qualitatively studied the effect of seasonal variations (wet and dry seasons) on the spread of malaria 
was introduced and analysed by Dembele et al. [13], the authors present an explicit formulation for the basic reproduction ratio and stability analysis of the disease free equilibrium. Olaniyi et al. [33] present malaria model that takes into account time-dependent control functions such as personal protection, treatments and mosquito reduction intervention strategies.

In this study, we consider both autonomous and non-autonomous deterministic models for the transmission dynamics of malaria with control in the presence of temperature variability. Notable feature of the model is that the host population is basically divided according to their vaccination status and the use of multiple control and prevention strategies. The model further assumed that recovered individuals do not become wholly susceptible. The paper is organized as follows. A non-autonomous malaria model is developed and some of its dynamical properties are discussed in Section 2. The autonomous model is analysed for its dynamical properties in Section 3. Endemic equilibrium and the backward bifurcation analysis of the autonomous model is presented in Section 4. Section 5 presents the analysis of the non-autonomous system. The effect of various control strategies is provided in Section 6. Sensitivity analysis and numerical simulations are presented in Section 7.

\section{MODEL FORMULATION}

The total human population at time $t$, denoted by $N_{H}(t)$, is divided into populations of vaccinated and non-vaccinated individuals. The sub-population of non-vaccinated individuals are further sub-divided into 5 mutually exclusive sub-population of wholly susceptible (without ever been infected) $\left(S_{U}(t)\right.$ ), susceptible after recovery $\left(W_{U}(t)\right)$, exposed $\left(E_{U}(t)\right)$, infected $\left(I_{U}(t)\right)$ and recovered $\left(R_{U}(t)\right)$ humans. Similarly, the sub-population of vaccinated individuals are sub-divided into wholly susceptible $\left(S_{V}(t)\right)$, susceptible after recovery $\left(W_{V}(t)\right)$, exposed $\left(E_{V}(t)\right)$, infected $\left(I_{V}(t)\right)$ and recovered $\left(R_{V}(t)\right)$ humans, so that the total human population at time $t$ is given by

$$
N_{H}(t)=S_{U}(t)+S_{V}(t)+W_{U}(t)+W_{V}(t)+E_{U}(t)+E_{V}(t)+I_{U}(t)+I_{V}(t)+R_{U}(t)+R_{V}(t) .
$$

In order to assess the potential effect of temperature dependent oviposition of mosquitoes, the population of mosquitoes are divided into aquatic and non-aquatic stages. The aquatic stage (which involves egg, larva and pupa) is represented by a single equation $\left(A_{M}(t)\right)$. The non-aquatic (adult) stage is further divided into susceptible $\left(M_{U}(t)\right)$, exposed $\left(M_{E}(t)\right)$ and infected $\left(M_{I}(t)\right)$ mosquitoes. so that the total adult mosquito population (in the non-aquatic stage) is given by

$$
N_{V}(t)=M_{U}(t)+M_{E}(t)+M_{I}(t) .
$$

Note that, in this study only female mosquitoes are considered as male mosquitoes are non-infectious. The model incorporates the use of larvicides (to clear aquatic mosquitoes) and adulticides (to kill matured mosquitoes). In either case, the death rates due to the use of larvicides and adulticides are proportional to successful rates of applications of larvicides and adulticides.

\subsection{Dynamics of humans}

The population of wholly susceptible humans is generated by birth (or immigration) at a constant rate $\Pi_{H}$. This population increases through the loss of vaccination-acquired immunity by wholly vaccinated individuals (at a waning rate $\omega_{V}$ ). It is decreased by vaccination (at a rate $\xi_{V}$ which move to the class of wholly vaccinated humans). Proportion of this individuals (in $S_{U}$ class) acquire malaria infection following effective contact with infectious mosquitoes in $M_{I}$ class at a temperature dependent rate $\lambda_{H}(T)$, given by

$$
\lambda_{H}(T)=\frac{\beta_{V H} M_{I}(t)}{N_{V}(t)}\left(1-\epsilon_{B} \alpha_{B}\right) a_{M}(T),
$$


where the parameter $\beta_{V H}$ is a transmission probability from infectious mosquitoes to susceptible humans. The parameter $a_{M}(T)$ is the temperature dependent biting rate of mosquitoes, $0<\epsilon_{B}<1$ represents efficacy rate of bed nets and $\alpha_{B}$ measure compliance rate in the use of bed nets (low values of $\alpha_{B}$ imply limited use of bed nets by the members of the public, whereas values of $\alpha_{B}$ close to one imply widespread use of bed nets). Therefore $\epsilon_{B} \alpha_{B}$ represents the use of insect repellents to minimize contacts with mosquitoes. Natural mortality occurs in all human classes at a rate $\mu_{H}$, so that

$$
\frac{\mathrm{d} S_{U}(t)}{\mathrm{d} t}=\Pi_{H}+\omega_{V} S_{V}(t)-\xi_{V} S_{U}(t)-\lambda_{H}(T) S_{U}(t)-\mu_{H} S_{U}(t)
$$

The population of a wholly vaccinated individuals $S_{V}$ is generated by vaccination of susceptible individuals at the rate $\xi_{V}$. This population decreases due to waning of vaccine (at the rate $\omega_{V}$ ), infection at the rate $\lambda_{H}(T)\left(1-\epsilon_{V}\right)$ (where $0<\epsilon_{V}<1$ is a vaccine efficacy) and by natural death (at the rate $\mu_{H}$ ). This gives

$$
\frac{\mathrm{d} S_{V}(t)}{\mathrm{d} t}=\xi_{V} S_{U}(t)-\lambda_{H}(T)\left(1-\epsilon_{V}\right) S_{V}(t)-\omega_{V} S_{V}(t)-\mu_{H} S_{V}(t)
$$

The populations of non-vaccinated $\left(W_{U}\right)$ and vaccinated $\left(W_{V}\right)$ susceptible individuals (who are partially immune due to prior infection) are generated following loss of partial immunity by recovered individuals that are non-vaccinated and vaccinated at the rates $\tau_{U}$ and $\tau_{V}$, respectively. These populations decrease by secondary infection at a reduced rate $\lambda_{H}(T)\left(1-\epsilon_{W}\right.$ ) (where $\epsilon_{W}$ is a protection rate due to prior malaria infection) and by natural death. So that

$$
\begin{aligned}
& \frac{\mathrm{d} W_{U}(t)}{\mathrm{d} t}=\tau_{U} R_{U}(t)-\lambda_{H}(T)\left(1-\epsilon_{W}\right) W_{U}(t)-\mu_{H} W_{U}(t), \\
& \frac{\mathrm{d} W_{V}(t)}{\mathrm{d} t}=\tau_{V} R_{V}(t)-\lambda_{H}(T)\left(1-\epsilon_{W}\right) W_{V}(t)-\mu_{H} W_{V}(t) .
\end{aligned}
$$

The populations of non-vaccinated exposed $\left(E_{U}\right)$ and vaccinated exposed $\left(E_{V}\right)$ individuals are generated by the infection of non-vaccinated $\left(S_{U}, W_{U}\right)$ and vaccinated $\left(S_{V}, W_{V}\right)$ individuals at the rates $\lambda_{H}, \lambda_{H}(T)\left(1-\epsilon_{W}\right)$ and $\lambda_{H}\left(1-\epsilon_{V}\right), \lambda_{H}(T)\left(1-\epsilon_{W}\right)$, respectively. These populations are reduced by progressing to non-vaccinated and vaccinated infectious classes at the rates $\sigma_{U}$ and $\sigma_{V}$, respectively, and by natural death. Thus

$$
\begin{aligned}
& \frac{\mathrm{d} E_{U}(t)}{\mathrm{d} t}=\lambda_{H}(T) S_{U}(t)+\lambda_{H}(T)\left(1-\epsilon_{W}\right) W_{U}(t)-\sigma_{U} E_{U}(t)-\mu_{H} E_{U}(t), \\
& \frac{\mathrm{d} E_{V}(t)}{\mathrm{d} t}=\lambda_{H}(T)\left(1-\epsilon_{V}\right) S_{V}(t)+\lambda_{H}(T)\left(1-\epsilon_{W}\right) W_{V}(t)-\sigma_{V} E_{V}(t)-\mu_{H} E_{V}(t) .
\end{aligned}
$$

The populations of non-vaccinated infectious $\left(I_{U}\right)$ and vaccinated infectious $\left(I_{V}\right)$ individuals are generated by progression of non-vaccinated and vaccinated exposed individuals to the infectious classes at the rates $\sigma_{U}$ and $\sigma_{V}$, respectively. These populations decreases by recovery at the rates $\gamma_{U}$ and $\gamma_{V}$, respectively, natural death and disease-induced death at the rates $\delta_{U}$ and $\delta_{V}$, respectively. This gives

$$
\begin{aligned}
& \frac{\mathrm{d} I_{U}(t)}{\mathrm{d} t}=\sigma_{U} E_{U}(t)-\gamma_{U} I_{U}(t)-\delta_{U} I_{U}(t)-\mu_{H} I_{U}(t), \\
& \frac{\mathrm{d} I_{V}(t)}{\mathrm{d} t}=\sigma_{V} E_{V}(t)-\gamma_{V} I_{V}(t)-\delta_{V} I_{V}(t)-\mu_{H} I_{V}(t) .
\end{aligned}
$$

The populations of non-vaccinated recovered $\left(R_{U}\right)$ and vaccinated recovered $\left(R_{V}\right)$ individuals are generated by the recovery of non-vaccinated and vaccinated infectious individuals at the rates $\gamma_{U}$ and $\gamma_{V}$, respectively. 
The populations are reduces by loss of partial immunity at the rates $\tau_{U}$ and $\tau_{V}$, respectively, and natural death. So that

$$
\begin{aligned}
& \frac{\mathrm{d} R_{U}(t)}{\mathrm{d} t}=\gamma_{U} I_{U}(t)-\tau_{U} R_{U}(t)-\mu_{H} R_{U}(t), \\
& \frac{\mathrm{d} R_{V}(t)}{\mathrm{d} t}=\gamma_{V} I_{V}(t)-\tau_{V} R_{V}(t)-\mu_{H} R_{V}(t) .
\end{aligned}
$$

\subsection{Dynamics of mosquitoes}

In the presence of intervention (using larvicides), the population of aquatic mosquitoes (eggs, larvae and pupae) increases through oviposition by reproductive mosquitoes at a temperature dependent rate $\phi_{A}(T)$ $\left(1-\alpha_{L} \epsilon_{L}\right)$, where $\alpha_{L}$ is a rate of applying larvicides and $\epsilon_{L}$ is an efficacy of larvicides (so that $\alpha_{L} \epsilon_{L}=c_{L}$ accounts for effectiveness of larvicides). The growth of aquatic mosquitoes is moderated by a constant environmental carrying capacity $K$. This population decreases by maturation at a temperature dependent rate $\sigma_{A}(T)$, die naturally and due to the use of larvicides at a rate $\mu_{A}(T)\left(1+\alpha_{L} \epsilon_{L}\right)$, where $\mu_{A}(T)$ is a temperature dependent death rate. Thus

$$
\frac{\mathrm{d} A_{M}(t)}{\mathrm{d} t}=\phi_{A}(T)\left(1-\alpha_{L} \epsilon_{L}\right)\left(1-\frac{A_{M}(t)}{K}\right) N_{V}(t)-\sigma_{A}(T) A_{M}(t)-\mu_{A}(T)\left(1+\alpha_{L} \epsilon_{L}\right) A_{M}(t) .
$$

The population of susceptible adult female mosquitoes $\left(M_{U}(t)\right)$ is generated by maturation of aquatic mosquitoes at the temperature dependent rate $\sigma_{A}(T)$. It decreases by acquiring infection following a substantial contact with an infectious human at a temperature dependent infection rate $\lambda_{V}(T)$, given by

$$
\lambda_{V}(T)=\frac{\beta_{H V}}{N_{H}(t)}\left(1-\epsilon_{B} \alpha_{B}\right) a_{M}(T)\left[I_{U}(t)+\eta_{I} I_{V}(t)+\eta_{U} R_{U}+\eta_{V} R_{V}\right]
$$

where, $\beta_{H V}$ is the probability of infection from infectious humans to susceptible mosquitoes. The parameters $\eta_{I}$, $\eta_{U}$ and $\eta_{V}$ are modification parameters which account for the reduction in the infectivity of individuals in $I_{V}, R_{U}$ and $R_{V}$ classes in comparison to those in $I_{U}$ class, respectively. Similarly, the population is further decreases at a rate $\mu_{V}(T)\left(1+\alpha_{A} \epsilon_{A}\right)$, where $\mu_{V}(T)$ is a temperature dependent death in the absence of intervention, $\alpha_{A}$ is a rate of applying adulticides and $\epsilon_{A}$ is an efficacy of adulticides (so that $\alpha_{A} \epsilon_{A}=c_{A}$ accounts for effectiveness of indoor residual spraying). Thus

$$
\frac{\mathrm{d} M_{U}(t)}{\mathrm{d} t}=\sigma_{A}(T) A_{M}(t)-\lambda_{V}(T) M_{U}(t)-\mu_{V}(T)\left(1+\alpha_{A} \epsilon_{A}\right) M_{U}(t) .
$$

The population of exposed mosquitoes in the $M_{E}(t)$ class is generated by the infection of adult mosquitoes in the $M_{U}(t)$ class at the rate $\lambda_{V}(T)$. This population decreases by progression to infectious class at a temperature dependent rate $\sigma_{M}(T)$ and die naturally and due to the use of adulticides at the rate $\mu_{V}(T)\left(1+\alpha_{A} \epsilon_{A}\right)$. Hence

$$
\frac{\mathrm{d} M_{E}(t)}{\mathrm{d} t}=\lambda_{V}(T) M_{U}(t)-\sigma_{M}(T) M_{E}(t)-\mu_{V}(T)\left(1+\alpha_{A} \epsilon_{A}\right) M_{E}(t)
$$

Finally, the population of infectious mosquitoes in the $M_{I}(t)$ class is generated by progression of mosquitoes in the $M_{E}(t)$ class to $M_{I}(t)$ class at the temperature dependent rate $\sigma_{M}(T)$. It decreases due to natural death 
and the use of adulticides at the rate $\mu_{V}(T)\left(1+\alpha_{A} \epsilon_{A}\right)$. This gives

$$
\frac{\mathrm{d} M_{I}(t)}{\mathrm{d} t}=\sigma_{M}(T) M_{E}(t)-\mu_{V}(T)\left(1+\alpha_{A} \epsilon_{A}\right) M_{I}(t) .
$$

Thus, the above formulations for human and mosquito dynamics are represented by the following nonautonomous deterministic system of non-linear differential equations (a flow diagram of the model is depicted in Fig. 1 and the state variables and parameters of the model are described in Tabs. 1 and 2):

$$
\begin{aligned}
& \frac{\mathrm{d} S_{U}(t)}{\mathrm{d} t}=\Pi_{H}+\omega_{V} S_{V}(t)-\xi_{V} S_{U}(t)-\lambda_{H}(T) S_{U}(t)-\mu_{H} S_{U}(t) \\
& \frac{\mathrm{d} S_{V}(t)}{\mathrm{d} t}=\xi_{V} S_{U}(t)-\lambda_{H}(T)\left(1-\epsilon_{V}\right) S_{V}(t)-\omega_{V} S_{V}(t)-\mu_{H} S_{V}(t), \\
& \frac{\mathrm{d} W_{U}(t)}{\mathrm{d} t}=\tau_{U} R_{U}(t)-\lambda_{H}(T)\left(1-\epsilon_{W}\right) W_{U}(t)-\mu_{H} W_{U}(t) \\
& \frac{\mathrm{d} W_{V}(t)}{\mathrm{d} t}=\tau_{V} R_{V}(t)-\lambda_{H}(T)\left(1-\epsilon_{W}\right) W_{V}(t)-\mu_{H} W_{V}(t) \\
& \frac{\mathrm{d} E_{U}(t)}{\mathrm{d} t}=\lambda_{H}(T) S_{U}(t)+\lambda_{H}(T)\left(1-\epsilon_{W}\right) W_{U}(t)-\sigma_{U} E_{U}(t)-\mu_{H} E_{U}(t), \\
& \frac{\mathrm{d} E_{V}(t)}{\mathrm{d} t}=\lambda_{H}(T)\left(1-\epsilon_{V}\right) S_{V}(t)+\lambda_{H}(T)\left(1-\epsilon_{W}\right) W_{V}(t)-\sigma_{V} E_{V}(t)-\mu_{H} E_{V}(t), \\
& \frac{\mathrm{d} I_{U}(t)}{\mathrm{d} t}=\sigma_{U} E_{U}(t)-\gamma_{U} I_{U}(t)-\delta_{U} I_{U}(t)-\mu_{H} I_{U}(t) \\
& \frac{\mathrm{d} I_{V}(t)}{\mathrm{d} t}=\sigma_{V} E_{V}(t)-\gamma_{V} I_{V}(t)-\delta_{V} I_{V}(t)-\mu_{H} I_{V}(t) \\
& \frac{\mathrm{d} R_{U}(t)}{\mathrm{d} t}=\gamma_{U} I_{U}(t)-\tau_{U} R_{U}(t)-\mu_{H} R_{U}(t) \\
& \frac{\mathrm{d} R_{V}(t)}{\mathrm{d} t}=\gamma_{V} I_{V}(t)-\tau_{V} R_{V}(t)-\mu_{H} R_{V}(t), \\
& \frac{\mathrm{d} A_{M}(t)}{\mathrm{d} t}=\phi_{A}(T)\left(1-\alpha_{L} \epsilon_{L}\right)\left(1-\frac{A_{M}(t)}{K}\right) N_{V}(t)-\sigma_{A}(T) A_{M}(t)-\mu_{A}(T)\left(1+\alpha_{L} \epsilon_{L}\right) A_{M}(t), \\
& \frac{\mathrm{d} M_{U}(t)}{\mathrm{d} t}=\sigma_{A}(T) A_{M}(t)-\lambda_{V}(T) M_{U}(t)-\mu_{V}(T)\left(1+\alpha_{A} \epsilon_{A}\right) M_{U}(t), \\
& \frac{\mathrm{d} M_{E}(t)}{\mathrm{d} t}=\lambda_{V}(T) M_{U}(t)-\sigma_{M}(T) M_{E}(t)-\mu_{V}(T)\left(1+\alpha_{A} \epsilon_{A}\right) M_{E}(t), \\
& \frac{\mathrm{d} M_{I}(t)}{\mathrm{d} t}=\sigma_{M}(T) M_{E}(t)-\mu_{V}(T)\left(1+\alpha_{A} \epsilon_{A}\right) M_{I}(t)
\end{aligned}
$$

For simulation purpose, a generalized temperature function given by

$$
T(t)=T_{0}\left[1+T_{1} \cos \left(\frac{2 \pi}{365}(\omega t+\phi)\right)\right]
$$

will be used, where $T_{0}$ is the mean annual temperature, $T_{1}$ represents the variation about the mean, $\omega$ measures the periodicity of the function and $\phi$ is the phase shift of the function. Therefore the time dependent temperature $T=T(t)$, the temperature dependent parameters $\phi_{A}(T), \sigma_{A}(T), \mu_{A}(T), \mu_{V}(T), a_{M}(T)$ and $\sigma_{M}(T)$ are continuous, bounded, positive and $\omega$-periodic functions. That is they belong to $L_{+}^{\infty}\left(0, \omega, \mathbb{R}_{+}\right)$.

Using similar argument to those in $[10-12,18,25,29]$ (and some of the references therein) and the basic fact that for mosquito-borne diseases (such as malaria), the total number of bites made by mosquitoes must equal 


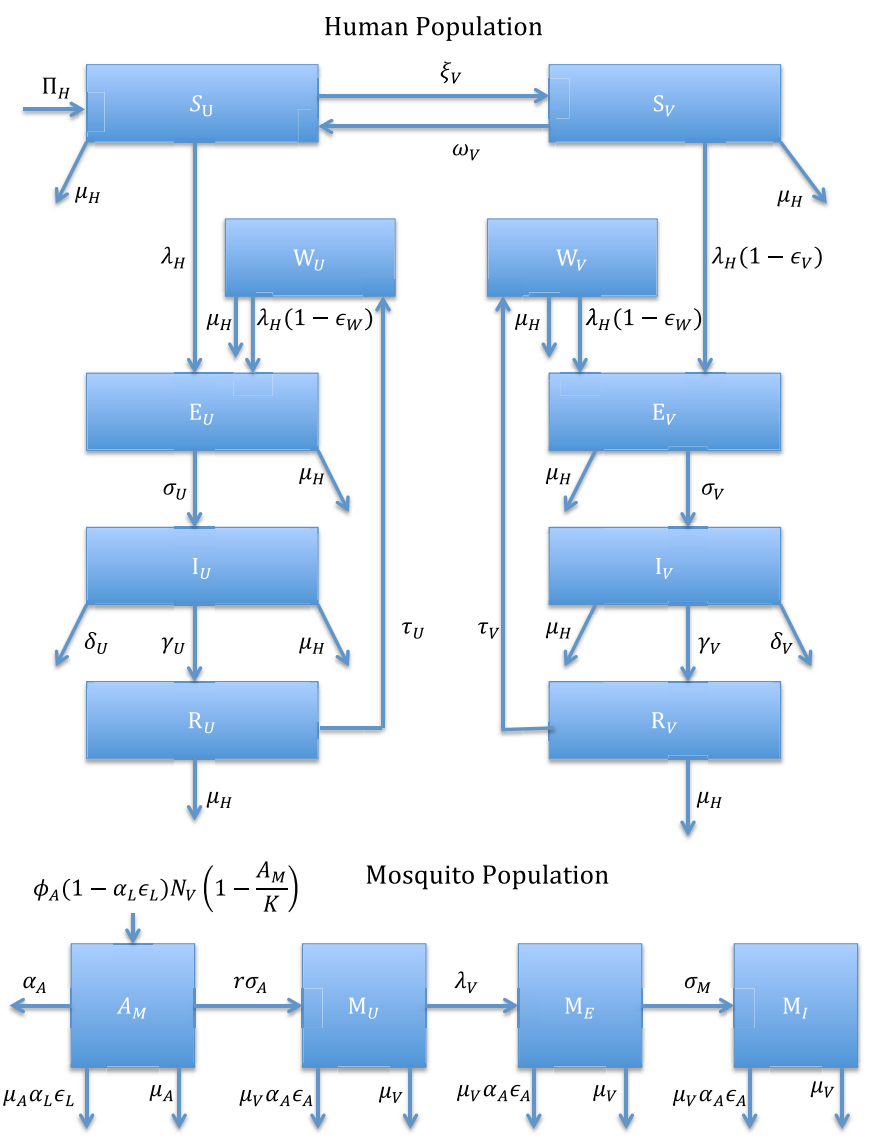

FIGURE 1. Schematic diagram of the basic model (2.1).

the total number of bites received by humans. Thus, for the number of bites to be conserved, the following equation must hold

$$
\beta_{H V} N_{V}=\beta_{V H}\left(N_{H}, N_{V}\right) N_{H},
$$

so that

$$
N_{V}=\frac{\beta_{V H}\left(N_{H}, N_{V}\right)}{\beta_{H V}} N_{H}
$$

Thus, the force of infection in human populations is now given by

$$
\lambda_{H}(T)=\frac{\beta_{H V} M_{I}(t)}{N_{H}(t)}\left(1-\epsilon_{B} \alpha_{B}\right) a_{M}(T) .
$$

The model extends (in certain ways) some malaria transmissions models in the literature, such as those in $[1-3,17,22,25,28,31,34,43]$, by inter alia:

(I) Assuming that recovered individuals do not revert to wholly-susceptible class because they enjoy reduced susceptibility to new malaria infection $[2,3]$; 
(II) Incorporating vaccination and the use of treated bed nets in humans (this was not considered in $[2,3,31]$ );

(III) Including both the use of larvicides and adulticides in mosquito populations (this was not considered in $[1-3,17,22,25,28])$

(IV) Dividing human population into compartments based on malaria infection in line with their vaccination status (this was not considered in $[2,3,31]$ );

(V) Considering a reduced disease induced death rate, faster recovery rate and slower waning of immunity for vaccinated humans, i.e $\delta_{V} \leq \delta_{U}, \gamma_{V} \geq \gamma_{U}$ and $\tau_{V} \leq \tau_{U}$, this was also not considered in [1-3, 17, 22, 25, $28,34]$;

(VI) Incorporating the effect of endemicity of malaria by differentiating wholly susceptible from susceptible with prior infection, this was not considered in [2, 3, 22, 25, 28, 33, 34, 43].

\subsection{Temperature dependent parameters}

Temperature is known to directly affects vector borne diseases in host; insects are poikilothermic and hence their internal temperature is greatly influenced by environmental temperature, which affect their physiology, as well as exposing the pathogen they carry to environmental temperature [34]. Using the formulations in $[6,27,31,36]$, the temperature dependent parameters of malaria model $(2.1)$ are defined either as Briere or Quadratic functions as follows. The temperature dependent:

1. Oviposition rate $\left(\phi_{A}(T)\right)$ of mosquitoes is given by

$$
\phi_{A}(T)=-0.153 T^{2}+8.61 T-0.487 .
$$

2. Maturation rate $\left(\sigma_{A}(T)\right)$ of aquatic mosquitoes is given by

$$
\sigma_{A}(T)=0.000111(T-14.7) \sqrt{34-T}, \quad(0 \leq T \leq 34) .
$$

3. Death rate of aquatic mosquitoes is obtained from the formulation in [36] as

$$
\mu_{A}=0.0025 T^{2}-0.094 T+0.9 .
$$

4. Daily survival probability of adult mosquitoes (a function of the adult mosquito mortality rate) also follows from $[27]$ as

$$
\rho_{M}(T)=e^{-\mu_{V}(T)}=-0.000828 T^{2}+0.0367 T+0.522,
$$

so that the temperature dependent mortality rate of adult mosquitoes $\left(\mu_{V}(T)\right)$ is given by

$$
\mu_{V}(T)=-\ln \left(\rho_{M}(T)\right)=-\ln \left(-0.000828 T^{2}+0.0367 T+0.522\right) .
$$

5. Biting rate $\left(a_{M}\right)$ and parasite development rate $\left(\sigma_{M}(T)\right)$ of adult mosquitoes are respectively given by

$$
a_{M}(T)=-0.000203 T(T-11.7) \sqrt{42.3-T}, \quad(0 \leq T \leq 42.3)
$$

and

$$
\sigma_{M}(T)=0.000111(T-14.7) \sqrt{34.4-T}, \quad(0 \leq T \leq 34.4) .
$$

6. Finally the temperature dependent vector competence defined as the product of the proportion of the bites by infective mosquitoes that infect susceptible humans and the bites by susceptible mosquitoes on 

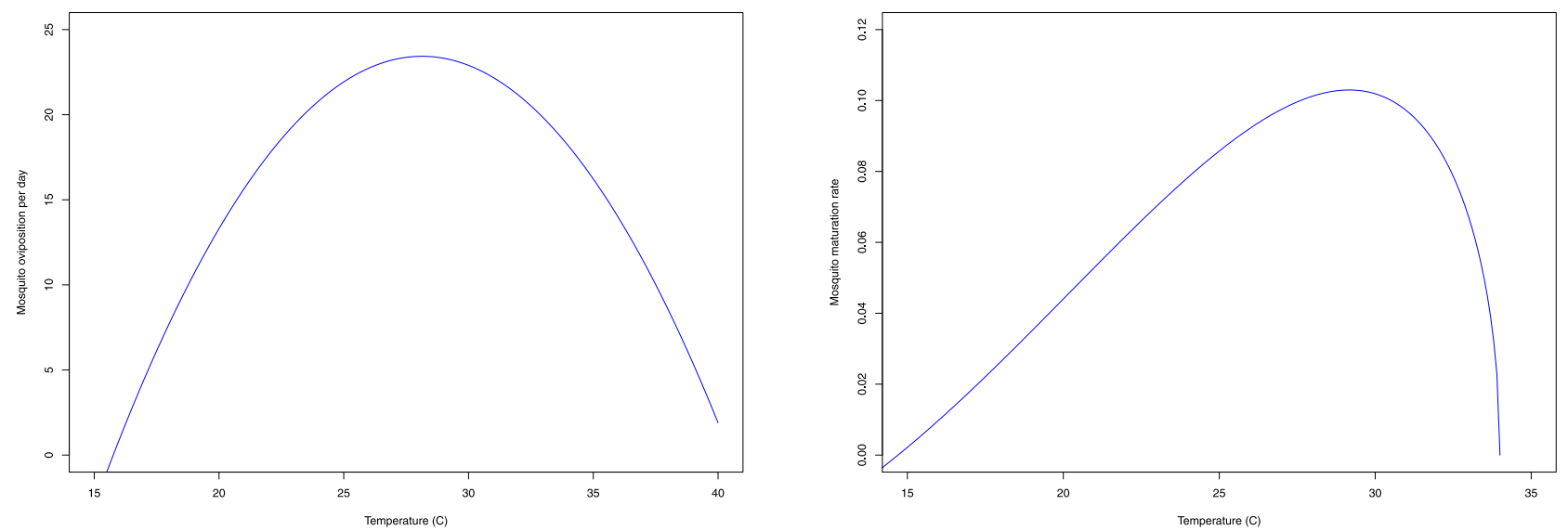

Figure 2. Profile of temperature $(T)$ dependent oviposition rate of adult mosquitoes given by $\phi_{A}(T)=-0.153 T^{2}+8.61 T-97.7$ and maturation rate of aquatic mosquitoes given by $\sigma_{A}(T)=$ $0.000111 T(T-14.7) \sqrt{34-T}$.
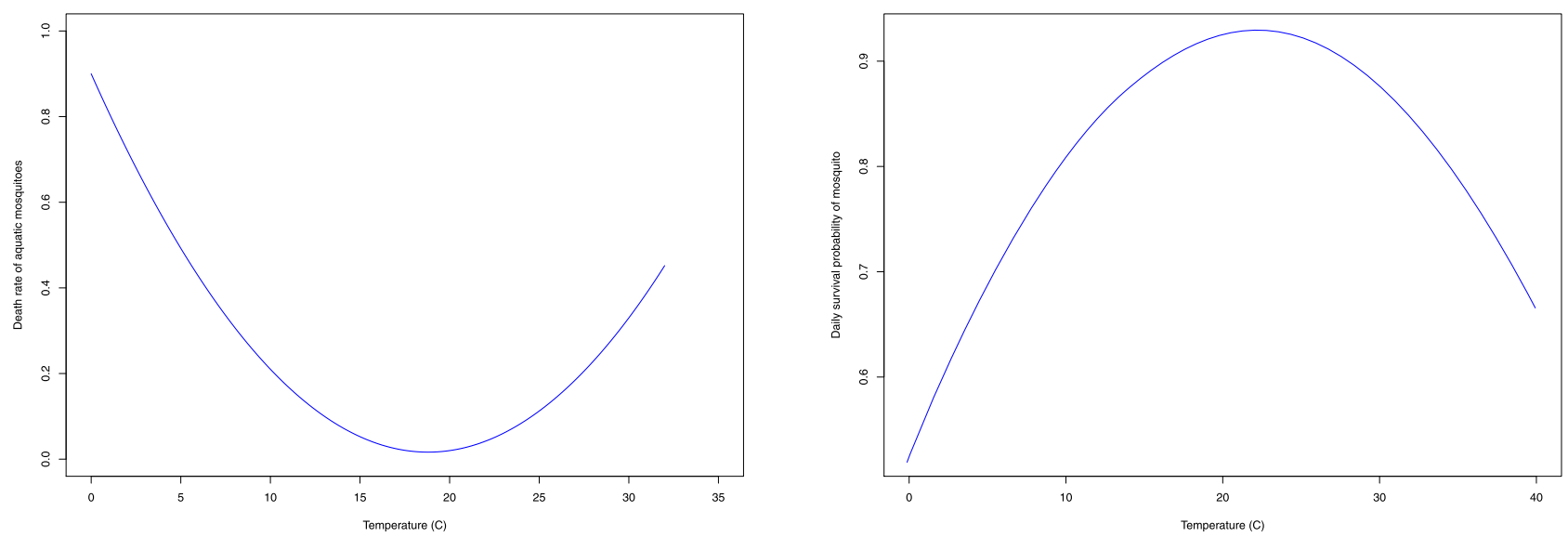

Figure 3. Profile of temperature $(T)$ dependent death rate of aquatic mosquitoes given by $\mu_{A}(T)=0.0025 T^{2}-0.09 T+0.9$ and daily survival probability of adult mosquitoes given by $\sigma_{M}(T)=-0.000828 T^{2}+0.0367 T+0.522$.

infectious humans that infect susceptible mosquitoes [27] is given by

$$
V(T)=-0.54 T^{2}+25.2 T-206
$$

The graphical representations of the temperature dependent parameters are presented in Figures 2, 3, 4 and 5 .

\subsection{Basic properties of the model $(2.1)$}

The basic dynamical properties of the non-autonomous system given by (2.1) is explored. Adding the first ten equations of the model (2.1) gives

$$
\frac{\mathrm{d} N_{H}}{\mathrm{~d} t}=\Pi_{H}-\mu_{H} N_{H}-\delta_{U} I_{U}-\delta_{V} I_{V}
$$





Figure 4. Profile of temperature $(T)$ dependent death rate of adult mosquitoes given by $\mu_{V}(T)=-\ln \left(-0.000828 T^{2}+0.0367 T+0.522\right)$ and biting rate of adult mosquitoes given by $a_{M}(T)=0.000203 T(T-11.7) \sqrt{42.3-T}$.


Figure 5. Profile of temperature $(T)$ dependent parasite development rate in mosquitoes given by $\sigma_{M}(T)=0.000111 T(T-14.7) \sqrt{34.4-T}$ and vectorial capacity of mosquitoes given by $V(T)=-0.54 T^{2}+25.2 T-206$.

Since

$$
\frac{\mathrm{d} N_{H}}{\mathrm{~d} t} \leq \Pi_{H}-\mu_{H} N_{H}
$$

it follows that $\mathrm{d} N_{H} / \mathrm{d} t<0$ if $N_{H}(t)>\Pi_{H} / \mu_{H}$. Thus, a standard comparison theorem can be used to show that $N_{H}(t) \leq N_{H}(0) e^{-\mu_{H}}+\frac{\Pi_{H}}{\mu_{H}}\left(1-e^{-\mu_{H}}\right)$, which is bounded. Furthermore, letting $\mu_{M}(T)=\min \left\{\mu_{A}(T), \mu_{V}(T)\right\}$, and $c_{M}=\min \left\{\alpha_{L} \epsilon_{L}, \alpha_{A} \epsilon_{A}\right\}$, the total mosquito population (in both aquatic and non-aquatic stages) satisfies

$$
\frac{\mathrm{d} N_{V}}{\mathrm{~d} t} \leq \phi_{A}(T)\left(1-c_{M}\right)\left(1-\frac{A_{M}(t)}{K}\right) N_{V}(t)-\mu_{M}(T)\left(1+c_{M}\right) N_{V} .
$$


Using similar approach to that of [25] and Gronwall's lemma, the mosquito population has a globallyasymptotically periodic solution satisfying

$$
\begin{aligned}
N_{V}^{*}(t)= & e^{-\int_{0}^{t} \mu_{M}(s)\left(1+c_{M}\right) \mathrm{d} s} \times\left[\int_{0}^{t}\left\{\phi_{A}(s)\left(1-c_{M}\right)\left(1-\frac{A_{M}(s)}{K}\right) N_{V}(s) e^{\int_{0}^{s} \mu_{M}(k)\left(1+c_{M}\right) \mathrm{d} k}\right\} \mathrm{d}(s)\right. \\
& \left.+\frac{\int_{0}^{\omega}\left\{\phi_{A}(s)\left(1-c_{M}\right)\left(1-\frac{A_{M}(s)}{K}\right) N_{V}(s) e^{\int_{0}^{u} \mu_{M}(n)\left(1+c_{M}\right) \mathrm{d} n}\right\} \mathrm{d}(s)}{e^{\int_{0}^{\omega} \mu_{M}(n)\left(1+c_{M}\right) \mathrm{d} n}-1}\right] .
\end{aligned}
$$

Also from [25], it is assumed that the mosquito population stabilizes at a periodic state, thus for the continuous, bounded, positive and $\omega$-periodic functions $\phi_{A}(T), \sigma_{A}(T), \mu_{A}(T), \mu_{V}(T), a_{M}(T)$ and $\sigma_{M}(T)$, there exist a positive number $h_{0}$, such that

$$
\phi_{A}(T)\left(1-c_{M}\right)\left(1-\frac{A_{M}(t)}{K}\right) L(t)-\mu_{M}(T)\left(1+c_{M}\right) L<0, \quad \text { for all } L \geq h_{0} .
$$

Lemma 2.1. Consider the non-autonomous model (2.1) with non-negative initial condition for all $t \geq 0$. Then for any $x \in C\left([0], \mathbb{R}_{+}^{14}\right)$, the model has a unique non-negative solution through $x$ that is ultimately bounded and uniformly bounded.

Let $X \in C\left([0], \mathbb{R}_{+}^{14}\right)$ and $G(t, x):=B(X) X+Z$ be the right hand side of $(2.1)$, where $X=$ $\left(S_{U}, S_{V}, W_{U}, W_{V}, E_{U}, E_{V}, I_{U}, I_{V}, R_{U}, R_{V}, A_{M}, M_{U}, M_{E}, M_{I}\right)^{T}, B(X)$ is the $14 \times 14$ matrix of coefficients of the non-constant part of $(2.1)$, while $Z=\left(\Pi_{H} / \mu_{H}, 0,0,0,0,0,0,0,0,0,0,0,0,0\right)^{T}$.

It is clear that for all $x \in C\left([0], \mathbb{R}_{+}^{14}\right), G(t, x)$ is continuous, and Lipschitzian in $x$, in addition, $\frac{\partial G_{j}(t, x)}{\partial x_{i}}>0$ for all $i=1,2, \ldots 14$ whenever $x_{i} \geq 0$ and $x_{j}=0$, thus existence, uniqueness and positivity of solution through $(0, X)$ is guaranteed [25]. Following the approach of $[14,15,31]$ and the fact that $B(X)$ is Metzler and $Z>0$, the system is positively invariant in $C\left([0], \mathbb{R}_{+}^{14}\right)$.

Moreover it follows from $(2.5)$ and $(2.7)$, that $\limsup _{t \longrightarrow \infty} N_{H}(t) \leq \frac{\Pi_{H}}{\mu_{H}}$ and $\limsup _{t \longrightarrow \infty}\left(A_{M}+M_{U}+M_{E}+M_{I}-\right.$ $\left.N_{V}^{*}(t)\right) \leq 0$, where $N_{V}^{*}(t)$ is the unique $\omega$-periodic solution defined by (2.6). Furthermore, $\frac{\mathrm{d} N_{H}(t)}{\mathrm{d} t}<0$ whenever $N_{H}(t)>\frac{\Pi_{H}}{\mu_{H}}$ and $\frac{\mathrm{d} N_{V}(t)}{\mathrm{d} t}<0$ if $N_{V}(t)>h_{0}$. Hence all solutions for the system given by (2.1) are ultimately and uniformly bounded [25].

\section{Analysis of the autonomous model}

In this section, we analyse the dynamics of the autonomous form of the model (2.1). That is the case when the model parameters are temperature independent. Thus

$$
a_{M}(T)=a_{M}, \quad \phi_{A}(T)=\phi_{A}, \quad \sigma_{A}(T)=\sigma_{A}, \quad \mu_{A}(T)=\mu_{A}, \quad \sigma_{M}(T)=\sigma_{M}, \quad \mu_{V}(T)=\mu_{V} .
$$

We first of all analysed mosquito-only model in the absence of interaction with humans for its basic dynamical features.

\subsection{Mosquito-only population model}

In this section, we carry out analysis of mosquito-only population model in the absence of interaction with humans and no intervention (adulticide or larvicide) is apply. In the absence of humans, the model (2.1), reduces 
to the following mosquito-only system:

$$
\begin{aligned}
& \frac{\mathrm{d} A_{M}(t)}{\mathrm{d} t}=\phi_{A}\left(1-\frac{A_{M}(t)}{K}\right) N_{V}(t)-\left(\sigma_{A}+\mu_{A}\right) A_{M}(t), \\
& \frac{\mathrm{d} M_{U}(t)}{\mathrm{d} t}=\sigma_{A} A_{M}(t)-\mu_{V} M_{U}(t) .
\end{aligned}
$$

The system (3.1) has a threshold quantity called the basic offspring number denoted by $N_{0}$, given by

$$
N_{0}=\frac{\phi_{A} \sigma_{A}}{\left(\sigma_{A}+\mu_{A}\right) \mu_{V}}
$$

The threshold quantity $\left(N_{0}\right)$ can be interpreted as follows: The average time spent by mosquito in the aquatic stage is given by $1 /\left(\sigma_{A}+\mu_{A}\right)$, where $\sigma_{A}$ is the rate at which aquatic mosquitoes develop into an adult mosquito, so that the probability that an aquatic mosquito develop into an adult female mosquito is given by

$$
\frac{\sigma_{A}}{\sigma_{A}+\mu_{A}}
$$

In the absence of disease, the average life expectancy of an adult female mosquito is given by $\frac{1}{\mu_{V}}$, so that the average eggs laid by an adult female mosquito throughout her life span is given by

$$
\frac{\phi_{A}}{\mu_{V}}
$$

Thus, the product of (3.3) and (3.4) gives the basic offspring number of the mosquito-only population model.

The mosquito-only model (3.1) has two equilibria depending on $N_{0}$. If $N_{0} \leq 1$, then, the system (3.1) has only the trivial equilibrium called an extinction equilibrium $\left(E_{0}\right)$ given by

$$
E_{0}=(0,0)
$$

If $N_{0}>1$, then, the system (3.1) has a non-trivial equilibrium given by

$$
E_{1}=\left[K\left(1-\frac{1}{N_{0}}\right), \frac{K \sigma_{A}}{\mu_{V}}\left(1-\frac{1}{N_{0}}\right)\right] .
$$

It is worth mentioning that the trivial equilibrium $\left(E_{0}\right)$ is biologically less attractive since mosquitoes goes extinct in the population. Rewriting the mosquito-only model (3.1) in the form $\dot{x}=f(x)$, where $\Omega^{*} \subseteq \mathbb{R}_{+}^{2}$ and $f: \Omega^{*} \rightarrow \mathbb{R}_{+}^{2}$ is continuous. Then we have the following results (The proof is given in Appendix A).

Theorem 3.1. The extinction equilibrium $\left(E_{0}\right)$ is globally-asymptotically stable $(G A S)$ when $N_{0} \leq 1$ and unstable otherwise. The non-trivial equilibrium $\left(E_{1}\right)$ exists and is locally-asymptotically stable (LAS) when $N_{0}>1$.

The epidemiological implication of Theorem (3.1) is that if the basic offspring number can be brought to a value less than unity, then mosquito population will goes to extinction and the diseases dies out in time (since no horizontal transmission).

\subsection{Disease-free equilibrium}

The disease-free equilibrium (DFE) is the steady-state solution of the autonomous system (form of model (2.1)) obtained in the absence of disease. The autonomous form of (2.1) has two disease-free equilibria depending 
on the magnitude of $N_{0}$. Suppose $N_{0} \leq 1$ and the diseased compartments are zero, then the model has a mosquito extinction DFE, $E_{2}$, given by

$$
\begin{aligned}
E_{2} & =\left(S_{U}^{*}, S_{V}^{*}, W_{U}^{*}, W_{V}^{*}, E_{U}^{*}, E_{V}^{*}, I_{U}^{*}, I_{V}^{*}, R_{U}^{*}, R_{V}^{*}, A_{M}^{*}, M_{U}^{*}, M_{E}^{*}, M_{I}^{*}\right) \\
& =\left(\frac{\Pi_{H}\left(\omega_{V}+\mu_{H}\right)}{\mu_{H}\left(\omega_{V}+\mu_{H}+\xi_{V}\right)}, \frac{\Pi_{H} \xi_{V}}{\mu_{H}\left(\omega_{V}+\mu_{H}+\xi_{V}\right)}, 0,0,0,0,0,0,0,0,0,0,0,0\right) .
\end{aligned}
$$

If $N_{0}>1$, then the model has a non-mosquito extinction DFE, $E_{3}$ given by

$$
\begin{aligned}
& E_{3}= \\
& \qquad\left(\frac{\Pi_{H}\left(\omega_{V}+\mu_{H}\right)}{\mu_{H}\left(\omega_{V}+\mu_{H}+\xi_{V}\right)}, \frac{\Pi_{H} \xi_{V}}{\mu_{H}\left(\omega_{V}+\mu_{H}+\xi_{V}\right)}, 0,0,0,0,0,0,0,0, K\left(1-\frac{1}{N_{0}}\right), \frac{K \sigma_{A}}{\left(1+\alpha_{A} \epsilon_{A}\right) \mu_{V}}\left(1-\frac{1}{N_{0}}\right), 0,0\right) .
\end{aligned}
$$

Notice that $E_{2}$ is biologically less attractive (due to absence of mosquitoes), thus we concentrate on $E_{3}$. The local stability of $E_{3}$ can be established using the next generation method [39]. Let

$$
\begin{aligned}
& K_{1}=\xi_{V}+\mu_{H}, \quad K_{2}=\omega_{V}+\mu_{H}, \quad K_{3}=\sigma_{U}+\mu_{H}, \quad K_{4}=\sigma_{V}+\mu_{H}, \quad K_{5}=\gamma_{U}+\delta_{U}+\mu_{H}, \\
& K_{6}=\gamma_{V}+\delta_{V}+\mu_{H}, \quad K_{7}=\tau_{U}+\mu_{H}, \quad K_{8}=\tau_{V}+\mu_{H}, \quad K_{9}=\sigma_{A}+\mu_{A}+\mu_{A} \alpha_{L} \epsilon_{L}, \\
& K_{10}=\mu_{V}+\mu_{V} \alpha_{A} \epsilon_{A}, \quad \text { and } \quad K_{11}=\sigma_{M}+\mu_{V}+\mu_{V} \alpha_{A} \epsilon_{A} .
\end{aligned}
$$

It follows then that the vaccinated reproduction number, denoted by, $R_{0 V}$, is given by

$$
R_{0 V}=\sqrt{Q_{1}+Q_{2}}
$$

where

$$
Q_{1}=\frac{\beta_{H V}^{2} a_{M}^{2}\left(1-\epsilon_{B} \alpha_{B}\right)^{2} \sigma_{M} \sigma_{U} M_{U}^{*} S_{U}^{*}}{\left(N_{H}^{*}\right)^{2} K_{3} K_{5} K_{10} K_{11}}\left(1+\frac{\gamma_{U} \eta_{U}}{K_{7}}\right)
$$

and

$$
Q_{2}=\frac{\beta_{H V}^{2} a_{M}^{2}\left(1-\epsilon_{B} \alpha_{B}\right)^{2}\left(1-\epsilon_{V}\right) \sigma_{M} \sigma_{V} S_{V}^{*} M_{U}^{*}}{\left(N_{H}^{*}\right)^{2} K_{4} K_{6} K_{10} K_{11}}\left(\eta_{I}+\frac{\gamma_{V} \eta_{V}}{K_{8}}\right)
$$

Following Theorem 2 of [39], the following result is established.

Lemma 3.2. The DFE of the model (2.1) is locally-asymptotically stable if $R_{0 V}<1$, and unstable if $R_{0 V}>1$.

The threshold quantity $R_{0 V}$, is the vaccinated reproduction number of the disease. It represents the average number of secondary malaria cases that one infected case can generate if introduced into a population where fraction are vaccinated and the aforementioned control strategies (bed nets, adulticides and larvicides) are used. It can be interpreted as follows.

Infection in humans occurs either in the class of non-vaccinated susceptible or vaccinated susceptible. Susceptible humans acquire infections following effective contact with infectious mosquito in $M_{I}$ class. The number of new human infections in $S_{U}$ class generated by an infectious mosquito in the $M_{I}$ class is the product of the infection rate of infectious mosquito $\left(\frac{\beta_{H V} a_{M}\left(1-\epsilon_{B} \alpha_{B}\right)}{N_{H}^{*}}\right)$, probability that a mosquito survives the exposed class 
$\left(M_{E}\right)$ and move to infected class $\left(\frac{\sigma_{M}}{K_{11}}\right)$ and the average duration in the infectious stage $\left(\frac{1}{K_{10}}\right)$. Thus the average number of new human infection generated in $S_{U}$ class by an infectious mosquito (near DFE) is given by (noting that $\left.S_{U}^{*}=\Pi_{H}\left(\omega_{V}+\mu_{H}\right) /\left(\mu_{H}\left(\omega_{V}+\mu_{H}+\xi_{V}\right)\right)\right)$.

$$
\frac{\beta_{H V} a_{M}\left(1-\epsilon_{B} \alpha_{B}\right) \sigma_{M} S_{U}^{*}}{N_{H}^{*} K_{10} K_{11}}=\frac{\beta_{H V} a_{M}\left(1-\epsilon_{B} \alpha_{B}\right) \sigma_{M} \Pi_{H}\left(\omega_{V}+\mu_{H}\right)}{N_{H}^{*} K_{10} K_{11} \mu_{H}\left(\omega_{V}+\mu_{H}+\xi_{V}\right)} .
$$

Similarly, the number of infections generated by an infected mosquito in the $S_{V}$ class is the product of the infection rate of infected mosquito $\left(\frac{\beta_{H V} a_{M}\left(1-\epsilon_{B} \alpha_{B}\right)}{N_{H}^{*}}\right)$, the probability that a mosquito survives the exposed class and move to infected class $\left(\frac{\sigma_{M}}{K_{11}}\right)$ and the average duration in the infectious stage $\left(\frac{1}{K_{10}}\right)$. So that the average number of new human infection generated in $S_{V}$ class by an infectious mosquito (near DFE) is given by

$$
\frac{\beta_{H V} a_{M}\left(1-\epsilon_{B} \alpha_{B}\right) \sigma_{M}\left(1-\epsilon_{V}\right) S_{V}^{*}}{N_{H}^{*} K_{10} K_{11}}=\frac{\beta_{H V} a_{M}\left(1-\epsilon_{B} \alpha_{B}\right) \sigma_{M}\left(1-\epsilon_{V}\right) \Pi_{H} \xi_{V}}{N_{H}^{*} K_{10} K_{11} \mu_{H}\left(\omega_{V}+\mu_{H}+\xi_{V}\right)} .
$$

Susceptible mosquitoes $\left(M_{U}\right)$ acquire infection following effective contact with infectious humans (in search for blood meal) in either of $I_{U}, I_{V}, R_{U}$ or $R_{V}$ classes. The number of new mosquitoes infections generated by non-vaccinated infectious individual in $I_{U}$ class is the product of the infection rate of infectious humans $\left(\frac{\beta_{H V} a_{M}\left(1-\epsilon_{B} \alpha_{B}\right)}{N_{H}^{*}}\right)$, the probability that an individual survives the exposed class $E_{U}$ and move to infectious $I_{U}$ class $\left(\frac{\sigma_{U}}{K_{3}}\right)$ and the average time spent in $I_{U}$ class $\left(\frac{1}{K_{5}}\right)$. Furthermore, the number of new mosquito cases generated by non-vaccinated recovered humans in $R_{U}$ class (near the DFE) is the product of the infection rate of recovered humans $\left(\frac{\beta_{H V} a_{M}\left(1-\epsilon_{B} \alpha_{B}\right)}{N_{H}^{*}}\right)$, the probability that an individual survives $E_{U}$ and move to $I_{U}$ class $\left(\frac{\sigma_{U}}{K_{3}}\right)$, the probability that an individual survives $I_{U}$ and move to $R_{U}$ class $\left(\frac{\gamma_{U}}{K_{5}}\right)$ and the average duration in $R_{U}$ compartment $\left(\frac{1}{K_{7}}\right)$. Thus, the average number of new mosquitoes infection generated by non-vaccinated infectious humans (infected or recovered) is given by (noting that $M_{U}^{*}=K \sigma_{A} /\left[\mu_{V}\left(1+\alpha_{A} \epsilon_{A}\right)\right]\left(1-1 / N_{0}\right)$ ).

$$
\begin{aligned}
{\left[\frac{\beta_{H V} a_{M}\left(1-\epsilon_{B} \alpha_{B}\right) \sigma_{U}}{N_{H}^{*} K_{3} K_{5}}\right.} & \left.+\frac{\beta_{H V} a_{M}\left(1-\epsilon_{B} \alpha_{B}\right) \sigma_{U} \gamma_{U} \eta_{U}}{N_{H}^{*} K_{3} K_{5} K_{7}}\right] M_{U}^{*} \\
& =\frac{\beta_{H V} a_{M}\left(1-\epsilon_{B} \alpha_{B}\right) \sigma_{U} M_{U}^{*}}{N_{H}^{*} K_{3} K_{5}}\left(1+\frac{\gamma_{U} \eta_{U}}{K_{7}}\right) .
\end{aligned}
$$

Using similar argument as above, with $\eta_{I}$ and $\eta_{V}$ accounting for reduction in infectivity of vaccinated infectious and vaccinated recovered humans in comparison to non-vaccinated infectious humans, the average number of new mosquitoes infections caused by infectious humans in $I_{V}$ or $R_{V}$ compartments is given by

$$
\frac{\beta_{H V} a_{M}\left(1-\epsilon_{B} \alpha_{B}\right) \sigma_{V} M_{U}^{*}}{N_{H}^{*} K_{4} K_{6}}\left(\eta_{1}+\frac{\gamma_{V} \eta_{V}}{K_{8}}\right)
$$

Thus, the total number of infections generated by infectious mosquito in the non-vaccinated humans is given by the product of (3.10) and (3.12) denoted by

$$
Q_{1}=\frac{\beta_{H V}^{2} a_{M}^{2}\left(1-\epsilon_{B} \alpha_{B}\right)^{2} \sigma_{M} \sigma_{U} M_{U}^{*} S_{U}^{*}}{\left(N_{H}^{*}\right)^{2} K_{3} K_{5} K_{10} K_{11}}\left(1+\frac{\gamma_{U} \eta_{U}}{K_{7}}\right)
$$


Similarly, the total number of infections generated by infectious mosquito in the vaccinated humans is given by the product of (3.11) and (3.13) represented by

$$
Q_{2}=\frac{\beta_{H V}^{2} a_{M}^{2}\left(1-\epsilon_{B} \alpha_{B}\right)^{2}\left(1-\epsilon_{V}\right) \sigma_{M} \sigma_{V} S_{V}^{*} M_{U}^{*}}{\left(N_{H}^{*}\right)^{2} K_{4} K_{6} K_{10} K_{11}}\left(\eta_{I}+\frac{\gamma_{V} \eta_{V}}{K_{8}}\right)
$$

Thus, the square root of the sum of (3.14) and (3.15) represented by (3.8) gives the vaccinated reproduction number.

\subsection{Type reproduction number}

For a homogeneous system, the vaccinated reproduction number can be seen as the control threshold required to eliminate the disease from a community. The case is different in the case of multiple host types. The typereproduction number $(\mathbb{T})$ is defined as

$$
\mathbb{T}_{i}=e^{\mathbb{T}} K(I-(I-P) K)^{-1} e,
$$

where $I$ is an identity matrix, $P$ is a projection matrix, $e$ is a unit vector with all elements equal to zero except the $i t h$ term and $K=F V^{-1}$ is the next generation matrix with large domain. The type reproduction number correctly determines the critical control effort for heterogeneous populations [23].

We should note from the next generation matrix $\left(K=F V^{-1}\right)$ that new infections occur only in compartments $E_{U}, E_{V}$ and $M_{E}$, and therefore it can not be used to compute the type reproduction number for other infected/infectious compartments without new infection. Let the type reproduction numbers of compartments $E_{U}, E_{V}$ and $M_{E}$ be respectively denoted by $\mathbb{T}_{1}, \mathbb{T}_{2}$ and $\mathbb{T}_{3}$. From (3.16), it can be shown that

$$
\mathbb{T}_{1}=\frac{Q_{1}}{1-Q_{2}}>0, \text { so that } \mathbb{T}_{1}<1 \text {, implies } Q_{1}+Q_{2}<1 \text {, (since } Q_{1}>0 \text { and } Q_{2}>0 \text { ). }
$$

Similarly,

$$
\mathbb{T}_{2}=\frac{Q_{2}}{1-Q_{1}}>0, \text { so that } \mathbb{T}_{2}<1 \text {, implies } Q_{1}+Q_{2}<1,
$$

and,

$$
\mathbb{T}_{3}=Q_{1}+Q_{2}=R_{0 V}^{2}
$$

Hence it follows from (3.17), (3.18) and (3.19) that $\mathbb{T}_{i}<1$ (for $\left.i=1,2,3\right)$ implies $R_{0 V}<1$ (and vice versa). The quantity, $\mathbb{T}_{i}$, is the expected number of cases in compartment $i$ caused by one infected individual of type $i$ in a population where fractions are vaccinated, the infection might be directly or through chains of infections passing through individuals of other types, it singles out the required control effort when targeting the population of type $i[23]$. 


\section{ENDEMIC EQUILIBRIUM AND BACKWARD BIFURCATION}

\subsection{Endemic equilibrium}

Let the endemic equilibrium (the case when $\lambda_{H} \neq 0$ and $\lambda_{V} \neq 0$ ) of the model (2.1) be denoted by $E^{* *}=$ $\left(S_{U}^{* *}, S_{V}^{* *}, W_{U}^{* *}, W_{V}^{* *}, E_{U}^{* *}, E_{V}^{* *}, I_{U}^{* *}, I_{V}^{* *}, R_{U}^{* *}, R_{V}^{* *}\right)$, where

$$
\begin{aligned}
& S_{U}^{* *}=\frac{M_{0} \Pi_{H}}{M_{0} M_{2}-\omega_{V} \xi_{V}}, \quad S_{V}^{* *}=\frac{\Pi_{H} \xi_{V}}{M_{0} M_{2}-\omega_{V} \xi_{V}}, \quad W_{U}^{* *}=\frac{M_{0} \Pi_{H} \lambda_{H}^{* *} \sigma_{U} \gamma_{U} \tau_{U}}{\left(M_{0} M_{2}-\omega_{V} \xi_{V}\right)\left(K_{3} K_{5} K_{7} M_{1}-\lambda_{H}^{* *} M_{3}\right)}, \\
& W_{V}^{* *}=\frac{M_{0} \Pi_{H} \lambda_{H}^{* *} \sigma_{V} \gamma_{V} \tau_{V}}{\left(M_{0} M_{2}-\omega_{V} \xi_{V}\right)\left(K_{4} K_{6} K_{8} M_{1}-\lambda_{H}^{* *} M_{4}\right)}, \quad E_{U}^{* *}=\frac{\lambda_{H}^{* *}\left(S_{U}^{* *}+\left(1-\epsilon_{W}\right) W_{U}^{* *}\right)}{K_{3}}, \\
& E_{V}^{* *}=\frac{\lambda_{H}^{* *}\left[S_{V}^{* *}+\left(1-\epsilon_{W}\right) W_{V}^{* *}\right]}{K_{4}}, \quad I_{U}^{* *}=\frac{\lambda_{H}^{* *} \sigma_{U}\left[S_{U}^{* *}+\left(1-\epsilon_{W}\right) W_{U}^{* *}\right]}{K_{3} K_{5}}, \\
& I_{V}^{* *}=\frac{\lambda_{H}^{* *} \sigma_{V}\left[S_{V}^{* *}+\left(1-\epsilon_{W}\right) W_{V}^{* *}\right]}{K_{4} K_{6}}, \quad R_{U}^{* *}=\frac{\lambda_{H}^{* *} \sigma_{U} \gamma_{U}\left[S_{U}^{* *}+\left(1-\epsilon_{W}\right) W_{U}^{* *}\right]}{K_{3} K_{5} K_{7}}, \\
& R_{V}^{* *}=\frac{\lambda_{H}^{* *} \sigma_{V} \gamma_{V}\left[S_{V}^{* *}+\left(1-\epsilon_{W}\right) W_{V}^{* *}\right]}{K_{4} K_{6} K_{8}}, \quad A_{M}^{* *}=K\left[1-\frac{K_{9} K_{10}\left[\lambda_{V}^{* *}+\mu_{V}\left(1+\alpha_{A} \epsilon_{A}\right)\right]}{\phi_{V} \sigma_{A}\left[K_{10}+\lambda_{V}^{* *}\right]}\right], \\
& M_{U}^{* *}=\frac{A_{M}^{* *} \sigma_{A}}{K_{10}+\lambda_{V}^{* *}}, \quad M_{E}^{* *}=\frac{A_{M}^{* *} \lambda_{V}^{* *} \sigma_{A}}{K_{11}\left(K_{10}+\lambda_{V}^{* *}\right)}, \quad M_{I}^{* *}=\frac{A_{M}^{* *} \lambda_{V}^{* *} \sigma_{M} \sigma_{A}}{K_{10} K_{11}\left(K_{10}+\lambda_{V}^{* *}\right)},
\end{aligned}
$$

so that,

$$
\begin{aligned}
& N_{H}^{* *}=S_{U}^{* *}+S_{V}^{* *}+W_{U}^{* *}+W_{V}^{* *}+E_{U}^{* *}+E_{V}^{* *}+I_{U}^{* *}+I_{V}^{* *}+R_{U}^{* *}+R_{V}^{* *}, \quad M_{0}=\lambda_{H}^{* *}\left(1-\epsilon_{V}\right)+\omega_{V}+\mu_{H}, \\
& M_{1}=\lambda_{H}^{* *}\left(1-\epsilon_{V}\right)+\mu_{H}, \quad M_{2}=\lambda_{H}^{* *}+\xi_{V}+\mu_{H}, \quad M_{3}=\sigma_{U} \gamma_{U} \tau_{U}\left(1-\epsilon_{W}\right), \quad M_{4}=\sigma_{V} \gamma_{V} \tau_{V}\left(1-\epsilon_{W}\right),
\end{aligned}
$$

with,

$$
\lambda_{H}^{* *}(T)=\frac{\beta_{H V} M_{I}^{* *}(t)}{N_{H}^{* *}(t)}\left(1-\epsilon_{B} \alpha_{B}\right) a_{M}(T),
$$

and,

$$
\lambda_{V}^{* *}(T)=\frac{\beta_{H V}}{N_{H}^{* *}(t)}\left(1-\epsilon_{B} \alpha_{B}\right) a_{M}(T)\left[I_{U}^{* *}(t)+\eta_{I} I_{V}^{* *}(t)+\eta_{U} R_{U}^{* *}+\eta_{V} R_{V}^{* *}\right] .
$$

\subsection{Backward bifurcation}

Here we apply the method described in $[9,39]$ which is based on the use of Centre Manifold Theory to prove the existence of backward bifurcation for the autonomous version of model (2.1). We claim the following result (The proof is given in Appendix B):

Theorem 4.1. The autonomous malaria model (2.1) undergoes backward bifurcation at $R_{0 V}=1$ whenever the bifurcation coefficient given by $\boldsymbol{a}$ in equation (B.6) is positive.

The epidemiological implication of the phenomenon of backward bifurcation is that the classical requirement of $R_{0 V}<1$ is, although necessary, no longer sufficient for disease elimination. 


\subsection{Non-existence of backward bifurcation}

It is well known that disease induced death rate and imperfect vaccination are some of the major causes of backward bifurcation in vector borne disease models. Let consider the case where disease induced mortality is negligible $\left(\delta_{U}=\delta_{V}=0\right)$ and the vaccine is perfect $\left(\omega_{V}=0\right)$, then the bifurcation coefficient given by $\mathbf{a}$ in equation (B.6) reduces to

$$
\begin{aligned}
\mathbf{a}= & \sum_{k, i, j=1}^{n} v_{k} w_{I} w_{j} \frac{\partial^{2} f_{k}}{\partial x_{I} \partial x_{j}}(0,0)=-\frac{2\left(\beta_{H V}^{*}\right)^{2}\left(1-\epsilon_{B} \alpha_{B}\right)^{2} w_{14}^{2} v_{14}}{\left(N_{H}^{*}\right)^{2}}\left\{N_{H}^{*} P_{1}+P_{4} P_{9} S_{V}^{*}\left(1-\epsilon_{V}\right)+P_{2} P_{8} S_{U}^{*}\right. \\
& \left.+P_{8} P_{10}\left(N_{H}^{*} \epsilon_{W}-S_{V}^{*}\right)+P_{9} P_{11}\left(N_{H}^{*} \epsilon_{W}-S_{U}^{*}\right)+P_{9} P_{11} S_{V}^{*} \epsilon_{V}+P_{8} P_{6} S_{V}^{*}+P_{9} P_{7} S_{U}^{*}\left(1-\epsilon_{V}\right)\right\}
\end{aligned}
$$

which is less than zero provided $\epsilon_{W} \geq \max \left\{\frac{S_{V}^{*}}{N_{H}^{*}}, \frac{S_{U}^{*}}{N_{H}^{*}}\right\}$.

Lemma 4.2. The model (2.1) does not undergoes backward bifurcation at $R_{0 V}=1$ provided $\delta_{U}=\delta_{V}=\omega_{V}=0$ and $\epsilon_{W} \geq \max \left\{\frac{S_{V}^{*}}{N_{H}^{*}}, \frac{S_{U}^{*}}{N_{H}^{*}}\right\}$.

\section{AnAlysis of The NON-AUtONOMOUS MODEL}

Consider the non-autonomous model given by (2.1) with $T(t)=T_{0}\left[1+T_{1} \cos \left(\frac{2 \pi}{365}(\omega t+\phi)\right)\right]$ as defined in (2.2) and the time dependent basic offspring number given by

$$
N_{0}(t)=\frac{\phi_{A}(T) \sigma_{A}(T)\left(1-\epsilon_{L} \alpha_{L}\right)}{\left(\sigma_{A}(T)+\epsilon_{L} \alpha_{L}+\mu_{A}(T)\right)\left(1+\epsilon_{A} \alpha_{A}\right) \mu_{V}(T)}
$$

is strictly greater than 1 . To find the disease-free state of the system given by $(2.1)$, we let $E_{U}(t)=E_{V}(t)=$ $I_{U}(t)=I_{V}(t)=R_{U}(t)=R_{V}(t)=M_{E}(t)=M_{I}(t)=0$ and obtained a non-trivial disease-free state given by

$$
\begin{aligned}
E_{4} & =\left(S_{U}^{* *}, S_{V}^{* *}, W_{U}^{* *}, W_{V}^{* *}, E_{U}^{* *}, E_{V}^{* *}, I_{U}^{* *}, I_{V}^{* *}, R_{U}^{* *}, R_{V}^{* *}, A_{M}^{* *}, M_{U}^{* *}, M_{E}^{* *}, M_{I}^{* *}\right) \\
& =\left(\frac{\Pi_{H}\left(\omega_{V}+\mu_{H}\right)}{\mu_{H}\left(\omega_{V}+\mu_{H}+\xi_{V}\right)}, \frac{\Pi_{H} \xi_{V}}{\mu_{H}\left(\omega_{V}+\mu_{H}+\xi_{V}\right)}, 0,0,0,0,0,0,0,0, A_{M}^{* *}, M_{U}^{* *}, 0,0\right)
\end{aligned}
$$

where the pair $\left(A_{M}^{* *}, M_{U}^{* *}\right)$ is the unique positive $\omega$-periodic solution of

$$
\begin{aligned}
& \frac{d A_{M}^{* *}(t)}{\mathrm{d} t}=\phi_{A}(T)\left(1-\frac{A_{M}^{* *}(t)}{K}\right) N_{V}^{* *}(t)-\sigma_{A}(T) A_{M}^{* *}(t)-\left(1+\alpha_{L} \epsilon_{L}\right) \mu_{A}(T) A_{M}^{* *}(t) \\
& \frac{d M_{U}^{* *}(t)}{\mathrm{d} t}=\sigma_{A}(T) A_{M}^{* *}(t)-\left(1+\alpha_{A} \epsilon_{A}\right) \mu_{V}(T) M_{U}^{* *}(t)
\end{aligned}
$$

which is obtained when $N_{0}(t)>1$. On the other hand, a unique positive trivial non-periodic solution is obtained when $N_{0}(t) \leq 1$.

\subsection{Basic reproduction ratio}

The local-asymptotic stability of the positive periodic disease-free state $\left(E_{4}\right)$ can be established using a threshold parameter called the basic reproduction ratio [40]. We computed the basic reproduction ratio for the 
model (2.1) using the theory developed in [40] and used for many periodic models such as [1, 2, 25, 28, 31, 43] and some of the references therein.

Consider the disease compartments of the model (2.1) given by (where $T(t)$ is as defined in $(2.2)$ )

$$
\begin{aligned}
& \frac{\mathrm{d} E_{U}(t)}{\mathrm{d} t}=\lambda_{H}(T) S_{U}(t)+\lambda_{H}(T)\left(1-\epsilon_{W}\right) W_{U}(t)-\sigma_{U} E_{U}(t)-\mu_{H} E_{U}(t), \\
& \frac{\mathrm{d} E_{V}(t)}{\mathrm{d} t}=\lambda_{H}(T)\left(1-\epsilon_{V}\right) S_{V}(t)+\lambda_{H}(T)\left(1-\epsilon_{W}\right) W_{V}(t)-\sigma_{V} E_{V}(t)-\mu_{H} E_{V}(t), \\
& \frac{\mathrm{d} I_{U}(t)}{\mathrm{d} t}=\sigma_{U} E_{U}(t)-\gamma_{U} I_{U}(t)-\delta_{U} I_{U}(t)-\mu_{H} I_{U}(t), \\
& \frac{\mathrm{d} I_{V}(t)}{\mathrm{d} t}=\sigma_{V} E_{V}(t)-\gamma_{V} I_{V}(t)-\delta_{V} I_{V}(t)-\mu_{H} I_{V}(t), \\
& \frac{\mathrm{d} R_{U}(t)}{\mathrm{d} t}=\gamma_{U} I_{U}-\tau_{U} R_{U}-\mu_{H} R_{U} \\
& \frac{\mathrm{d} R_{V}(t)}{\mathrm{d} t}=\gamma_{V} I_{V}-\tau_{V} R_{V}-\mu_{H} R_{V}, \\
& \frac{\mathrm{d} M_{E}(t)}{\mathrm{d} t}=\lambda_{V}(T) M_{U}(t)-\sigma_{M}(T) M_{E}(t)-\left(1+\alpha_{A} \epsilon_{A}\right) \mu_{V}(T) M_{E}(t), \\
& \frac{\mathrm{d} M_{I}(t)}{\mathrm{d} t}=\sigma_{M}(T) M_{E}(t)-\left(1+\alpha_{A} \epsilon_{A}\right) \mu_{V}(T) M_{I}(t),
\end{aligned}
$$

where $\lambda_{H}(T)$ and $\lambda_{V}(T)$ are as defined earlier. The matrix of new infection terms $F(t)$ and that of transfer in and out of infectious compartments $V(t)$ are respectively given by

$$
F(t)=\left(\begin{array}{cccccccc}
0 & 0 & 0 & 0 & 0 & 0 & 0 & \frac{S_{H}^{*} G_{0} a_{M}(T)}{N_{H}^{*}} \\
0 & 0 & 0 & 0 & 0 & 0 & 0 & \frac{S_{V}^{*} G_{0} a_{M}(T)\left(1-\epsilon_{V}\right)}{N_{H}^{*}} \\
0 & 0 & 0 & 0 & 0 & 0 & 0 & 0 \\
0 & 0 & 0 & 0 & 0 & 0 & 0 & 0 \\
0 & 0 & 0 & 0 & 0 & 0 & 0 & 0 \\
0 & 0 & 0 & 0 & 0 & 0 & 0 & 0 \\
0 & 0 & \frac{M_{U}^{*} G_{0} a_{M}(T)}{N_{H}^{*}} & \frac{G_{0} a_{M}(T) \eta_{I} M_{U}^{*}}{N_{H}^{*}} & \frac{G_{0} a_{M}(T) \eta_{U} M_{U}^{*}}{N_{H}^{*}} & \frac{G_{0} a_{M}(T) \eta_{V} M_{U}^{*}}{N_{H}^{*}} & 0 & 0 \\
0 & 0 & 0 & 0 & 0 & 0 & 0 & 0
\end{array}\right),
$$

and

$$
V(t)=\left(\begin{array}{cccccccc}
K_{3} & 0 & 0 & 0 & 0 & 0 & 0 & 0 \\
0 & K_{4} & 0 & 0 & 0 & 0 & 0 & 0 \\
-\sigma_{U} & 0 & K_{5} & 0 & 0 & 0 & 0 & 0 \\
0 & -\sigma_{V} & 0 & K_{6} & 0 & 0 & 0 & 0 \\
0 & 0 & -\gamma_{U} & 0 & K_{7} & 0 & 0 & 0 \\
0 & 0 & 0 & -\gamma_{V} & 0 & K_{8} & 0 & 0 \\
0 & 0 & 0 & 0 & 0 & 0 & \sigma_{M}(T)+\mu_{V}(T)\left(1+\alpha_{A} \epsilon_{A}\right) & 0 \\
0 & 0 & 0 & 0 & 0 & 0 & -\sigma_{M} & \mu_{V}(T)\left(1+\alpha_{A} \epsilon_{A}\right)
\end{array}\right)
$$

where,

$$
G_{0}=\beta_{H V}\left(1-\epsilon_{B} \alpha_{B}\right) .
$$


Noticed that $F(t)$ is non-negative and $-V(t)$ is cooperative. Let $K(t)=\left(E_{U}(t), E_{V}(t), I_{U}(t), I_{V}(t)\right.$, $\left.R_{U}(t), R_{V}(t), M_{E}(t), M_{I}(t)\right)^{T}$, then the linearization of (5.2) can be re-written in the form

$$
\frac{\mathrm{d} K}{\mathrm{~d} t}=(F(t)-V(t)) K(t)
$$

Following the approach of $[25,40]$, let $Y(t, s)$ and $\Phi_{T}=Y(t, 0)$ respectively be the evolution operator and monodromy matrix of the linear $\omega$-periodic system $\frac{d y}{\mathrm{~d} t}=-V y(t), t \geq s$, that is for each $s \in \mathbb{R}$, the $8 \times 8$ matrix $Y(t, s)$ satisfies

$$
\frac{\mathrm{d} Y}{\mathrm{~d} t}=-V Y(t, s), \quad Y(s, s)=I, \quad t \geq s,
$$

where $I$ is the identity matrix of order 8 . Let $Z_{T}$ be the Banach space of all $\omega$-periodic functions equipped with the maximum norm and an $\omega$-periodic function of $s$ denoted by $\alpha(s)$ be the initial distribution of infectious individuals in the community, then the rate at which new infections are produced by an infected individual in the community who were introduced at time $s$ is given by $F(s) \alpha(s)[2,25,40]$. Likewise the distribution of new infected individuals from infections at time $s$ and remain in the infected compartments at a later time $t$ is $Y(t, s) F(s) \alpha(s)$. Therefore

$$
\theta(t)=\int_{-\infty}^{t} Y(t, s) F(s) \alpha(s) \mathrm{d} s=\int_{0}^{\infty} Y(t, t-a) F(t-a) \alpha(t-a) d a
$$

gives the cumulative distribution of new infections at time $t$ that are produced by all infected individuals $(\alpha(s))$ introduced at sometimes before $t$.

Define the linear operator $\mathcal{L}: Z_{T} \longrightarrow Z_{T}$ by

$$
(\mathcal{L} \alpha)(s)=\int_{0}^{\infty} Y(t, t-a) F(t-a) \alpha(t-a) d a \quad \forall t \in \mathbb{R}, \quad \alpha \in Z_{T} .
$$

Suppose $\rho(\mathcal{L})$ is the spectral radius of $\mathcal{L}$, then the basic reproduction ratio $\left(R_{0 T}\right)$ is given by $\rho(\mathcal{L})[40]$. It is easy to show that, in addition to Assumptions A1 to A5 of [40] satisfied by the autonomous system, the non-autonomous model (2.1) can be shown to satisfy the additional Assumptions A6 and A7 of [40]. Thus the following stability result is obtained.

Theorem 5.1. The disease free state $\left(E_{4}\right)$, of the non-autonomous model (2.1) is $L A S$ if $R_{0 T}<1$ and unstable if $R_{0 T}>1$ provided $N_{0}(t)>1$.

\section{EFFECT OF CONTROL STRATEGIES}

In this study we consider five main control strategies, namely:

(I) Bed nets-only strategy;

(II) Vaccination-only strategy;

(III) Mosquito control-only (larvicides and adulticides) strategy;

(IV) Bed nets and vaccination strategy;

(V) Bed nets, vaccination and mosquito control strategy (Hybrid strategy).

Using the functional definitions of $a_{M}, \phi_{A}, \sigma_{A}, \mu_{A}, \sigma_{M}$ and $\mu_{V}$ together with the following parameter values: $\Pi_{H}=450, K=150000, \beta_{H V}=0.64, \mu_{H}=0.0000342, \epsilon_{W}=0.5, \tau_{U}=0.1 \tau_{V}=0.02, \gamma_{U}=0.015, \gamma_{V}=0.017$, $\sigma_{U}=0.08, \sigma_{V}=0.07, \eta_{I}=0.7, \eta_{U}=0.3, \eta_{V}=0.1$ as given in Table 2 (unless otherwise stated), the model will be simulated to assess the effectiveness of these strategies (implemented singly or in combination). Since we are 




FiguRE 6. Simulation of the model (2.1) showing the total number of infected humans $\left(I_{U}+I_{V}\right)$ with different initial conditions approaching the disease free equilibrium when $R_{0 V}<1$.

interested in exploring the feasibility of disease elimination, these simulations are carried out for the special case of the model where backward bifurcation does not occur. In order to analyse the effect of the aforementioned control strategies in the presence of temperature changes, three different sets of numerical simulations are carried out, that is the cases where temperature $(T)$ is $20^{\circ} \mathrm{C}, 25^{\circ} \mathrm{C}$ and $30^{\circ} \mathrm{C}$.

\subsection{Bed nets-only strategy}

Here, the effect of using bed nets-only is assessed, by setting all other parameters related to vaccination and mosquito control to zero, that is the case when $\xi_{V}=\epsilon_{V}=\alpha_{A}=\epsilon_{A}=\alpha_{L}=\epsilon_{L}=0$. The model is simulated using the following levels of bed nets effectiveness (with bed nets efficacy of 0.5 ):

(i) Low bed nets effectiveness: $\alpha_{B}=0.1$ (i.e., only $10 \%$ of individuals uses bed nets effectively);

(ii) Moderate bed nets effectiveness: $\alpha_{B}=0.3$ (i.e., only $30 \%$ of individuals uses bed nets effectively);

(iii) High bed nets effectiveness: $\alpha_{B}=0.5$ (i.e., only $50 \%$ of individuals uses bed nets effectively).

As expected, an increase in the rate of bed net use leads to a decrease in the number of malaria cases. For instance, the resulting number of infected individuals corresponding to the low, moderate and high bed nets use (for the case when the temperature is taken to be $20^{\circ} \mathrm{C}$ ) is $18,000,12,200$ and 6,100 , respectively (Tab 3). Figure 8 shows the simulations of the model using different temperature levels and bed nets use, from which it is evident that the use of bed nets is more effective when temperature is $20^{\circ} \mathrm{C}$ (as in Fig. $8 \mathrm{~A}$ ), where the total number of infected humans is lower and approach the DFE faster. The DFE is reached by the total infected humans when $T=30^{\circ} \mathrm{C}$ (as shown in Fig. 9C) at almost half the time taken to reach the DFE when $T=25^{\circ} \mathrm{C}$ (where the total infected humans are at their peak when $T=25^{\circ} \mathrm{C}$; Fig. 8B). Figure 9D depicts the cumulative number of new cases in humans with low, medium and high rates of applying bed nets when $T=20^{\circ} \mathrm{C}$.

A contour plot of the reproduction number $R_{0 V}$ (with $\delta_{U}=\delta_{V}=\omega_{V}=0$, so that backward bifurcation does not exist) as a function of rate of bed nets applications $\left(\alpha_{B}\right)$ and the vaccine efficacy $\left(\epsilon_{B}\right)$ is depicted in Figure 23. As expected, the plot show decrease in $R_{0 V}$ values with increasing values of $\alpha_{B}$ and $\epsilon_{B}$. Furthermore, based on the parameter values used in the simulation as given in Table 2, a high use of bed nets and highly effective bed nets are required to reduce the associated reproduction number to a value below unity (at least $60 \%$ each). 
TABLE 1. Description of variables and parameters of the model (2.1).

\begin{tabular}{|c|c|}
\hline Variable & Interpretation \\
\hline$S_{U}(t)$ & Population of non-vaccinated wholly susceptible humans \\
\hline$S_{V}(t)$ & Population of vaccinated wholly susceptible humans \\
\hline$W_{U}(t)$ & Population of non-vaccinated partially immune susceptible humans \\
\hline$W_{V}(t)$ & Population of vaccinated partially immune susceptible humans \\
\hline$E_{U}(t)$ & Population of non-vaccinated exposed humans \\
\hline$E_{V}(t)$ & Population of vaccinated exposed humans \\
\hline$I_{U}(t)$ & Population of non-vaccinated infected humans \\
\hline$I_{V}(t)$ & Population of vaccinated infected humans \\
\hline$R_{U}(t)$ & Population of non-vaccinated recovered humans \\
\hline$R_{V}(t)$ & Population of vaccinated recovered humans \\
\hline$N_{H}(t)$ & Total human population \\
\hline$A_{M}(t)$ & Population of aquatic mosquitoes \\
\hline$M_{U}(t)$ & Population of susceptible adult female mosquitoes \\
\hline$M_{E}(t)$ & Population of exposed adult female mosquitoes \\
\hline$M_{I}(t)$ & Population of infected adult female mosquitoes \\
\hline$N_{V}(t)$ & Total population of adult mosquitoes \\
\hline$N_{M}(t)$ & Total population of adult and aquatic mosquitoes \\
\hline Parameter & Interpretation \\
\hline$\Pi_{H}$ & Recruitment rate of humans \\
\hline$\mu_{H}$ & Natural death rate of humans \\
\hline$\xi_{V}$ & Rate of vaccination \\
\hline$\epsilon_{V}$ & Efficacy of vaccination \\
\hline$\omega_{V}$ & Waning rate of vaccine \\
\hline$\alpha_{B}$ & Rate of using treated bed nets \\
\hline$\epsilon_{B}$ & Efficacy of bed nets \\
\hline$\epsilon_{W}$ & Modification parameter for reduction in infectivity due to prior infection \\
\hline$\sigma_{U}, \sigma_{V}$ & Progression rates \\
\hline$\gamma_{V}$ & Recovery rate of vaccinated infectious individuals \\
\hline$\gamma_{U}$ & Recovery rate of non-vaccinated infectious individuals \\
\hline$\delta_{V}$ & Disease induced death rate of vaccinated infectious individuals \\
\hline$\delta_{U}$ & Disease induced death rate of non-vaccinated infectious individuals \\
\hline$\tau_{V}$ & Rate of loosing partial immunity by recovered vaccinated individuals \\
\hline$\tau_{U}$ & Rate of loosing partial immunity by recovered non-vaccinated individuals \\
\hline$\eta_{I}$ & Modification parameter for reduction in infectiousness of individuals in $I_{V}$ class \\
\hline$\eta_{V}$ & Modification parameter for reduction in infectiousness of individuals in $R_{V}$ class \\
\hline$\eta_{U}$ & Modification parameter for reduction in infectiousness of individuals in $R_{U}$ class \\
\hline$K$ & Carrying capacity of aquatic mosquitoes \\
\hline$\alpha_{L}, \alpha_{A}$ & Rate of applying larvicides and adulticides \\
\hline$\epsilon_{L}, \epsilon_{A}$ & Efficacy of larvicides and adulticides \\
\hline$\beta_{V H}$ & Transmission probability from infectious mosquito to susceptible human \\
\hline$\beta_{H V}$ & Transmission probability from infectious human to susceptible mosquito \\
\hline$a_{M}(T)$ & Temperature dependent biting rate of mosquitoes \\
\hline$\phi_{A}(T)$ & Temperature dependent oviposition rate of mosquitoes \\
\hline$\sigma_{A}(T)$ & Temperature dependent maturation rate of aquatic mosquitoes \\
\hline$\sigma_{M}(T)$ & Temperature dependent progression rate of mosquitoes from $M_{E}$ to $M_{I}$ \\
\hline$\mu_{A}(T)$ & Temperature dependent death rate of aquatic mosquitoes \\
\hline$\mu_{V}(T)$ & Temperature dependent death rate of non-aquatic mosquitoes \\
\hline
\end{tabular}


TABLE 2. Values and ranges for the temperature-independent parameters of the autonomous model (2.1) and two choices of parameter values for which $R_{0 V}<1$ and $R_{0 V}>1$.

\begin{tabular}{|c|c|c|c|c|}
\hline Parameter & Ranges & $R_{0 V}<1$ & $R_{0 V}<1$ & References \\
\hline$\Pi_{H}$ & $10-800$ & 100 & 30 & {$[2,18]$} \\
\hline$\omega_{V}$ & $0-1$ & 0.1 & 0.1 & Estimated \\
\hline$\xi_{V}$ & $0-1$ & 0.3 & 0.6 & {$[35]$} \\
\hline$\epsilon_{V}$ & $0-1$ & 0.3 & 0.3 & {$[35,41]$} \\
\hline$\mu_{H}$ & $0.00003-0.00006$ & 0.0000342 & 0.0000548 & {$[10,18,30]$} \\
\hline$\alpha_{B}$ & $0-1$ & 0.2 & 0.8 & [35] \\
\hline$\epsilon_{B}$ & $0-1$ & 0.5 & 0.5 & Assumed \\
\hline$\epsilon_{W}$ & $0-1$ & 0.5 & 0.5 & Assumed \\
\hline$\tau_{U}$ & $0.000055-0.011$ & 0.1 & 0.1 & {$[2,10,30]$} \\
\hline$\tau_{V}$ & $0.000055-0.011$ & 0.02 & 0.02 & {$[2,10,30]$} \\
\hline$\eta_{I}$ & $0-1$ & 0.6 & 0.7 & {$[18,31]$} \\
\hline$\eta_{U}$ & $0-1$ & 0.3 & 0.3 & {$[18,31]$} \\
\hline$\eta_{V}$ & $0-1$ & 0.1 & 0.1 & {$[18,31]$} \\
\hline$\sigma_{U}$ & $0.067-0.2$ & 0.08 & 0.08 & {$[10,25,30]$} \\
\hline$\sigma_{V}$ & $0.077-0.2$ & 0.07 & 0.07 & {$[10,25,30]$} \\
\hline$\gamma_{U}$ & $0.0014-0.017$ & 0.015 & 0.015 & {$[2,10,30]$} \\
\hline$\gamma_{V}$ & $0.0014-0.017$ & 0.016 & 0.017 & {$[2,10,30]$} \\
\hline$\delta_{U}$ & $0.0001-0.0004$ & 0.0015 & 0.004 & {$[2,10,30]$} \\
\hline$\delta_{V}$ & $0.0001-0.0003$ & 0.001 & 0.003 & {$[2,10,30]$} \\
\hline$K$ & $50-3300000$ & 10000 & 50000 & {$[2,24,36]$} \\
\hline$\phi_{A}$ & $1-500$ & 20 & 40 & {$[24,25,36]$} \\
\hline$\sigma_{A}$ & $0.02-0.27$ & 0.2 & 0.2 & {$[24,30,36]$} \\
\hline$\alpha_{L}$ & $0-1$ & 0.6 & 0.2 & Estimated \\
\hline$\alpha_{A}$ & $0-1$ & 0.625 & 0.375 & Estimated \\
\hline$\epsilon_{L}$ & $0-1$ & 0.5 & 0.5 & Estimated \\
\hline$\epsilon_{A}$ & $0-1$ & 0.8 & 0.8 & Estimated \\
\hline$\sigma_{M}$ & $0.029-0.33$ & 0.5 & 0.7 & {$[10,25,30]$} \\
\hline$\mu_{A}$ & $0.001-0.2$ & 0.1 & 0.019 & {$[10,25,30]$} \\
\hline$\mu_{V}$ & $0.04762-0.07143$ & 0.0529 & 0.04762 & {$[10,18,30]$} \\
\hline$a_{M}$ & $0.1-1$ & 0.4 & 0.6 & {$[2,10,30]$} \\
\hline$\beta_{V H}$ & $0.0027-0.64$ & 0.44 & 0.64 & {$[2,10,18]$} \\
\hline$\beta_{H V}$ & $0.072-0.64$ & 0.44 & 0.64 & {$[2,10,30]$} \\
\hline
\end{tabular}

TABLE 3. Number of infected individuals using bed nets-only strategy.

\begin{tabular}{llll}
\hline Level of & Temp at & Temp at & Temp at \\
bed net use & $20^{\circ} \mathrm{C}$ & $25^{\circ} \mathrm{C}$ & $30^{\circ} \mathrm{C}$ \\
\hline Low $\left(\alpha_{B}=0.1\right)$ & 18000 & 98000 & 80000 \\
Moderate $\left(\alpha_{B}=0.3\right)$ & 12200 & 82000 & 67000 \\
High $\left(\alpha_{B}=0.5\right)$ & 6100 & 68000 & 56000 \\
\hline
\end{tabular}

\subsection{Vaccination-only strategy}

The effect of the use of vaccination only for temperatures of $T=20^{\circ} \mathrm{C}, T=25^{\circ} \mathrm{C}$ and $T=30{ }^{\circ} \mathrm{C}$ with vaccine efficacy of 0.75 is investigated. For the vaccination-only strategy, we have $\alpha_{A}=\epsilon_{A}=\alpha_{B}=\epsilon_{B}=\alpha_{L}=\epsilon_{L}=0$. Simulation of the model (2.1) are carried out to assess the impact of vaccination in reducing the malaria burden under the following levels of vaccination effectiveness. 


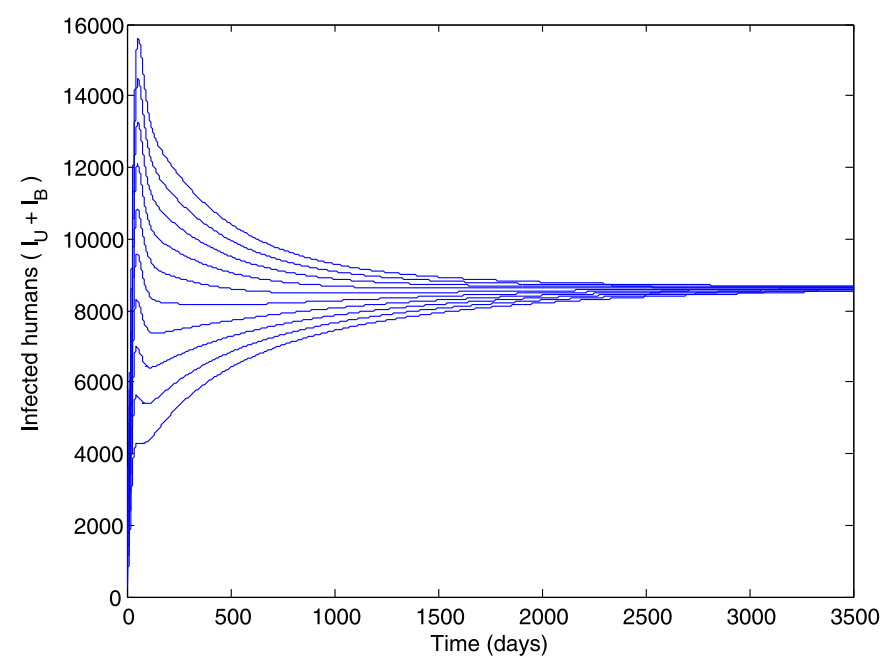

FiguRE 7. Simulation of the model (2.1) showing the total number of infected humans $\left(I_{U}+I_{V}\right)$ with different initial conditions approaching an endemic equilibrium when $R_{0 V}>1$.
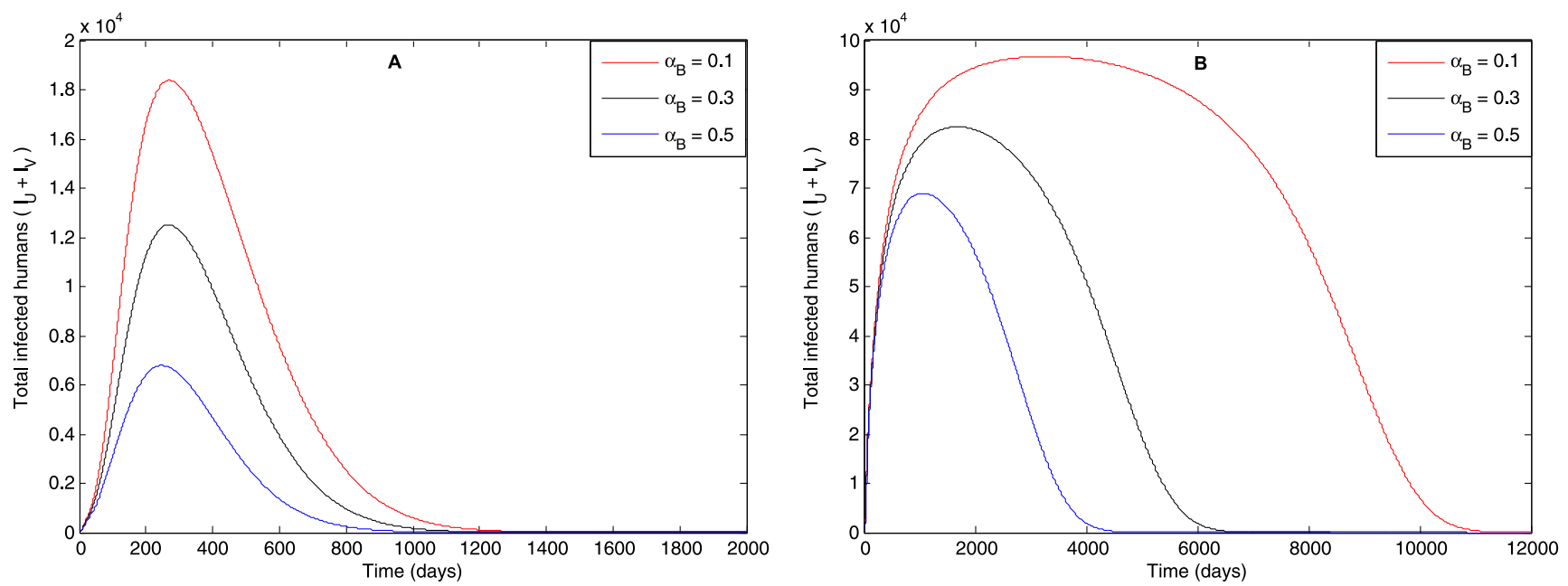

Figure 8. Simulations of the model (2.1) showing the total number of infected humans $\left(I_{U}+\right.$ $I_{V}$ ) with: (A) When $T=20$ having; $R_{0 V}=0.1233$ when $\alpha_{B}=0.1, R_{0 V}=0.1103$ when $\alpha_{B}=$ 0.3 , and $R_{0 V}=0.0973$ when $\alpha_{B}=0.5$ with $N_{0}=116.9812$. (B) When $T=25$ having; $R_{0 V}=$ 0.3492 when $\alpha_{B}=0.1, R_{0 V}=0.3125$ when $\alpha_{B}=0.3$, and $R_{0 V}=0.2757$ when $\alpha_{B}=0.5$ also with $N_{0}=116.7733$.

(i) Low vaccination effectiveness: $\xi_{V}=0.1$ (i.e., only $10 \%$ of individuals are vaccinated effectively);

(ii) Moderate vaccination effectiveness: $\xi_{V}=0.3$ (i.e., only $30 \%$ of individuals are vaccinated effectively);

(iii) High vaccination effectiveness: $\xi_{V}=0.5$ (i.e., only $50 \%$ of individuals are vaccinated effectively).

Similar to the use of bed nets, vaccination is more effective in reducing the total number of infected humans and time taken to reach the DFE when the temperature is $20^{\circ} \mathrm{C}$ (Fig. 10A), where as similar effect for both temperatures of $25^{\circ} \mathrm{C}$ (Fig. 10B) and $30^{\circ} \mathrm{C}$ (Fig. 11C) are obtained. A cumulative number of new cases in humans with low, medium and high rates of vaccine application at $T=20^{\circ} \mathrm{C}$ is depicted in Figure 11D. 

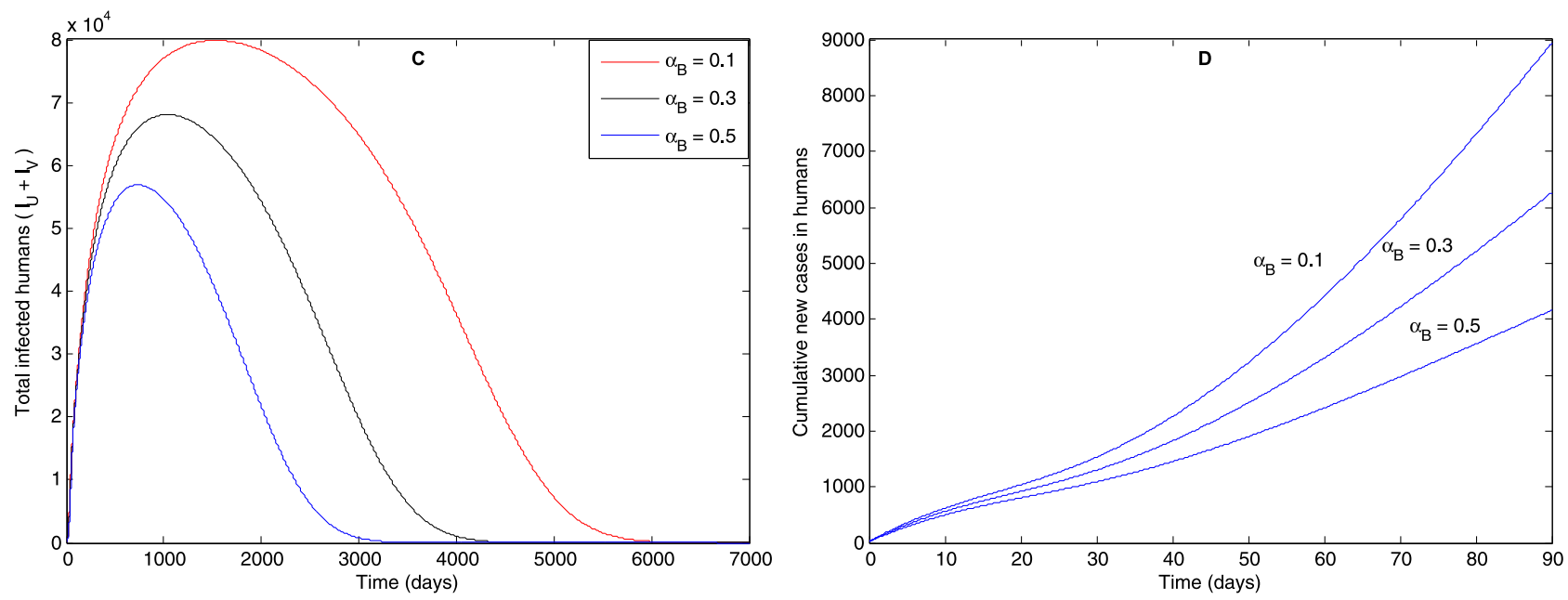

Figure 9. Simulations of the model (2.1) showing: (C) The total number of infected humans $\left(I_{U}+I_{V}\right)$ with $T=30$, showing $R_{0 V}=0.3052, N_{0}=41.4528$ when $\alpha_{B}=0.1, R_{0 V}=$ $0.2731, N_{0}=41.4528$ when $\alpha_{B}=0.3$, and $R_{0 V}=0.2410, N_{0}=41.4528$ when $\alpha_{B}=0.5$. (D) Cumulative new cases in humans at $T=20^{\circ} \mathrm{C}$ with different levels of applications.
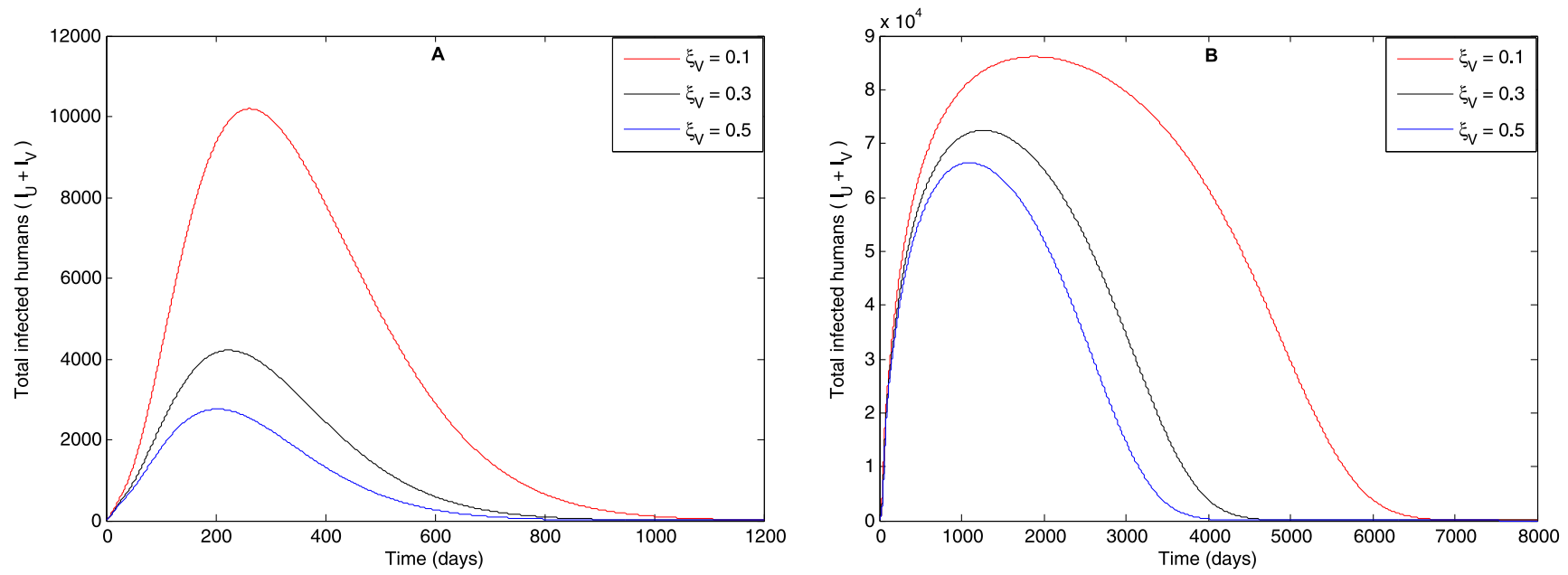

FiguRE 10. Simulations of the model (2.1) showing the total number of infected humans $\left(I_{U}+I_{V}\right)$ with: (A) $T=20$ such that $R_{0 V}=0.2838$ for $\xi_{V}=0.1, R_{0 V}=0.3353$ for $\xi_{V}=0.3$, $R_{0 V}=0.3508$ for $\xi_{V}=0.5$ and $N_{0}=116.9812$. (B) $T=25$ such that $R_{0 V}=0.8036$ for $\xi_{V}=0.1$, $R_{0 V}=0.9493$ for $\xi_{V}=0.3, R_{0 V}=0.9931$ for $\xi_{V}=0.5$ and $N_{0}=116.7733$.

As expected the vaccination reproduction number decreases with increase in the rate of application of vaccines. At $T=20^{\circ} \mathrm{C}$, total infected humans reach the DFE in less than 1,200 days, while it reaches the DFE in about 8,000 days for the case when $T=25^{\circ} \mathrm{C}$ and less than 5,000 days when $T=30^{\circ} \mathrm{C}$ as in Figures $10 \mathrm{~A}, 10 \mathrm{~B}$ and $11 \mathrm{C}$.

A contour plot of the vaccination reproduction number $R_{0 V}$ (with $\delta_{U}=\delta_{V}=\omega_{V}=0$ ) as a function of fraction of vaccinated individuals at steady-state $V_{H}^{*}=S_{V}^{*} / N_{H}^{*}$ and the vaccine efficacy $\left(\epsilon_{V}\right)$ is depicted in Figure 24 . The plot show decrease in $R_{0 V}$ values with increasing values of $V_{H}^{*}$ and $\epsilon_{V}$, and even if $90 \%$ of individuals are 

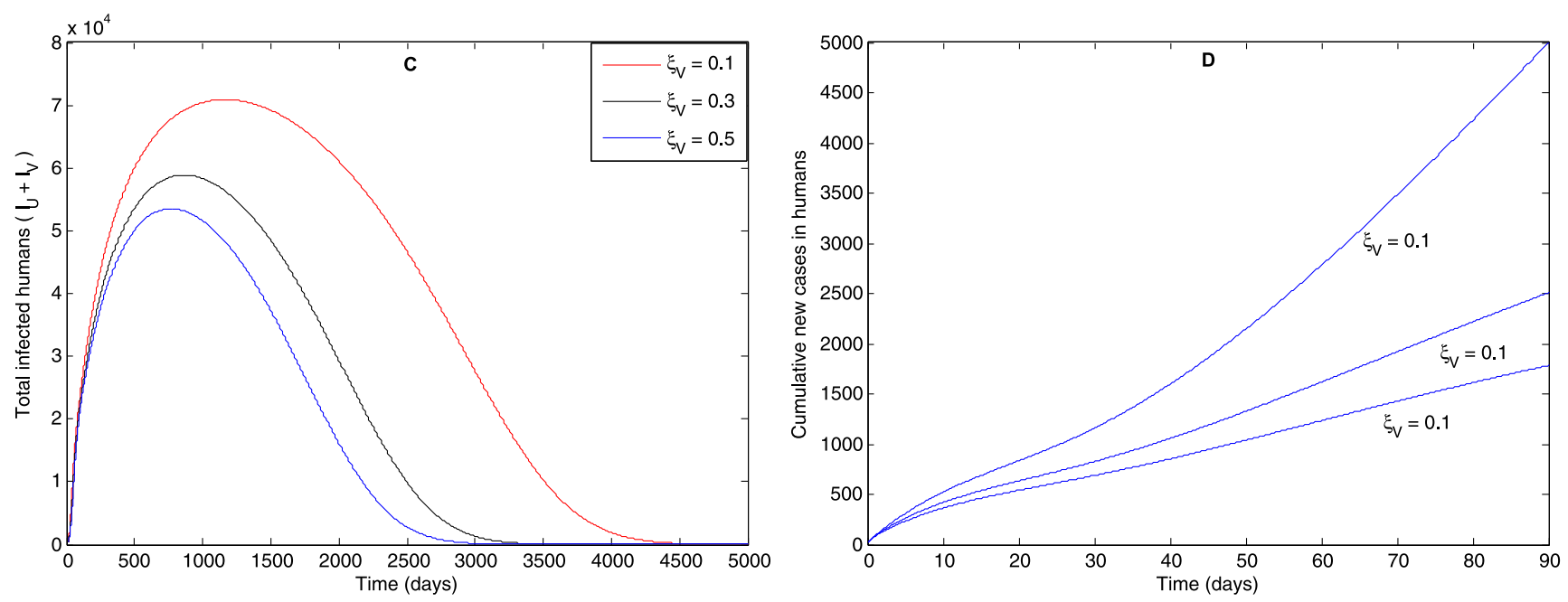

Figure 11. Simulations of the model (2.1) showing: (C) The total number of infected humans $\left(I_{U}+I_{V}\right)$ with $T=30$ which implies $R_{0 V}=0.7000$ for $\xi_{V}=0.1, R_{0 V}=0.8267$ for $\xi_{V}=0.3$, $R_{0 V}=0.8648$ for $\xi_{V}=0.5$ and $N_{0}=41.4528$. (D) Cumulative number of new human cases for $T=20$ with different levels of applications.
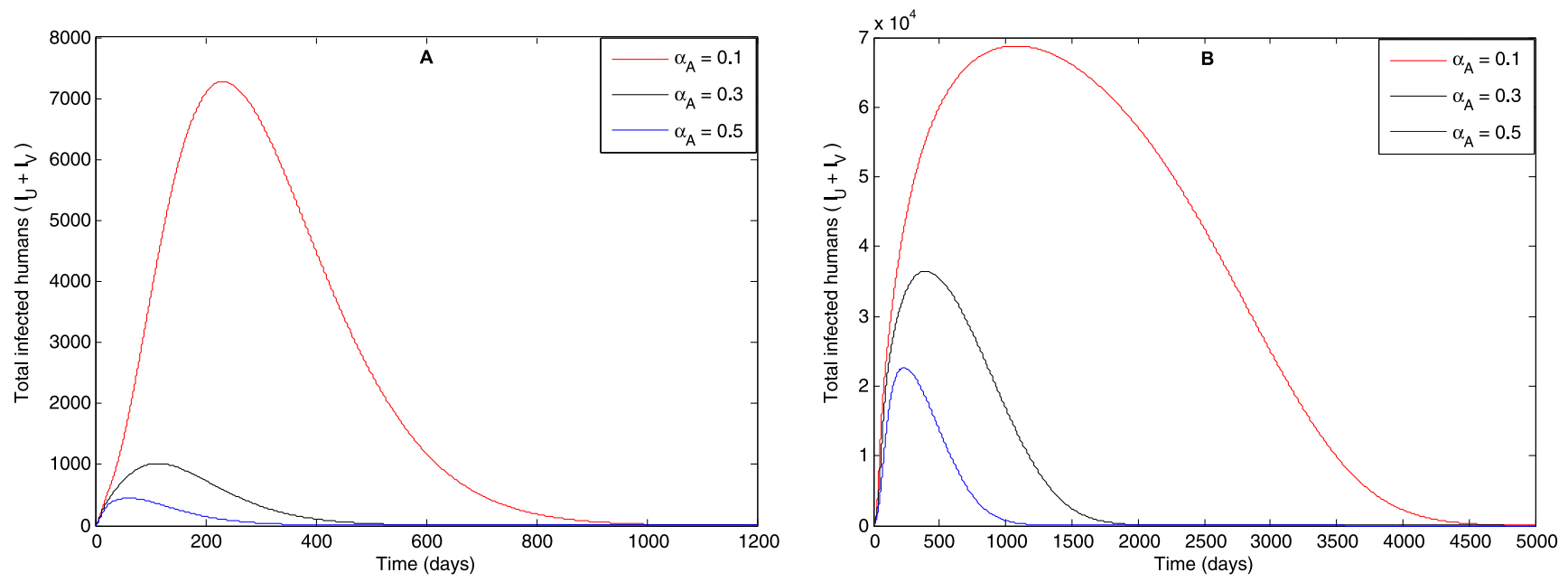

Figure 12. Simulations of the model (2.1) showing the total number of infected humans $\left(I_{U}+I_{V}\right)$ with: (A) Obtained for $T=20$, where $R_{0 V}=0.0957, N_{0}=93.1507$ for $\alpha_{A}=0.1$, $R_{0 V}=0.0600, N_{0}=66.1852$ for $\alpha_{A}=0.3$, and $R_{0 V}=0.0420, N_{0}=51.3270$ for $\alpha_{A}=0.5$. (B) Obtained for $T=25$, with $R_{0 V}=0.2786, N_{0}=93.6979$ for $\alpha_{A}=0.1, R_{0 V}=0.1810, N_{0}=$ 67.1564 for $\alpha_{A}=0.3$, and $R_{0 V}=0.1299, N_{0}=52.3324$ for $\alpha_{A}=0.5$.

vaccinated, the disease will still persist in the population, unless the vaccine efficacy is at least $50 \%$ (to reduce the vaccination reproduction number to a value below unity). 

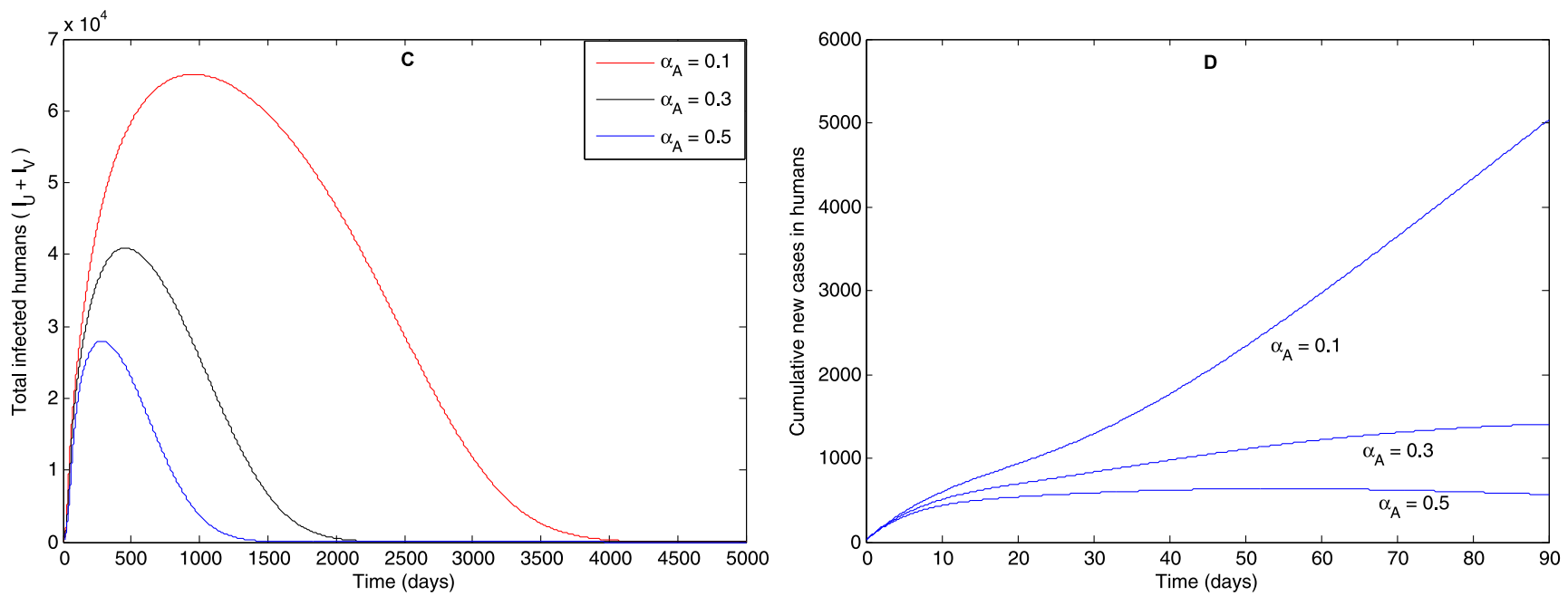

Figure 13. Simulations of the model (2.1) showing: (C) The total number of infected humans $\left(I_{U}+I_{V}\right)$ with $T=30$, where $R_{0 V}=0.2670, N_{0}=35.9381$ for $\alpha_{A}=0.1, R_{0 V}=0.1954, N_{0}=$ 28.3856 for $\alpha_{A}=0.3$, and $R_{0 V}=0.1510, N_{0}=23.4562$ for $\alpha_{A}=0.5$. (D) Cumulative number of new cases in humans with different level of interventions and $T=20^{\circ} \mathrm{C}$.


Figure 14. Simulations of the model (2.1) showing the total number of infected humans $\left(I_{U}+\right.$ $I_{V}$ ) with: (A) Obtained for $T=20$, so that $R_{0 V}=0.2696$ for $\alpha_{V}=\alpha_{B}=0.1, R_{0 V}=0.2850$ for $\alpha_{V}=\alpha_{B}=0.3$, and $R_{0 V}=0.2631$ for $\alpha_{V}=\alpha_{B}=0.5$ with $N_{0}=116.9812$. (B) Obtained for $T=25$, so that $R_{0 V}=0.7634$ for $\alpha_{V}=\alpha_{B}=0.1, R_{0 V}=0.8069$ for $\alpha_{V}=\alpha_{B}=0.3$, and $R_{0 V}=0.7448$ for $\alpha_{V}=\alpha_{B}=0.5$ with $N_{0}=116.7733$.

\subsection{Mosquito control-only strategy (adulticides strategy)}

Here, the effect of the use of adulticides only with efficacy of 0.5 is simulated, that is when $\alpha_{B}=\epsilon_{B}=\alpha_{L}=$ $\epsilon_{L}=\xi_{V}=\epsilon_{V}=0$. Notice that the use of larvicides has less or no effect in the dynamics of infected mosquitoes (it mainly affects the basic offspring number).

Further simulations were carried out to assess the impact of mosquito control-only strategy using adulticides. The following levels of adulticides effectiveness are considered: 



Figure 15. Simulations of the model (2.1) showing; (C) The total number of infected humans $\left(I_{U}+I_{V}\right)$ with $T=30$, such that $R_{0 V}=0.6650$ for $\alpha_{V}=\alpha_{B}=0.1, R_{0 V}=0.7027$ for $\alpha_{V}=$ $\alpha_{B}=0.3, R_{0 V}=0.6486$ for $\alpha_{V}=\alpha_{B}=0.5$ and $N_{0}=41.4528$. (D) Cumulative number of cases in humans with different levels of applications and $T=20^{\circ} \mathrm{C}$.


Figure 16. Simulations of the model (2.1) showing the total number of infected humans $\left(I_{U}+I_{V}\right)$ with: (A) Obtained when $T=20$, such that $R_{0 V}=0.0692, N_{0}=70.9784$ for low, $R_{0 V}=0.0309, N_{0}=34.1664$ for medium, and $R_{0 V}=0.0171, N_{0}=20.0347$ for high controls. (B) Obtained when $T=25$, such that $\nmid R_{0 V}=0.2017, N_{0}=85.1115$ for low, $R_{0 V}=$ $0.0937, N_{0}=51.5536$ for medium, and $R_{0 V}=0.0533, N_{0}=34.7858$ for high controls.

(i) Low adulticides effectiveness: $\alpha_{A}=0.1$ (i.e., only $10 \%$ applies adulticides effective);

(ii) Moderate adulticides effectiveness: $\alpha_{A}=0.3$ (i.e., only $30 \%$ applies adulticides effective);

(iii) High adulticides effectiveness: $\alpha_{A}=0.5$ (i.e., only $50 \%$ applies adulticides effective).

This control strategy shows the biggest positive effect in both reducing the total number of infected individuals and the effect of using different levels of controls for different temperatures compared to other forms of single controls. For $T=20^{\circ} \mathrm{C}$ (as shown in Fig. 12A), mosquito control measure at the rate $\alpha_{A}=0.5$ pushes the total 

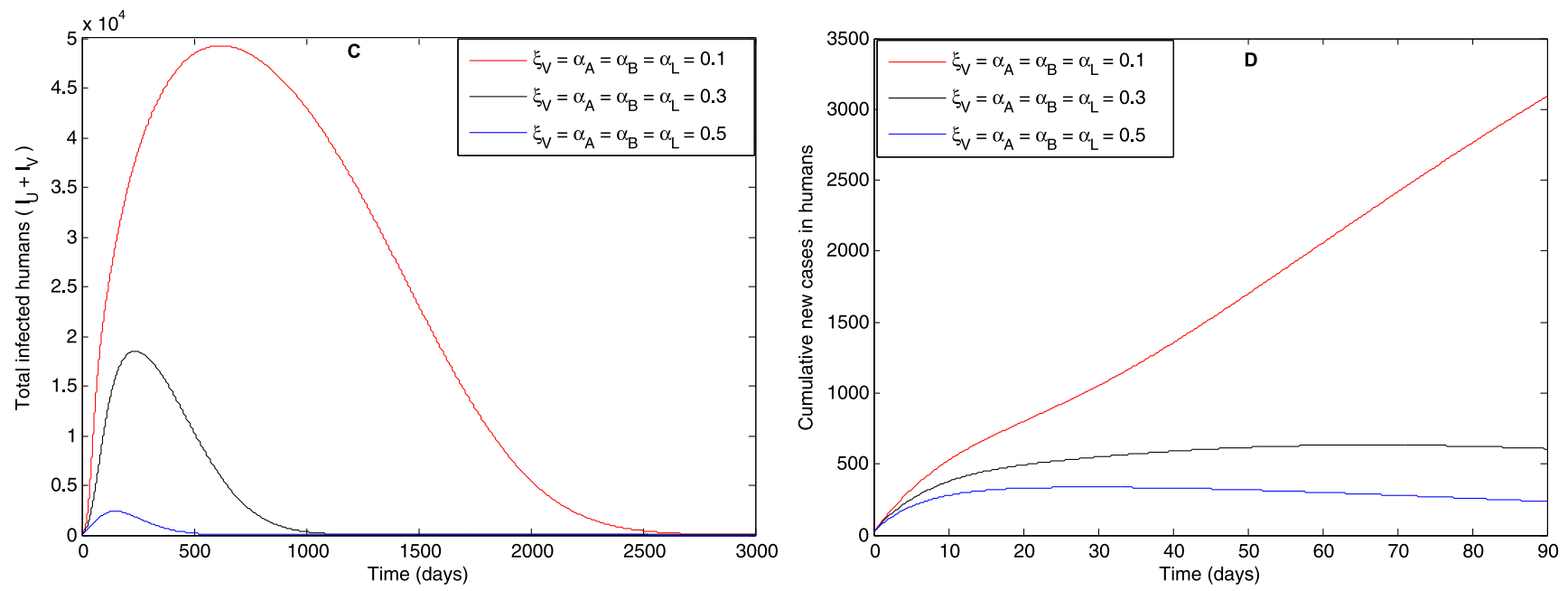

FiguRE 17. Simulations of the model (2.1) showing: (C) The total number of infected humans $\left(I_{U}+I_{V}\right)$ when $T=30$, such that $R_{0 V}=0.1932, N_{0}=34.3476$ for low, $R_{0 V}=0.1010, N_{0}=$ 24.9232 for medium, and $R_{0 V}=0.0619, N_{0}=19.0463$ for high controls. (D) Cumulative new cases in humans when $T=20$ with different levels of interventions.
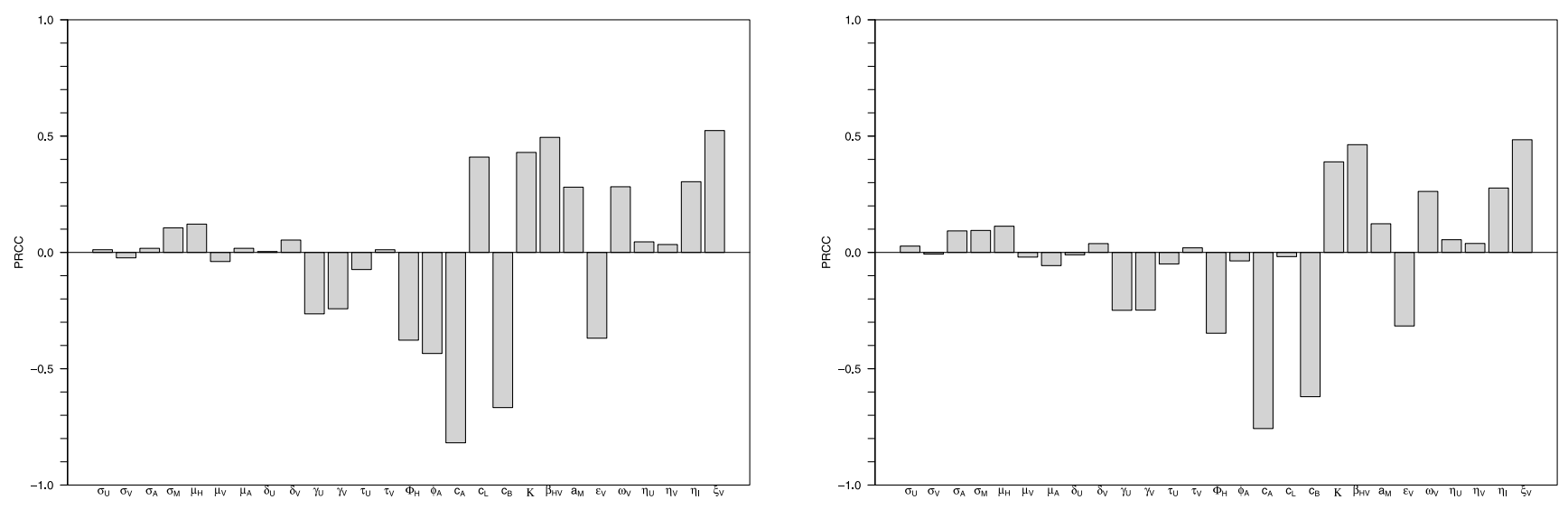

Parameters



Figure 18. Partial rank correlation coefficient (PRCC) of the model parameters with $R_{0 V}$ as the output function for temperature between $15^{\circ} \mathrm{C}-20^{\circ} \mathrm{C}$ and between $20^{\circ} \mathrm{C}-25^{\circ} \mathrm{C}$ respectively.

number of infected humans to reach the DFE at the shortest period in comparison to when $T=25^{\circ} \mathrm{C}$ (as in Fig. 12B), and when $T=30^{\circ} \mathrm{C}$ (Fig. 13C). The cumulative number of new cases in humans with low, medium and high rates of applying adulticides at $T=20^{\circ} \mathrm{C}$ is depicted in Figure 13D.

A contour plot of the reproduction number $R_{0 V}$ (with $\delta_{U}=\delta_{V}=\omega_{V}=0$ ) as a function of rate of adulticides applications $\left(\alpha_{A}\right)$ and the efficacy of adulticides $\left(\epsilon_{A}\right)$ is depicted in Figure 25. The figure show that, based on the parameter values used, a high rate of application of adulticides with high efficacy rate (at least $85 \%$ each) is required to reduce the associated reproduction number to a value below unity, so that malaria could be control from the community. 

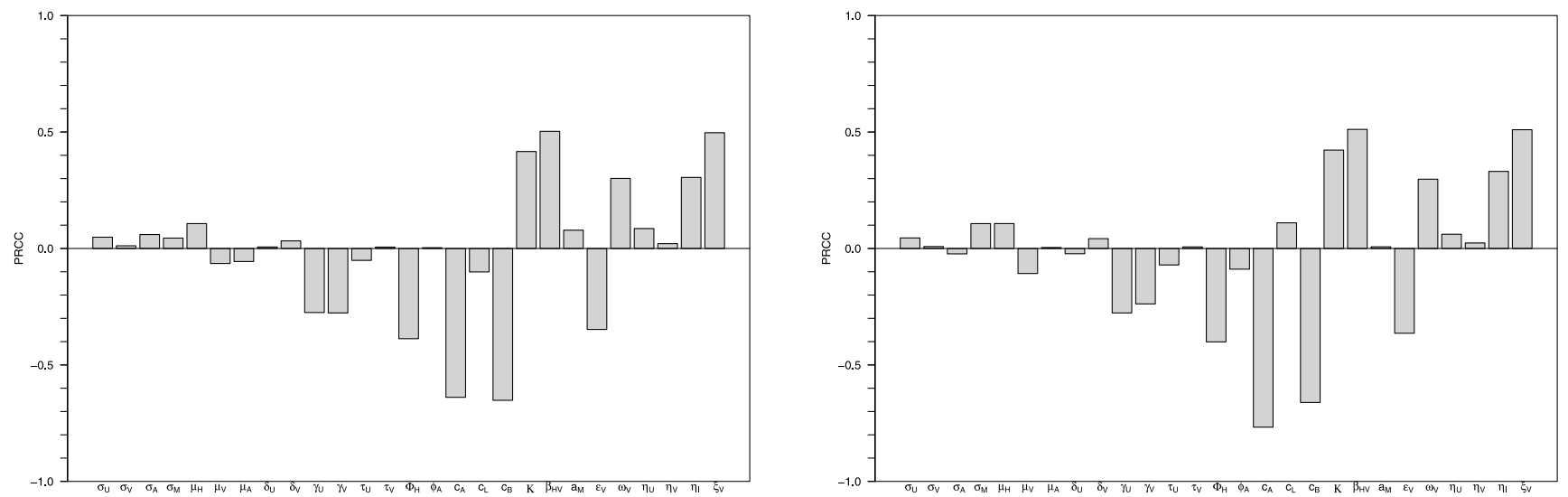

Parameters

Parameters

Figure 19. Partial rank correlation coefficient (PRCC) of the model parameters with $R_{0 V}$ as the output function for temperature between $25^{\circ} \mathrm{C}-30^{\circ} \mathrm{C}$ and $30^{\circ} \mathrm{C}-35^{\circ} \mathrm{C}$ respectively.



Parameters

Figure 20. Partial rank correlation coefficient (PRCC) of the model parameters with $R_{0 V}$ as the output function and constant temperature.

\subsection{Bed nets and vaccination strategy}

The combined effect of the use of bed nets and imperfect vaccination are explored here. Vaccine efficacy of 0.75 and bed net efficacy of 0.5 are used. For this simulation, parameters related to other control strategies are set to zero (i.e., $\epsilon_{A}=\alpha_{A}=\epsilon_{L}=\alpha_{L}=0$ ).

The model is simulated using the following levels of bed nets and vaccination effectiveness at various temperature levels $\left(20^{\circ} \mathrm{C}, 25^{\circ} \mathrm{C}\right.$ and $\left.30^{\circ} \mathrm{C}\right)$ :

(i) Low bed nets and vaccination effectiveness: $\alpha_{B}=\xi_{V}=0.1$ (i.e., only $10 \%$ of individuals uses bed nets and vaccinated effectively);

(ii) Moderate bed nets and vaccination effectiveness: $\alpha_{B}=\xi_{V}=0.3$ (i.e., only $30 \%$ of individuals uses bed nets and vaccinated effectively);

(iii) High bed nets and vaccination effectiveness: $\alpha_{B}=\xi_{V}=0.5$ (i.e., only $50 \%$ of individuals uses bed nets and vaccinated effectively). 


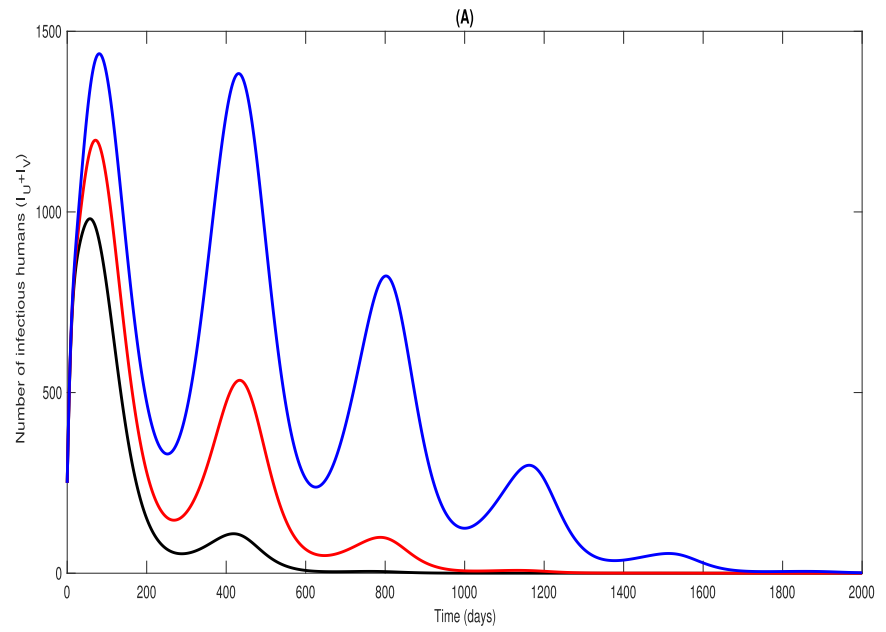

FiguRE 21. Simulation of the model (2.1) showing the total number of infected humans $\left(I_{U}+\right.$ $I_{V}$ ) with different initial conditions approaching (A) DFE for the case when $R_{0 T}<1$ and (B) an endemic equilibrium for the case when $R_{0 T}>1$. Parameter values used are as given in Table 2 with generalized temperature function $T(t)=T_{0}\left[1+T_{1} \cos \left(\frac{2 \pi}{365}(\omega t+\phi)\right)\right]$.


FiguRE 22. Simulation of the model (2.1) showing the effect of vaccination on disease incidence with seasonal variation. Parameter values used are as given in Table 2 with generalized temperature function $T(t)=T_{0}\left[1+T_{1} \cos \left(\frac{2 \pi}{365}(\omega t+\phi)\right)\right]$.

As expected, the combined use of bed nets and vaccination is more effective than the singular use of bed nets or vaccine. Figures 14 and 15 depict the simulations of the model (2.1) with the use of bed nets and vaccination, from which it is evident that the total infected humans reach the DFE (for low, medium and high application of controls) faster than the separate use of the controls for both $T=20^{\circ}$ (Fig. 14A), $T=25^{\circ} \mathrm{C}$ (Fig. 14B), and $T=30^{\circ} \mathrm{C}$ (Fig. 15C). The cumulative number of new cases in humans with low, medium and high rates of applying bed nets and vaccines at $T=20^{\circ} \mathrm{C}$ is depicted in Figure 15D.

\subsection{Hybrid strategy}

The potential impact of using all controls are examined, using the following effectiveness levels: 


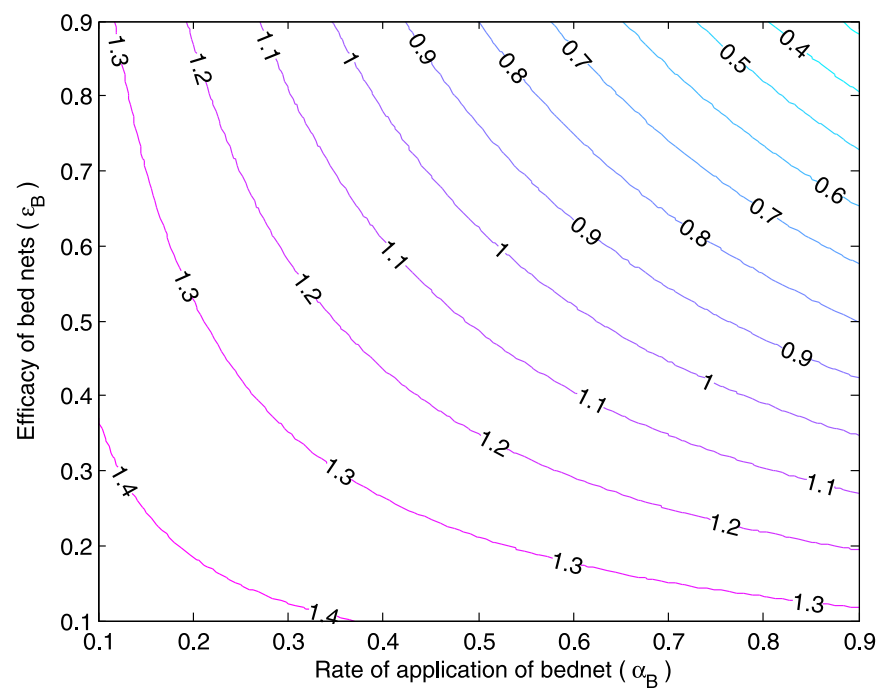

Figure 23. Simulation of the model (2.1) showing contour plot of $R_{0 V}$ as a function of bednets application $\left(\alpha_{B}\right)$ and the efficacy of bednets $\left(\epsilon_{B}\right)$. Parameter values used are as given in Table 2 with $\delta_{U}=\delta_{V}=\omega_{V}=0$.

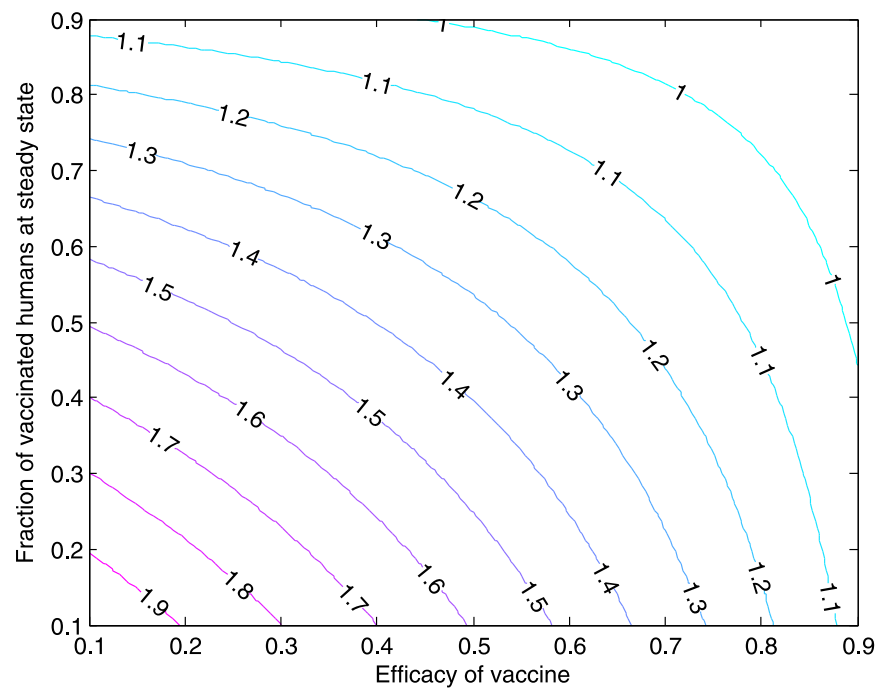

Figure 24. Simulation of the model (2.1) showing contour plot of $R_{0 V}$ as a function of fraction of vaccinated humans at steady state $\left(V_{H}^{*}=S_{V}^{*} / N_{H}^{*}\right)$ and the vaccine efficacy $\left(\epsilon_{V}\right)$. Parameter values used are as given in Table 2 with $\delta_{U}=\delta_{V}=\omega_{V}=0$.

(i) Low effectiveness: $\epsilon_{A}=\epsilon_{L}=\alpha_{B}=\xi_{V}=0.1$ (i.e., combined measures at only $10 \%$ effectiveness);

(ii) Moderate effectiveness: $\epsilon_{A}=\epsilon_{L}=\alpha_{B}=\xi_{V}=0.3$ (i.e., combined measures at only $30 \%$ effectiveness);

(iii) High effectiveness: $\epsilon_{A}=\epsilon_{L}=\alpha_{B}=\xi_{V}=0.5$ (i.e., combined measures at only $50 \%$ effectiveness).

For high levels of intervention, the total number of infected humans is lower and reach the DFE at a very short time for the different temperature levels used. For instance, the total infected humans reach the DFE in less than 1,000 days when $T=20^{\circ} \mathrm{C}$ as depicted in (Fig. 16A) and it reaches the DFE in about 3,000 days when $T=25^{\circ} \mathrm{C}$ (Fig. 16B) and $T=30^{\circ} \mathrm{C}$ (Fig. 17C). The cumulative number of new cases in humans with 




FIgURE 25. Simulation of the model (2.1) showing contour plot of $R_{0 V}$ as a function of adulticides application $\left(\alpha_{A}\right)$ and the efficacy of bednets $\left(\epsilon_{A}\right)$. Parameter values used are as given in Table 2 with $\delta_{U}=\delta_{V}=\omega_{V}=0$.

low, medium and high rates of applying bed nets, vaccines and adulticides at $T=20^{\circ} \mathrm{C}$ is depicted in Figure $17 \mathrm{D}$.

\section{SEnsitivity ANALYSis AND NUMERICAL SIMULATION}

In this section, the partial rank correlation coefficient (PRCC) of the parameters of the vaccinated reproduction number for different temperature ranges are given. In addition, numerical simulations of the model are also presented.

\subsection{Sensitivity analysis}

The partial rank correlation coefficient is a sampling based sensitivity index that measures the strength of the linear associations between a dependent variable (in this case the vaccinated reproduction number), and independent variables (its parameters) after removing the linear effect of other parameter values. We consider the cases for constant and various temperatures. Using the vaccinated reproduction number as the output for temperature values for ranges $15{ }^{\circ} \mathrm{C}-20^{\circ} \mathrm{C}, 20^{\circ} \mathrm{C}-25^{\circ} \mathrm{C}, 25^{\circ} \mathrm{C}-30^{\circ} \mathrm{C}, 30^{\circ} \mathrm{C}-35^{\circ} \mathrm{C}$ and that of constant parameter values with a confidence interval of $95 \%$ and 1000 number of boots, the PRCC are obtained.

Tables $4-8$ show the PRCC values, bias, standard error, minimum and maximum confidence interval for each of the 27 parameters of the basic reproduction ratio with the aforementioned temperature intervals and constant temperature, respectively. Similarly, Figures 18, 19 and 20 show the bar plot of the PRCC of the parameters of the basic reproduction ratio as temperature varies and constant temperature. For the different temperature ranges, the rate of vaccination $\xi_{V}$, efficacy of vaccination $\epsilon_{V}$ and rate of successful use of bed nets $C_{B}$ show little variation as temperature varies. The rate of vaccination is positively correlated to $R_{0 V}$ due to the imperfect vaccine (not $100 \%$ effective), thus vaccinated individuals can still acquire infections.

The use of larvicides is positively correlated to $R_{0 V}$ for temperature ranging from $15{ }^{\circ} \mathrm{C}$ to $20^{\circ} \mathrm{C}$ with PRCC value of +0.4099 , but when the temperature range is from $20^{\circ} \mathrm{C}$ to $25^{\circ} \mathrm{C}$, the PRCC becomes negatively correlated to $R_{0 V}$ with value of -0.0179 , it remains negative with PRCC value of -0.1008 when the temperature is between $25^{\circ} \mathrm{C}-30^{\circ} \mathrm{C}$ and returns to positive for temperature of $30^{\circ} \mathrm{C}-35^{\circ} \mathrm{C}$ having PRCC of +0.1099 , where as 
TABLE 4. PRCC for parameters of the basic reproduction ratio with temperature of $15{ }^{\circ} \mathrm{C}-20^{\circ} \mathrm{C}$.

\begin{tabular}{|c|c|c|c|c|c|}
\hline Parameters & PRCC & Bias & Std. error & Min. c.i. & Max. c.i. \\
\hline$\sigma_{U}$ & +0.0116573 & -0.0007059 & 0.0327846 & -0.0507698 & +0.0733284 \\
\hline$\sigma_{V}$ & -0.0232599 & -0.0011866 & 0.0319199 & -0.0859427 & +0.0387305 \\
\hline$\sigma_{A}$ & +0.0176615 & +0.0002679 & 0.0319108 & -0.0446594 & +0.0827357 \\
\hline$\sigma_{M}$ & +0.1055920 & +0.0002096 & 0.0336147 & +0.0399197 & +0.1682346 \\
\hline$\mu_{H}$ & +0.1216671 & -0.0004487 & 0.0335058 & +0.0583749 & +0.1891785 \\
\hline$\mu_{V}$ & -0.0390433 & -0.0011569 & 0.0330434 & -0.1020185 & +0.0305234 \\
\hline$\mu_{A}$ & +0.0176899 & +0.0013925 & 0.0323228 & -0.0455994 & +0.0791484 \\
\hline$\delta_{V}$ & +0.0038029 & -0.0010111 & 0.0340335 & -0.0611271 & +0.0690736 \\
\hline$\delta_{U}$ & +0.0532007 & -0.0004144 & 0.0324357 & -0.0115217 & +0.1187615 \\
\hline$\gamma_{U}$ & -0.2641840 & +0.0003395 & 0.0308615 & -0.3282315 & -0.2061550 \\
\hline$\gamma_{V}$ & -0.2422403 & -0.0005914 & 0.0328178 & -0.3056412 & -0.1780375 \\
\hline$\tau_{U}$ & -0.0734964 & -0.0015532 & 0.0319108 & -0.1341357 & -0.0135771 \\
\hline$\tau_{V}$ & +0.0115517 & -0.0025839 & 0.0312943 & -0.0466292 & +0.0706519 \\
\hline$\Pi_{H}$ & -0.3772186 & -0.0032223 & 0.0320140 & -0.4432971 & -0.3160064 \\
\hline$\phi_{A}$ & -0.4343937 & -0.0007132 & 0.0294558 & -0.4954435 & -0.3774096 \\
\hline$c_{A}$ & -0.8183722 & -0.0026977 & 0.0121513 & -0.8432971 & -0.7961376 \\
\hline$c_{L}$ & +0.4098845 & +0.0020502 & 0.0294757 & +0.3485913 & +0.4682076 \\
\hline$c_{B}$ & -0.6670218 & +0.0000797 & 0.0181261 & -0.7034560 & -0.6336552 \\
\hline$\kappa$ & +0.4298697 & -0.0008229 & 0.0302802 & +0.3714995 & +0.4885408 \\
\hline$\beta_{H V}$ & +0.4945893 & +0.0010630 & 0.0263685 & +0.4471908 & +0.5457829 \\
\hline$a_{M}$ & +0.2805036 & +0.0014569 & 0.0311481 & +0.2195653 & +0.3425459 \\
\hline$\epsilon_{V}$ & -0.3686739 & -0.0058365 & 0.0293159 & -0.4263527 & -0.3100787 \\
\hline$\omega_{V}$ & +0.2823186 & -0.0010166 & 0.0321963 & +0.2227506 & +0.3458825 \\
\hline$\eta_{U}$ & +0.0454152 & +0.0035739 & 0.0338749 & -0.0191098 & +0.1110642 \\
\hline$\eta_{V}$ & +0.0339887 & +0.0004213 & 0.0347059 & -0.0376288 & +0.0972901 \\
\hline$\eta_{I}$ & +0.3039816 & -0.0003942 & 0.0318035 & +0.2397632 & +0.3678769 \\
\hline$\xi_{V}$ & +0.5238138 & +0.0097502 & 0.0259621 & +0.4761949 & +0.5741889 \\
\hline
\end{tabular}

the PRCC is negative with value of -0.0381 in a constant temperature settings. For the use of adulticides, negative correlation with $R_{0 V}$ are obtained with PRCC values of $-0.8184,-0.7572,-0.6391,-0.7671$ and -0.6159 for temperature ranges of $15{ }^{\circ} \mathrm{C}-20^{\circ} \mathrm{C}, 20^{\circ} \mathrm{C}-25^{\circ} \mathrm{C}, 25^{\circ} \mathrm{C}-30{ }^{\circ} \mathrm{C}, 30^{\circ} \mathrm{C}-35^{\circ} \mathrm{C}$ and constant temperature, respectively.

Disease induced death rates, which have been shown to be main causes of backward bifurcations in mosquito borne diseases such as $[10,12,18,20,31]$ show wide variations as temperature changes for both non-vaccinated infected and vaccinated infected humans, PRCC values of $\delta_{V}=+0.0038, \delta_{V}=-0.0103, \delta_{V}=+0.0065, \delta_{V}=$ -0.0227 , and $\delta_{V}=+0.0318$ were obtained for temperature ranges of $15^{\circ} \mathrm{C}-20^{\circ} \mathrm{C}, 20^{\circ} \mathrm{C}-25^{\circ} \mathrm{C}, 25^{\circ} \mathrm{C}-30^{\circ} \mathrm{C}$, $30^{\circ} \mathrm{C}-35^{\circ} \mathrm{C}$ and constant respectively. Similarly for the same temperature ranges and constant, the PRCC values of $\delta_{U}=+0.0532, \delta_{U}=-0.0379, \delta_{U}=+0.0326, \delta_{U}=+0.0422$, and $\delta_{U}=+0.0325$ were respectively obtained. Variations occur in other temperature dependent and temperature independent parameters as presented in the Tables and Figures for the global sensitivity analysis.

\subsection{Numerical simulations}

We fix temperature values for the non-autonomous model (2.1), so that each of the temperature dependent parameter becomes constant. For simulation purposes, two different sets of parameter values that give $R_{0 V}=$ $0.297<1, N_{0}=14.2$ and $R_{0 V}=6.457>1, N_{0}=104.4$ were chosen within the ranges given in Table 2. Initial populations were chosen by $S_{U}(0)=2000 ; S_{V}(0)=1000 ; W_{U}(0)=1000 ; W_{V}(0)=500 ; E_{U}(0)=500$; 
TABLE 5. PRCC for parameters of the basic reproduction ratio with temperature of $20^{\circ} \mathrm{C}-25^{\circ} \mathrm{C}$.

\begin{tabular}{|c|c|c|c|c|c|}
\hline Parameter & Original & Bias & Std. error & Min. c.i. & Max. c.i. \\
\hline$\sigma_{U}$ & +0.0272416 & -0.0007902 & 0.0327054 & -0.0360532 & +0.0937781 \\
\hline$\sigma_{V}$ & -0.0073296 & -0.0006006 & 0.0328427 & -0.0699371 & +0.0585221 \\
\hline$\sigma_{A}$ & +0.0924912 & +0.0014401 & 0.0316656 & +0.0281105 & +0.1533272 \\
\hline$\sigma_{M}$ & +0.0944706 & -0.0000784 & 0.0325518 & +0.0332401 & +0.1623727 \\
\hline$\mu_{H}$ & +0.1127021 & +0.0000767 & 0.0316062 & +0.0482402 & +0.1711439 \\
\hline$\mu_{V}$ & -0.0200803 & -0.0008417 & 0.0328245 & -0.0830335 & +0.0488403 \\
\hline$\mu_{A}$ & -0.0566776 & -0.0001684 & 0.0329911 & -0.1215934 & +0.0070602 \\
\hline$\delta_{V}$ & -0.0102746 & -0.0017691 & 0.0340915 & -0.0700897 & +0.0622394 \\
\hline$\delta_{U}$ & -0.0379350 & +0.0007999 & 0.0340594 & -0.0300189 & +0.1049511 \\
\hline$\gamma_{U}$ & -0.2487736 & +0.0008432 & 0.0326861 & -0.3148316 & -0.1842316 \\
\hline$\gamma_{V}$ & -0.2480796 & -0.0003195 & 0.0310429 & -0.3157813 & -0.1869417 \\
\hline$\tau_{U}$ & -0.0495361 & -0.0009234 & 0.0334424 & -0.1146326 & +0.0179481 \\
\hline$\tau_{V}$ & +0.0197085 & -0.0015089 & 0.0331141 & -0.0439765 & +0.0853165 \\
\hline$\Pi_{H}$ & -0.3473466 & +0.0000988 & 0.0300609 & -0.4084650 & -0.2871868 \\
\hline$\phi_{A}$ & -0.0364459 & +0.0007974 & 0.0319624 & -0.1012336 & +0.0273710 \\
\hline$c_{A}$ & -0.7571855 & +0.0003732 & 0.0133595 & -0.7854990 & -0.7318509 \\
\hline$c_{L}$ & -0.0179992 & +0.0005700 & 0.0318969 & -0.0796521 & +0.0423534 \\
\hline$c_{B}$ & -0.6201653 & +0.0006721 & 0.0187960 & -0.6598916 & -0.5838745 \\
\hline$\kappa$ & +0.3893278 & -0.0013335 & 0.0307154 & +0.3278164 & +0.4527466 \\
\hline$\beta_{H V}$ & +0.4631499 & -0.0003937 & 0.0267489 & +0.4131450 & +0.5186893 \\
\hline$a_{M}$ & +0.1232240 & +0.0008872 & 0.0325427 & +0.0600620 & +0.1882611 \\
\hline$\epsilon_{V}$ & -0.3162192 & +0.0003416 & 0.0295568 & -0.3722439 & -0.2576412 \\
\hline$\omega_{V}$ & +0.2622113 & -0.0003436 & 0.0309711 & +0.2010132 & +0.3242911 \\
\hline$\eta_{U}$ & +0.0545866 & +0.0010445 & 0.0324711 & -0.0074782 & +0.1179109 \\
\hline$\eta_{V}$ & +0.0385358 & +0.0002086 & 0.0330906 & -0.0253969 & +0.1084278 \\
\hline$\eta_{I}$ & +0.2768270 & +0.0003028 & 0.0297059 & +0.2187766 & +0.3324457 \\
\hline$\xi_{V}$ & +0.4844347 & +0.0001834 & 0.0251479 & +0.4376538 & +0.5357899 \\
\hline
\end{tabular}

$E_{V}(0)=300 ; I_{U}(0)=200 ; I_{V}(0)=50 ; R_{U}(0)=150 ; R_{V}(0)=40 ; A_{M}(0)=2500 ; M_{U}(0)=1500 ; M_{E}(0)=$ $1000 ; M_{I}(0)=800$. In order to simulate the effect of seasonal variation, we use the generalized temperature function given by (2.2). Figures 6 and 7 show the solution profile of the model (2.1) for the total number of infectious humans $\left(I_{U}+I_{V}\right)$ when $R_{0 V}<1$ and when $R_{0 V}>1$, respectively. The solution profile of total infectious humans approach the DFE when $R_{0 V}<1$ (Fig. 6) and approach an endemic equilibrium when $R_{0 V}>1$ (Fig. 7). Similar results hold for the non-autonomous system for the case when $R_{0 T}<1$ and $R_{0 T}>1$ as presented in Figures 21A and 21B, respectively. Figure 22 shows effect of vaccination on disease incidence in humans with seasonal variation. The figure show that the oscillation pattern differs between the vaccinated and the non-vaccinated infectious (Fig. 22A) and exposed (Fig. 22B) humans, both in their subharmonic periods and the relative phase of cycles.

\section{Conclusion}

A new mathematical model to assess the effect of temperature on control strategies of malaria, is constructed and analysed. Some of the main findings of this study are summarized below:

(I) The autonomous mosquito-only model has a threshold quantity called the basic offspring number with the property that, if the threshold quantity $\left(N_{0}\right)$ is less than or equal to unity, the mosquito population goes to extinction, and it establishes if $\left(N_{0}\right)>1$. 
TABLE 6. PRCC for parameters of the basic reproduction ratio with temperature of $25^{\circ} \mathrm{C}-30{ }^{\circ} \mathrm{C}$.

\begin{tabular}{|c|c|c|c|c|c|}
\hline Parameter & Original & Bias & Std. error & Min. c.i. & Max. c.i. \\
\hline$\sigma_{U}$ & +0.0483804 & -0.0004057 & 0.0333765 & -0.0186168 & +0.1125817 \\
\hline$\sigma_{V}$ & +0.0114802 & -0.0011647 & 0.0309363 & -0.0532153 & +0.0730034 \\
\hline$\sigma_{A}$ & +0.0597791 & +0.0012013 & 0.0332133 & -0.0042205 & +0.1216877 \\
\hline$\sigma_{M}$ & +0.0445856 & -0.0004296 & 0.0327879 & -0.0216979 & +0.1082284 \\
\hline$\mu_{H}$ & +0.1068126 & -0.0004946 & 0.0321849 & +0.0445602 & +0.1664874 \\
\hline$\mu_{V}$ & -0.0645414 & -0.0009126 & 0.0331577 & -0.1262763 & +0.0088067 \\
\hline$\mu_{A}$ & -0.0559747 & +0.0003682 & 0.0329798 & -0.1186303 & +0.0069229 \\
\hline$\delta_{V}$ & +0.0064994 & -0.0015801 & 0.0336362 & -0.0546403 & +0.0768521 \\
\hline$\delta_{U}$ & +0.0326299 & +0.0003169 & 0.0335178 & -0.0318403 & +0.1004987 \\
\hline$\gamma_{U}$ & -0.2750038 & +0.0006289 & 0.0326875 & -0.3405111 & -0.2100418 \\
\hline$\gamma_{V}$ & -0.2766534 & -0.0002737 & 0.0313243 & -0.3389869 & -0.2153664 \\
\hline$\tau_{U}$ & -0.0510142 & -0.0006659 & 0.0335426 & -0.1156434 & +0.0156466 \\
\hline$\tau_{V}$ & +0.0060012 & -0.0001283 & 0.0326736 & -0.0553695 & +0.0731931 \\
\hline$\Pi_{H}$ & -0.3874218 & -0.0007302 & 0.0297809 & -0.4455378 & -0.3277662 \\
\hline$\phi_{A}$ & +0.0033342 & +0.0010076 & 0.0331209 & -0.0621007 & +0.0649226 \\
\hline$c_{A}$ & -0.6390782 & +0.0002818 & 0.0202658 & -0.6832559 & -0.6026203 \\
\hline$c_{L}$ & -0.1007759 & +0.0001927 & 0.0337962 & -0.1660626 & -0.0351183 \\
\hline$c_{B}$ & -0.6519635 & +0.0003658 & 0.0185159 & -0.6902679 & -0.6162609 \\
\hline$\kappa$ & +0.4161619 & -0.0017819 & 0.0299235 & +0.3567321 & +0.4768279 \\
\hline$\beta_{H V}$ & +0.5029739 & +0.0005889 & 0.0264660 & +0.4526908 & +0.5545998 \\
\hline$a_{M}$ & +0.0784946 & -0.0001587 & 0.0333855 & +0.0185815 & +0.1444017 \\
\hline$\epsilon_{V}$ & -0.3477630 & -0.0004523 & 0.0290541 & -0.4048732 & -0.2903114 \\
\hline$\omega_{V}$ & +0.3007633 & -0.0007619 & 0.0301747 & +0.2451951 & +0.3628431 \\
\hline$\eta_{U}$ & +0.0854023 & +0.0002371 & 0.0335750 & +0.0193157 & +0.1534979 \\
\hline$\eta_{V}$ & +0.0207561 & +0.0000308 & 0.0335975 & -0.0416263 & +0.0882889 \\
\hline$\eta_{I}$ & +0.3051394 & -0.0001698 & 0.0303403 & +0.2476859 & +0.3683635 \\
\hline$\xi_{V}$ & +0.4968967 & +0.0000247 & 0.0254239 & +0.4482705 & +0.5473321 \\
\hline
\end{tabular}

(II) The autonomous model version of system (2.1) has two disease-free equilibria, the mosquito-extinction equilibrium $\left(E_{2}\right)$ which is globally-asymptotically stable (GAS) when the basic offspring number $\left(N_{0}\right)$ is less than unity and the non-mosquito-extinction equilibrium $\left(E_{3}\right)$ which is locally asymptotically stable when $R_{0 V} \leq 1$.

(III) The autonomous model undergoes the phenomenon of backward bifurcation, which could be removed for a special case when malaria induced death rates $\left(\delta_{U}=0\right.$ and $\left.\delta_{V}=0\right)$ and the vaccine waning rate are negligible $\left(\omega_{V}=0\right)$.

(IV) Relationship between the vaccinated reproduction number and the type reproduction numbers is established, where it is shown that $T_{i}<1(i=1,2,3)$, provided $R_{0 V}<1$ (and $T_{i} \Leftrightarrow R_{0 V}$ ). This result suggest that, malaria can be control by targeting certain groups in the population.

(V) The non-autonomous model $(2.1)$ has a disease-free equilibrium $\left(E_{4}\right)$, which is shown to be locallyasymptotically stable whenever the associated reproduction ratio is less than unity and unstable otherwise.

(VI) The partial rank correlation coefficient for the use of larvicides is positively correlated with the vaccinated reproduction number when the temperature ranges between $15{ }^{\circ} \mathrm{C}-20^{\circ} \mathrm{C}$ and $30^{\circ} \mathrm{C}-35^{\circ} \mathrm{C}$, thus within those temperature intervals, use of larvicides may impede effort aimed at reducing malaria infection.

(VII) The successful use of adulticides $\left(c_{A}\right)$, bed nets $\left(c_{B}\right)$ and vaccine efficacy $\left(\epsilon_{V}\right)$ are negatively correlated with the vaccinated reproduction number within all the temperature ranges. 
TABLE 7. PRCC for parameters of the basic reproduction ratio with temperature of $30^{\circ} \mathrm{C}-35^{\circ} \mathrm{C}$.

\begin{tabular}{|c|c|c|c|c|c|}
\hline$\overline{\text { Parameter }}$ & Original & Bias & Std. error & Min. c.i. & Max. c.i. \\
\hline$\sigma_{U}$ & +0.0451980 & -0.0005944 & 0.03296141 & -0.0155342 & +0.1097078 \\
\hline$\sigma_{V}$ & +0.0080809 & -0.0014946 & 0.03216838 & -0.0561889 & +0.0695945 \\
\hline$\sigma_{A}$ & -0.0232658 & +0.0007003 & 0.03218964 & -0.0884070 & +0.0367905 \\
\hline$\sigma_{M}$ & +0.1068015 & -0.0000438 & 0.03214870 & +0.0438982 & +0.1708055 \\
\hline$\mu_{H}$ & +0.1069910 & -0.0004608 & 0.03147377 & +0.0463622 & +0.1691896 \\
\hline$\mu_{V}$ & -0.1075564 & -0.0004600 & 0.03417210 & -0.1743457 & -0.0394728 \\
\hline$\mu_{A}$ & +0.0040081 & +0.0001087 & 0.03221020 & -0.0606068 & +0.0692966 \\
\hline$\delta_{V}$ & -0.0226984 & -0.0014608 & 0.03351764 & -0.0894162 & +0.0425969 \\
\hline$\delta_{U}$ & +0.0422372 & +0.0004247 & 0.03338551 & -0.0233578 & +0.1107938 \\
\hline$\gamma_{U}$ & -0.2767289 & +0.0009461 & 0.03165590 & -0.3407932 & -0.2150720 \\
\hline$\gamma_{V}$ & -0.2379078 & -0.0005803 & 0.03091655 & -0.3003231 & -0.1780435 \\
\hline$\tau_{U}$ & -0.0707100 & -0.0003518 & 0.03182795 & -0.1300137 & -0.0067869 \\
\hline$\tau_{V}$ & +0.0068589 & -0.0008134 & 0.03346120 & -0.0575232 & +0.0741217 \\
\hline$\Pi_{H}$ & -0.4010288 & -0.0003367 & 0.02914214 & -0.4605973 & -0.3408796 \\
\hline$\phi_{A}$ & -0.0889220 & +0.0013691 & 0.03294498 & -0.1566463 & -0.0267232 \\
\hline$c_{A}$ & -0.7671031 & +0.0004052 & 0.01390989 & -0.7967973 & -0.7413719 \\
\hline$c_{L}$ & +0.1099999 & +0.0016757 & 0.03264374 & +0.0441499 & +0.1753972 \\
\hline$c_{B}$ & -0.6612125 & +0.0007278 & 0.01704738 & -0.6980249 & -0.6295967 \\
\hline$\kappa$ & +0.4223961 & -0.0016396 & 0.02881096 & +0.3652521 & +0.4794296 \\
\hline$\beta_{H V}$ & +0.5113951 & +0.0002744 & 0.02441461 & +0.4649900 & +0.5586622 \\
\hline$a_{M}$ & +0.0072425 & -0.0000582 & 0.03334982 & -0.0593828 & +0.0734986 \\
\hline$\epsilon_{V}$ & -0.3639675 & +0.0002173 & 0.02810343 & -0.4221400 & -0.3112162 \\
\hline$\omega_{V}$ & +0.2973694 & -0.0006766 & 0.03052003 & +0.2401928 & +0.3577935 \\
\hline$\eta_{U}$ & +0.0610768 & +0.0009632 & 0.03264673 & -0.0017988 & +0.1241164 \\
\hline$\eta_{V}$ & +0.0234672 & +0.0000817 & 0.03262804 & -0.0404243 & +0.0881068 \\
\hline$\eta_{I}$ & +0.3308712 & +0.0002972 & 0.02985926 & +0.2721758 & +0.3928036 \\
\hline$\xi_{V}$ & +0.5098077 & +0.0006601 & 0.02482458 & +0.4606819 & +0.5583210 \\
\hline
\end{tabular}

(VIII) For non-control parameters, the most positively correlated parameters within all ranges are the mosquito carrying capacity $(K)$, probability of disease transmission $\left(\beta_{H V}\right)$, reduction parameter in the transmission of infected vaccinated humans and rate of vaccination $\left(\xi_{V}\right)$. On the other hand, recovery rates $\gamma_{U}, \gamma_{V}$ and human recruitment rate $\left(\Pi_{H}\right)$ are the most negatively correlated in all the temperature ranges.

(IX) Numerical simulation of the model, using appropriate demographic and epidemiological data for Kwazulu Natal province of South Africa, show that (for the case where backward bifurcation does not occur), the hybrid strategy which combines all the strategies (that is combined use bed nets, vaccination and adulticides) is more effective than singular use of the aforementioned control strategies, that can lead to malaria elimination in the province.

(X) Seasonal variation may cause fluctuation in the number of people becoming infected with malaria in both the vaccinated and non-vaccinated populations.

(XI) Simulations of the model show that high vaccine efficacy is required to reduce the vaccinated reproduction number to a value below unity. Further, a singular effective use of bednets can result in effective control of malaria in a community provided the bed net coverage and the bed net efficacy are high enough (at least $60 \%$ each).

Acknowledgements. The authors acknowledges the support of South African DST/NRF SARChI chair on Mathematical Models and Methods in Bioengineering and Biosciences $\left(M^{3} B^{2}\right)$. The authors are grateful to Professor Abba B. Gumel for his very insightful and constructive comments in the formulation of the model. 
TABLE 8. PRCC for parameters of the basic reproduction ratio with constant.

\begin{tabular}{|c|c|c|c|c|c|}
\hline Parameter & Original & Bias & Std. error & Min. c.i. & Max. c.i. \\
\hline$\overline{\sigma_{U}}$ & +0.0537072 & -0.0000335 & 0.03142014 & -0.0065539 & +0.1154683 \\
\hline$\sigma_{V}$ & -0.0270326 & -0.0013361 & 0.03069721 & -0.0909801 & +0.0308928 \\
\hline$\sigma_{A}$ & +0.4461772 & +0.0000156 & 0.02649914 & +0.3933939 & +0.4959988 \\
\hline$\sigma_{M}$ & +0.3510576 & +0.0009426 & 0.03105527 & +0.2909725 & +0.4157925 \\
\hline$\mu_{H}$ & +0.1090479 & -0.0010750 & 0.03327486 & +0.0467657 & +0.1769173 \\
\hline$\mu_{V}$ & -0.1843498 & +0.0007244 & 0.03158123 & -0.2477469 & -0.1209180 \\
\hline$\mu_{A}$ & +0.0215627 & -0.0000023 & 0.03148113 & -0.0402444 & +0.0853352 \\
\hline$\delta_{V}$ & +0.0317568 & -0.0012651 & 0.03219245 & -0.0305051 & +0.1000426 \\
\hline$\delta_{U}$ & +0.0324881 & -0.0003282 & 0.03379430 & -0.0318886 & +0.0979736 \\
\hline$\gamma_{U}$ & -0.2687226 & +0.0001583 & 0.03116736 & -0.3283692 & -0.2040955 \\
\hline$\gamma_{V}$ & -0.2629236 & -0.0001416 & 0.03073967 & -0.3235758 & -0.2050292 \\
\hline$\tau_{U}$ & -0.0525523 & +0.0005959 & 0.03168751 & -0.1132575 & +0.0147820 \\
\hline$\tau_{V}$ & +0.0380073 & +0.0001263 & 0.03334126 & -0.0272727 & +0.1051935 \\
\hline$\Pi_{H}$ & -0.3428469 & -0.0007563 & 0.03105764 & -0.4012005 & -0.2843012 \\
\hline$\phi_{A}$ & +0.0174543 & -0.0002773 & 0.03422852 & -0.0476062 & +0.0827469 \\
\hline$c_{A}$ & -0.6159261 & +0.0004929 & 0.02160704 & -0.6576728 & -0.5746723 \\
\hline$c_{L}$ & -0.0381227 & +0.0020628 & 0.03327691 & -0.1092252 & +0.0236732 \\
\hline$c_{B}$ & -0.6559501 & +0.0002602 & 0.01775010 & -0.6918969 & -0.6237530 \\
\hline$\kappa$ & +0.4245334 & -0.0012985 & 0.02804146 & +0.3755547 & +0.4856456 \\
\hline$\beta_{H V}$ & +0.5049071 & +0.0003461 & 0.02369151 & +0.4600162 & +0.5533423 \\
\hline$a_{M}$ & +0.5617064 & +0.0004058 & 0.02354306 & +0.5165672 & +0.6088471 \\
\hline$\epsilon_{V}$ & -0.3690131 & -0.0001147 & 0.02917487 & -0.4256369 & -0.3135196 \\
\hline$\omega_{V}$ & +0.2531897 & -0.0001845 & 0.03223262 & +0.1924038 & +0.3192285 \\
\hline$\eta_{U}$ & +0.0561337 & +0.0008215 & 0.03287014 & -0.0142383 & +0.1240309 \\
\hline$\eta_{V}$ & +0.0144269 & -0.0013924 & 0.03449365 & -0.0490704 & +0.0864574 \\
\hline$\eta_{I}$ & +0.3183981 & +0.0001165 & 0.02926591 & +0.2595554 & +0.3769897 \\
\hline$\xi_{V}$ & +0.4727052 & +0.0002722 & 0.02628378 & +0.4225355 & +0.5231252 \\
\hline
\end{tabular}

\section{Appendix A. Proof of theorem 3.1}

We shall give the proof of the first part of Theorem 3.1 using similar approach to that in [4]. In particular, Theorem 6 of [4] reproduced below for convenience, will be used.

Theorem A.1. [4] Let $a, b \in \Omega^{*}$ be such that $a<b,[a, b] \subseteq \Omega^{*}$ and $f(b) \leq 0 \leq f(a)$. Then the system defines $a$ (positive) dynamical system on $[a, b]$. Moreover, if $[a, b]$ contains a unique equilibrium $q$ then $q$ is globally asymptotically stable on $[a, b]$.

Proof. To apply Theorem A.1 to system (3.1), let $q>0$ and let $A_{M q}$ be so large that the following inequalities hold:

$$
\begin{aligned}
& A_{M q} \geq q, \\
& M_{U q}=\frac{\left(\sigma_{A}+\mu_{A} \epsilon_{L} \alpha_{L}+\mu_{A}\right) A_{M q}}{\phi_{A}\left(1-c_{M}\right)} \geq q .
\end{aligned}
$$

Let $[a, b]=[0, b] \subseteq \mathbb{R}_{+}^{2}$, where $b=\left(A_{M q}, M_{U q}\right)^{\prime}$. It is easy to see that $f(0)=0$ and

$$
f(b)=\left(\begin{array}{c}
-\left[\sigma_{A}+\epsilon_{L} \alpha_{L}+\mu_{A}\right] \frac{A_{M q}^{2}}{K} \\
\sigma_{A} A_{M q}\left[1-\frac{1}{N_{0}}\right]
\end{array}\right)
$$


so that $f(b)<0$, provided $N_{0} \leq 1$.

Therefore $f(b) \leq 0 \leq f(0)$, whenever $N_{0}<1$. Thus the mosquito component of model (2.1) defines a positive dynamical system on $[0, b]$ and $E_{0}$ is GAS on $[0, b]$. But since $q$ is arbitrary, $b$ can be extended and the result holds on $\mathbb{R}_{+}^{2}$. The second part of the proof follows straightforward by linearization.

\section{Appendix B. Proof of theorem 4.1}

Proof. The proof is based on using centre manifold theory $[9,39]$. To apply the theorem, we carry out the following changes of variables. Let

$$
\begin{aligned}
& S_{U}=x_{1}, S_{V}=x_{2}, W_{U}=x_{3}, W_{V}=x_{4}, E_{U}=x_{5}, E_{V}=x_{6}, I_{U}=x_{7}, I_{V}=x_{8}, R_{U}=x_{9}, R_{V}=x_{10}, \\
& A_{M}=x_{11}, M_{U}=x_{12}, M_{E}=x_{13}, M_{I}=x_{14},
\end{aligned}
$$

so that,

$$
N_{H}=x_{1}+x_{2}+x_{3}+x_{4}+x_{5}+x_{6}+x_{7}+x_{8}+x_{9}+x_{10} \text { and } N_{V}=x_{12}+x_{13}+x_{14} .
$$

The transformed malaria model (2.1) is represented by

$$
\begin{aligned}
\frac{\mathrm{d} x_{1}}{\mathrm{~d} t} & =\Pi_{H}+\omega_{V} x_{2}-\xi_{V} x_{1}-\lambda_{H} x_{1}-\mu_{H} x_{1}, \\
\frac{\mathrm{d} x_{2}}{\mathrm{~d} t} & =\xi_{V} x_{1}-\lambda_{H}\left(1-\epsilon_{V}\right) x_{2}-\omega_{V} x_{2}-\mu_{H} x_{2}, \\
\frac{\mathrm{d} x_{3}}{\mathrm{~d} t} & =\tau_{U} x_{9}-\lambda_{H}\left(1-\epsilon_{W}\right) x_{3}-\mu_{H} x_{3}, \\
\frac{\mathrm{d} x_{4}}{\mathrm{~d} t} & =\tau_{V} x_{10}-\lambda_{H}\left(1-\epsilon_{W}\right) x_{4}-\mu_{H} x_{4}, \\
\frac{\mathrm{d} x_{5}}{\mathrm{~d} t} & =\lambda_{H} x_{1}+\lambda_{H}\left(1-\epsilon_{W}\right) x_{3}-\sigma_{U} x_{5}-\mu_{H} x_{5}, \\
\frac{\mathrm{d} x_{6}}{\mathrm{~d} t} & =\lambda_{H}\left(1-\epsilon_{V}\right) x_{2}+\lambda_{H}\left(1-\epsilon_{W}\right) x_{4}-\sigma_{V} x_{6}-\mu_{H} x_{6}, \\
\frac{\mathrm{d} x_{7}}{\mathrm{~d} t} & =\sigma_{U} x_{5}-\gamma_{U} x_{7}-\delta_{U} x_{7}-\mu_{H} x_{7}, \\
\frac{\mathrm{d} x_{8}}{\mathrm{~d} t} & =\sigma_{V} x_{6}-\gamma_{V} x_{8}-\delta_{V} x_{8}-\mu_{H} x_{8}, \\
\frac{\mathrm{d} x_{9}}{\mathrm{~d} t} & =\gamma_{U} x_{7}-\tau_{U} x_{9}-\mu_{H} x_{9}, \\
\frac{\mathrm{d} x_{10}}{\mathrm{~d} t} & =\gamma_{V} x_{8}-\tau_{V} x_{10}-\mu_{H} x_{10}, \\
\frac{\mathrm{d} x_{11}}{\mathrm{~d} t} & =\phi_{A}\left(1-\alpha_{L} \epsilon_{L}\right)\left(1-\frac{x_{11}}{K}\right) N_{V}-\sigma_{A} x_{11}-\mu_{A}\left(1+\alpha_{L} \epsilon_{L}\right) x_{11}, \\
\frac{\mathrm{d} x_{12}}{\mathrm{~d} t} & =\sigma_{A} x_{11}-\lambda_{V} x_{12}-\mu_{V}\left(1+\alpha_{A} \epsilon_{A}\right) x_{12}, \\
\frac{\mathrm{d} x_{13}}{\mathrm{~d} t} & =\lambda_{V} x_{12}-\sigma_{M} x_{13}-\mu_{V}\left(1+\alpha_{A} \epsilon_{A}\right) x_{13}, \\
\frac{\mathrm{d} x_{14}}{\mathrm{~d} t} & =\sigma_{M} x_{13}-\mu_{V}\left(1+\alpha_{A} \epsilon_{A}\right) x_{14},
\end{aligned}
$$


with the associated forces of infection given by

$$
\begin{aligned}
& \lambda_{H}=\frac{\beta_{H V} x_{14(t)}}{N_{H}}\left(1-\epsilon_{B} \alpha_{B}\right) a_{M} \\
& \text { and } \\
& \lambda_{V}=\frac{\beta_{H V}}{N_{H}(t)}\left(1-\epsilon_{B} \alpha_{B}\right) a_{M}\left(x_{7}+\eta_{I} x_{8}+\eta_{U} x_{9}+\eta_{V} x_{10}\right) .
\end{aligned}
$$

By letting $R_{0 V}=1$ we have,

$$
\frac{\beta_{H V}^{2} M_{U}^{*} \sigma_{M} a_{M}^{2}\left(1-\epsilon_{B} \alpha_{B}\right)^{2}}{\left(N_{H}^{*}\right)^{2} K_{10} K_{11}}\left(\frac{S_{U}^{*} \sigma_{U}}{K_{3} K_{5}}\left[1+\frac{\gamma_{U} \eta_{U}}{K_{7}}\right]+\frac{S_{V}^{*} \sigma_{V}\left(1-\epsilon_{V}\right)}{K_{4} K_{6}}\left[\eta_{I}+\frac{\gamma_{V} \eta_{V}}{K_{8}}\right]\right)=1
$$

suppose, further that $\beta_{H V}=\beta_{H V}^{*}$ is chosen to be the bifurcation parameter. The Jacobian matrix $\left(J^{*}\right)$ at the $\mathrm{DFE}$ with $\beta_{H V}=\beta_{H V}^{*}$ is given by

$$
J^{*}=\left[\begin{array}{ll}
P_{1} & P_{2} \\
P_{3} & P_{4}
\end{array}\right]
$$

where

$$
P_{1}=\left[\begin{array}{ccccccc}
-K_{1} & \omega_{V} & 0 & 0 & 0 & 0 & 0 \\
\xi_{V} & -K_{2} & 0 & 0 & 0 & 0 & 0 \\
0 & 0 & -\mu_{H} & 0 & 0 & 0 & 0 \\
0 & 0 & 0 & -\mu_{H} & 0 & 0 & 0 \\
0 & 0 & 0 & 0 & -K_{3} & 0 & 0 \\
0 & 0 & 0 & 0 & 0 & -K_{4} & 0 \\
0 & 0 & 0 & 0 & \sigma_{U} & 0 & -K_{5} \\
& & & & & &
\end{array}\right]
$$

$$
P_{2}=\left[\begin{array}{ccccccc}
0 & 0 & 0 & 0 & 0 & 0 & -\frac{\beta_{H V}^{*} S_{U}^{*}\left(1-\epsilon_{B} \alpha_{B}\right)}{N_{H}^{*}} \\
0 & 0 & 0 & 0 & 0 & 0 & -\frac{\beta_{H V}^{*} S_{V}^{*}\left(1-\epsilon_{B} \alpha_{B}\right)\left(1-\epsilon_{V}\right)}{N_{H}^{*}} \\
0 & \tau_{U} & 0 & 0 & 0 & 0 & 0 \\
0 & 0 & \tau_{V} & 0 & 0 & 0 & 0 \\
0 & 0 & 0 & 0 & 0 & 0 & \frac{\beta_{H V}^{*} S_{U}^{*}\left(1-\epsilon_{B} \alpha_{B}\right)}{N_{H}^{*}} \\
0 & 0 & 0 & 0 & 0 & 0 & \frac{\beta_{H V}^{*} S_{V}^{*}\left(1-\epsilon_{B} \alpha_{B}\right)\left(1-\epsilon_{V}\right)}{N_{H}^{*}} \\
0 & 0 & 0 & 0 & 0 & 0 & 0
\end{array}\right]
$$




$$
P_{3}=\left[\begin{array}{ccccccc}
0 & 0 & 0 & 0 & 0 & \sigma_{V} & 0 \\
0 & 0 & 0 & 0 & 0 & 0 & \gamma_{U} \\
0 & 0 & 0 & 0 & 0 & 0 & 0 \\
0 & 0 & 0 & 0 & 0 & 0 & 0 \\
0 & 0 & 0 & 0 & 0 & 0 & -\frac{\beta_{H V}^{*} M_{U}^{*}\left(1-\epsilon_{B} \alpha_{B}\right)}{N_{H}^{*}} \\
0 & 0 & 0 & 0 & 0 & 0 & \frac{\beta_{H V}^{*} M_{U}^{*}\left(1-\epsilon_{B} \alpha_{B}\right)}{N_{H}^{*}} \\
0 & 0 & 0 & 0 & 0 & 0 & 0
\end{array}\right]
$$

and

$$
P_{4}=\left[\begin{array}{ccccccc}
-K_{6} & 0 & 0 & 0 & 0 & 0 & 0 \\
0 & -K_{7} & 0 & 0 & 0 & 0 & 0 \\
\gamma_{V} & 0 & -K_{8} & 0 & 0 & 0 & 0 \\
0 & 0 & 0 & -K_{9} N_{0} & \frac{\phi_{V}}{N_{0}} & \frac{\phi_{V}}{N_{0}} & \frac{\phi_{V}}{N_{0}} \\
-Q_{A} \eta_{I} & -Q_{A} \eta_{U} & -Q_{A} \eta_{V} & \sigma_{A} & -K_{10} & 0 & 0 \\
Q_{A} \eta_{I} & Q_{A} \eta_{U} & Q_{A} \eta_{V} & 0 & 0 & -K_{11} & 0 \\
0 & 0 & 0 & 0 & 0 & \sigma_{M} & -K_{10}
\end{array}\right]
$$

where $Q_{A}=\frac{\beta_{H V}^{*} M_{U}^{*}\left(1-\epsilon_{B} \alpha_{B}\right)}{N_{H}^{*}}$.

The Jacobian matrix $\left(J^{*}\right)$ of the linearized system has a simple zero eigenvalue (with all other eigenvalues having negative real parts). Hence the theory based on Center Manifold Theory [9, 39] can be used to analysed the dynamics of the system (B.1). We obtained the left eigenvector $(v)$ corresponding to the zero eigenvalue denoted by $v=\left[v_{1}, v_{2}, v_{3}, v_{4}, v_{5}, v_{6}, v_{7}, v_{8}, v_{9}, v_{10}, v_{11}, v_{12}, v_{13}, v_{14}\right]^{T}$, where

$$
\begin{aligned}
v_{1}= & 0, \quad v_{2}=0, \quad v_{3}=0, \quad v_{4}=0, \quad v_{5}=\frac{\beta_{H V}^{*} M_{U}^{*} \sigma_{M} \sigma_{U}\left(1-\epsilon_{B} \alpha_{B}\right)}{K_{3} K_{5} K_{11} N_{H}^{*}}\left(1+\frac{\gamma_{U} \eta_{U}}{K_{7}}\right) v_{14}, \\
v_{6}= & \frac{\beta_{H V}^{*} M_{U}^{*} \sigma_{M} \sigma_{V}\left(1-\epsilon_{B} \alpha_{B}\right)}{K_{4} K_{6} K_{11} N_{H}^{*}}\left(\eta_{I}+\frac{\gamma_{V} \eta_{V}}{K_{8}}\right) v_{14}, \quad v_{7}=\frac{\beta_{H V}^{*} M_{U}^{*} \sigma_{M}\left(1-\epsilon_{B} \alpha_{B}\right)}{K_{5} K_{11} N_{H}^{*}}\left(1+\frac{\gamma_{U} \eta_{U}}{K_{7}}\right) v_{14}, \\
v_{8}= & \frac{\beta_{H V}^{*} M_{U}^{*} \sigma_{M}\left(1-\epsilon_{B} \alpha_{B}\right)}{K_{6} K_{11} N_{H}^{*}}\left(\eta_{I}+\frac{\gamma_{V} \eta_{V}}{K_{8}}\right) v_{14}, \quad v_{9}=\frac{\beta_{H V}^{*} M_{U}^{*} \sigma_{M} \eta_{U}\left(1-\epsilon_{B} \alpha_{B}\right)}{N_{H}^{*} K_{7} K_{11}} v_{14}, \\
v_{10}= & \frac{\beta_{H V}^{*} M_{U}^{*} \sigma_{M} \eta_{V}\left(1-\epsilon_{B} \alpha_{B}\right)}{N_{H}^{*} K_{8} K_{11}} v_{14}, \quad v_{11}=0, \quad v_{12}=0, \quad v_{13}=\frac{\sigma_{M}}{K_{11}} v_{14} \quad v_{14}=\left(\beta_{H V}^{*}\right)^{2}\left(1-\alpha_{B} \epsilon_{B}\right)^{2} \\
& \times M_{U}^{*} \sigma_{M}\left(S_{U}^{*} \sigma_{U} Q_{11}+S_{V}^{*} \sigma_{V}\left(1-\epsilon_{V}\right) Q_{12}\right)+\left(N_{H}^{*}\right)^{2} K_{11}\left[K_{10}+K_{11}\right]\left(K_{3} K_{4} K_{5} K_{6} K_{7} K_{8}\right)^{2}
\end{aligned}
$$

with $Q_{11}=\left[K_{7}\left(K_{3}+K_{5}\right)\left(K_{7}+\gamma_{U} \eta_{U}\right)+K_{3} K_{5} \gamma_{U} \eta_{U}\right]\left(K_{4} K_{6} K_{8}\right)^{2}, Q_{12}=\left[K_{8}\left(K_{4}+K_{6}\right)\left(K_{8} \eta_{I}+\gamma_{V} \eta_{V}\right)+\right.$ $\left.K_{4} K_{6} \gamma_{V} \eta_{V}\right]\left(K_{3} K_{5} K_{7}\right)^{2}$. 
The right eigenvector $(w)$ is denoted by $w=\left[w_{1}, w_{2}, w_{3}, w_{4}, w_{5}, w_{6}, w_{7}, w_{8}, w_{9}, w_{10}, w_{11}, w_{12}, w_{13}, w_{14}\right]^{T}$, where

$$
\begin{aligned}
& w_{1}=\frac{-\beta_{H V}^{*}\left(1-\epsilon_{B} \alpha_{B}\right)\left(S_{U}^{*} K_{2}+S_{V}^{*} \omega_{V}\left(1-\epsilon_{V}\right)\right)}{N_{H}^{*}\left(K_{1} K_{2}-\omega_{V} \xi_{V}\right)} w_{14}, \\
& w_{2}=\frac{-\beta_{H V}^{*}\left(1-\epsilon_{B} \alpha_{B}\right)\left(S_{U}^{*} \xi_{V}+S_{V}^{*} K_{1}\left(1-\epsilon_{V}\right)\right)}{N_{H}^{*}\left(K_{1} K_{2}-\omega_{V} \xi_{V}\right)} w_{14}, \\
& w_{3}=\frac{\beta_{H V}^{*} S_{U}^{*} \sigma_{U} \gamma_{U} \tau_{U}\left(1-\epsilon_{B} \alpha_{B}\right)}{N_{H}^{*} K_{3} K_{5} K_{7} \mu_{H}} w_{14}, \quad w_{4}=\frac{\beta_{H V}^{*} S_{V}^{*} \sigma_{U} \gamma_{V} \tau_{V}\left(1-\epsilon_{B} \alpha_{B}\right)\left(1-\epsilon_{V}\right)}{N_{H}^{*} K_{4} K_{6} K_{8} \mu_{H}} w_{14}, \\
& w_{5}=\frac{\beta_{H V}^{*} S_{U}^{*}\left(1-\epsilon_{B} \alpha_{B}\right)}{N_{H}^{*} K_{3}} w_{14}, \quad w_{6}=\frac{\beta_{H V}^{*} S_{V}^{*}\left(1-\epsilon_{B} \alpha_{B}\right)\left(1-\epsilon_{V}\right)}{N_{H}^{*} K_{4}} w_{14}, \\
& w_{7}=\frac{\beta_{H V}^{*} S_{U}^{*} \sigma_{U}\left(1-\epsilon_{B} \alpha_{B}\right)}{N_{H}^{*} K_{3} K_{5}} w_{14}, \quad w_{8}=\frac{\beta_{H V}^{*} S_{V}^{*} \sigma_{V}\left(1-\epsilon_{B} \alpha_{B}\right)\left(1-\epsilon_{V}\right)}{N_{H}^{*} K_{4} K_{6}} w_{14}, \\
& w_{9}=\frac{\beta_{H V}^{*} S_{U}^{*} \sigma_{U} \gamma_{U}\left(1-\epsilon_{B} \alpha_{B}\right)}{N_{H}^{*} K_{3} K_{7} K_{5}} w_{14}, \quad w_{10}=\frac{\beta_{H V}^{*} S_{V}^{*} \sigma_{V} \gamma_{V}\left(1-\epsilon_{B} \alpha_{B}\right)\left(1-\epsilon_{V}\right) w_{14}}{N_{H}^{*} K_{4} K_{6} K_{8}}, \quad w_{11}=0, \\
& w_{12}=\frac{K_{11}}{\sigma_{M}} w_{14}, \quad w_{13}=\frac{K_{10}}{\sigma_{M}} w_{14}, \quad w_{14}=\left(N_{H}^{*}\right)^{2} K_{11}\left(K_{3} K_{4} K_{5} K_{6} K_{7} K_{8}\right)^{2},
\end{aligned}
$$

The eigenvalues $v_{14}$ and $w_{14}$ are chosen so that the classical requirement that the dot product of $v$ and $w$ satisfies $v \cdot w=1$. Clearly $v_{i} \geq 0$ while $w_{1}, w_{2}$ are negative (for variables that are non-zero at DFE), such choice is justified by Remark 1 of [9] which states;

Remark B.1. The requirement that $w$ is non-negative in the theorem is not necessary. When some components in $w$ are negative, we still can apply this theorem, but one has to compare $w$ with the actual equilibrium because the general parametrization of the Centre Manifold before the coordinate change is,

$$
W^{c}=\left\{x_{0}+c(t) w+h\left(c, \phi_{V}\right): v \cdot h\left(c, \phi_{V}\right)=0,|c| \leq c_{0}, c(0)=0\right\}
$$

provided that $x_{0}$ is a non-negative equilibrium of interest (usually $x_{0}$ is the disease-free equilibrium). Hence, $x_{0}-\frac{2 b \phi_{V}}{a}>0$ requires that $w_{j}>0$ whenever $x_{0}(j)=0$. If $x_{0}(j)>0$, then $w(j)$ need not be positive [9].

It can be verified that $v \cdot w=1$, thus all the necessarily conditions for the application of Lemma 3 and Theorem 4 of [39] as well as Theorem 4.1 of [9] are satisfied. After series of computations and simplifications we obtained

$$
\begin{aligned}
\mathbf{a}= & \sum_{k, i, j=1}^{n} v_{k} w_{I} w_{j} \frac{\partial^{2} f_{k}}{\partial x_{I} \partial x_{j}}(0,0)=-\frac{2\left(\beta_{H V}^{*}\right)^{2}\left(1-\epsilon_{B} \alpha_{B}\right)^{2} w_{14}^{2} v_{14}}{\left(N_{H}^{*}\right)^{2}}\left\{\frac{M_{U}^{*} P_{1}\left(1-\epsilon_{B} \alpha_{B}\right) \sigma_{M}}{K_{11}}\left(P_{3}+P_{5}-P_{6}-P_{7}\right)\right. \\
& +N_{H}^{*} P_{1}+P_{9} S_{V}^{*}\left(1-\epsilon_{B} \alpha_{B}\right)\left(1-\epsilon_{V}\right)\left(P_{3}+P_{4}-P_{6}\right)+P_{8} S_{U}^{*}\left(1-\epsilon_{B} \alpha_{B}\right)\left(P_{2}+P_{5}-P_{7}\right) \\
& +P_{8} P_{10}\left(1-\epsilon_{B} \alpha_{B}\right)\left(N_{H}^{*} \epsilon_{W}-S_{V}^{*}\right)+P_{9} P_{11}\left(1-\epsilon_{B} \alpha_{B}\right)\left(N_{H}^{*} \epsilon_{W}-S_{U}^{*}\right)+P_{9} P_{11} S_{V}^{*}\left(1-\epsilon_{B} \alpha_{B}\right) \epsilon_{V} \\
& \left.+P_{8} P_{6} S_{V}^{*}\left(1-\epsilon_{B} \alpha_{B}\right)+P_{9} P_{7} S_{U}^{*}\left(1-\epsilon_{B} \alpha_{B}\right)\left(1-\epsilon_{V}\right)\right\}
\end{aligned}
$$


where,

$$
\begin{aligned}
& P_{1}=\frac{S_{U}^{*} \beta_{H V}^{*} \sigma_{U}}{N_{H}^{*} K_{3} K_{5}}\left(1+\frac{\gamma_{U} \eta_{U}}{K_{7}}\right)+\frac{S_{V}^{*} \beta_{H V}^{*} \sigma_{V}\left(1-\epsilon_{V}\right)}{N_{H}^{*} K_{4} K_{6}}\left(\eta_{I}+\frac{\gamma_{V} \eta_{V}}{K_{8}}\right), \quad P_{2}=\frac{S_{U}^{*} \beta_{H V}^{*}}{N_{H}^{*} K_{3}}\left(1+\frac{\sigma_{U}}{K_{5}}+\frac{\sigma_{U} \gamma_{U}}{K_{5} K_{7}}\right), \\
& P_{3}=\frac{S_{U}^{*} \beta_{H V}^{*}}{N_{H}^{*} K_{3}}\left(1+\frac{\sigma_{U}}{K_{5}}+\frac{\sigma_{U} \gamma_{U}}{K_{5} K_{7}}+\frac{\tau_{U} \sigma_{U} \gamma_{U}}{K_{5} K_{7} \mu_{H}}\right), \quad P_{4}=\frac{S_{V}^{*} \beta_{H V}^{*}\left(1-\epsilon_{V}\right)}{N_{H}^{*} K_{4}}\left(1+\frac{\sigma_{V}}{K_{6}}+\frac{\sigma_{V} \gamma_{V}}{K_{6} K_{8}}\right), \\
& P_{5}=\frac{S_{V}^{*} \beta_{H V}^{*}\left(1-\epsilon_{V}\right)}{N_{H}^{*} K_{4}}\left(1+\frac{\sigma_{V}}{K_{6}}+\frac{\sigma_{V} \gamma_{V}}{K_{6} K_{8}}+\frac{\tau_{V} \sigma_{V} \gamma_{V}}{K_{6} K_{8} \mu_{H}}\right), \quad P_{6}=\frac{\beta_{H V}^{*}\left(S_{U}^{*} K_{2}+S_{V}^{*} \omega_{V}\left(1-\epsilon_{V}\right)\right)}{N_{H}^{*}\left(K_{1} K_{2}-\omega_{V} \xi_{V}\right)}, \\
& P_{7}=\frac{\beta_{H V}^{*}\left(S_{U}^{*} \xi_{V}+S_{V}^{*} K_{1}\left(1-\epsilon_{V}\right)\right)}{N_{H}^{*}\left(K_{1} K_{2}-\omega_{V} \xi_{V}\right)}, \quad P_{8}=\frac{\beta_{H V}^{*} M_{U}^{*} \sigma_{M} \sigma_{U}}{K_{3} K_{5} K_{11} N_{H}^{*}}\left(1+\frac{\gamma_{U} \eta_{U}}{K_{7}}\right), \\
& P_{9}=\frac{\beta_{H V}^{*} M_{U}^{*} \sigma_{M} \sigma_{V}}{K_{4} K_{6} K_{11} N_{H}^{*}}\left(\eta_{I}+\frac{\gamma_{V} \eta_{V}}{K_{8}}\right), \quad P_{10}=\frac{\beta_{H V}^{*} S_{U}^{*} \sigma_{U} \gamma_{U} \tau_{U}}{N_{H}^{*} K_{3} K_{5} K_{7} \mu_{H}}, \quad P_{11}=\frac{\beta_{H V}^{*} S_{V}^{*} \sigma_{U} \gamma_{V} \tau_{V}\left(1-\epsilon_{V}\right)}{N_{H}^{*} K_{4} K_{6} K_{8} \mu_{H}},
\end{aligned}
$$

and

$$
\begin{aligned}
\mathbf{b}= & \sum_{k, i=1}^{n} v_{k} w_{I} \frac{\partial^{2} f_{k}}{\partial x_{I} \partial \phi_{V}}(0,0)=\frac{\beta_{H V}^{*} M_{U}^{*} \sigma_{M} v_{14} w_{14}}{\left(N_{H}^{*}\right)^{2}}\left[\frac{S_{U}^{*} \sigma_{U}}{K_{3} K_{5} K_{11}}\left(1+\frac{\gamma_{U} \eta_{U}}{K_{7}}\right)+\frac{S_{V}^{*} \sigma_{V}}{K_{4} K_{6} K_{11}}\left(\eta_{I}+\frac{\gamma_{V} \eta_{V}}{K_{8}}\right)\right] \\
& +\left[\frac{\beta_{H V}^{*} S_{U}^{*} \sigma_{M} \gamma_{U} \sigma_{U}}{N_{H}^{*} K_{3} K_{5} K_{7}}\left(1+\frac{\gamma_{U} \eta_{U}}{K_{5}}\right)+\frac{\beta_{H V}^{*} S_{V}^{*} \sigma_{M} \sigma_{V}\left(1-\epsilon_{V}\right)}{N_{H}^{*} K_{4} K_{6}}\left(\eta_{I}+\frac{\gamma_{V} \eta_{V}}{K_{8}}\right)\right] \frac{M_{U}^{*} \sigma_{M} v_{14} w_{14}}{N_{H}^{*} K_{11}}>0
\end{aligned}
$$

Since $\mathbf{b}>0$, the direction of bifurcation depends on the sign of $\mathbf{a}$, which can be positive or negative, and $\mathbf{a}>0$ means the model (2.1) undergoes backward bifurcation at $R_{0 V}=1[9,39]$.

\section{REFERENCES}

[1] A. Abdelrazec and A.B. Gumel, Mathematical assessment of the role of temperature and rainfall on mosquito population dynamics. J. Math. Biol. 742017 1351-1395.

[2] F.B. Agusto, A.B. Gumel and P.E. Parham, Qualitative assessment of the role of temperature variations on malaria transmission dynamics. J. Biolog. Syst. 23 (2015) 1550030.

[3] D. Alonso, M.J. Bouma and M. Pascual, Epidemic malaria and warmer temperatures in recent decades in an East African highland. Proc. Roy. Soc. Lond. B 278 (2011) 1661-1669.

[4] R. Anguelov, Y. Dumont and J. Lubuma, Mathematical modeling of sterile insect technology for control of anopheles mosquito. Comput. Math. Appl. 64 (2012) 374-389.

[5] J.I. Blanford, S. Blanford, R.G. Crane, M.E. Mann, K.P. Paaijmans, K.V. Schreiber and M.B. Thomas, Implications of temperature variation for malaria parasite development across Africa. Sci. Rep. 3 (2013) 1300.

[6] J.F. Briere, P. Pracros, A.Y. Le Roux and J.S. Pierre, A novel rate model of temperature-dependent development for arthropods. Environ. Entomol. 28 (1999) 22-29.

[7] T.M. Bury, C.T. Bauch and M. Anand, Charting pathways to climate change mitigation in a coupled socio-climate model. PLoS Comput. Biol. 15 (2019) e1007000.

[8] C. Caminade, S. Kovats, J. Rocklov, A.M. Tompkins, A.P. Morse, F.J. Colón-González, H. Stenlund, P. Martens and S.J. Lloyd, Impact of climate change on global malaria distribution. Proc. Natl. Acad. Sci. 111 (2014) 3286-3291.

[9] C. Castillo-Chavez and B. Song, Dynamical models of tuberculosis and their applications. Math. Biosci. Eng. 2 (2004) 361-404.

[10] N. Chitnis, J.M. Cushing and J.M. Hyman, Bifurcation analysis of a mathematical model for malaria transmission. SIAM J. Appl. Math. 67 (2006) 24-45.

[11] U.A. Danbaba and S.M. Garba, Analysis of model for the transmission dynamics of Zika with sterile insect technique. In: Mathematical Methods and Models in Biosciences, edited by R. Anguelov, M. Lachowicz. Biomath Forum, Sofia, GNU General Public License, University British Columbia, Canada (2018) 81-99. https://doi.org/10.11145/texts.2018.01.083

[12] U.A. Danbaba and S.M. Garba, Modeling the transmission dynamics of Zika with sterile insect technique. Math. Methods Appl. Sci. 41 (2018) 8871-8896.

[13] B. Dembele, A. Friedman and A.A. Yakubu, Malaria model with periodic mosquito birth and death rates. J. Biol. Dyn. 3 (2009) 430-445.

[14] Y. Dumont, F. Chiroleu and C. Domerg, On a temporal model for the Chikungunya disease: modeling, theory and numerics. Math. Biosci. 213 (2008) 80-91. 
[15] Y. Dumont and F. Chiroleu, Vector control for the Chikungunya disease. Math. Biosci. Eng. 7 (2010) 313-345.

[16] J. Dushoff, J.B. Plotkin, S.A. Levin and D.J. Earn, Dynamical resonance can account for seasonality of influenza epidemics. Proc. Natl. Acad. Sci. 101 (2004) 16915-16916.

[17] S.E. Eikenberry and A.B. Gumel, Mathematical modeling of climate change and malaria transmission dynamics: a historical review. J. Math. Biol. (2018) 1-77.

[18] S.M. Garba, A.B. Gumel and M.A. Bakar, Backward bifurcations in dengue transmission dynamics. Math. Biosci. 215 (2008) $11-25$.

[19] S.M. Garba and A.B. Gumel, Effect of cross-immunity on the transmission dynamics of two strains of dengue. Int. J. Comput. Math. 87 (2010) 2361-2384.

[20] S.M. Garba and M.A. Safi, Mathematical analysis of West Nile virus model with discrete delays. Acta Math. Sci. 33 (2013) 1439-1462.

[21] D. Greenhalgh and I.A. Moneim, SIRS epidemic model and simulations using different types of seasonal contact rate. Syst. Anal. Model. Simul. 43 (2010) 573-600.

[22] P.W. Gething, T.P. Van Boeckel, D.L. Smith, C.A. Guerra, A.P. Patil, R.W. Snow and S.I. Hay, Modelling the global constraints of temperature on transmission of Plasmodium falciparum and P. vivax. Parasites Vectors 4 (2011) 92.

[23] J.A. Heesterbeek and M.G. Roberts, The type-reproduction number T in models for infectious disease control. Math. Biosci. 206 (2007) 3-10.

[24] V. Laperriere, K. Brugger and F. Rubel, Simulation of the seasonal cycles of bird, equine and human West Nile virus cases. Prevent. Veter. Med. 98 (2011) 99-110.

[25] Y. Lou and X.Q. Zhao, A climate-based malaria transmission model with structured vector population. SIAM J. Appl. Math. 70 (2010) 2023-2044.

[26] I.A. Moneim and Greenhalgh, Use of a periodic vaccination strategy to control the spread of epidemics with seasonally varying contact rate. Math. Biosci. Eng. 2 (2005) 591-611.

[27] E.A. Mordecai, K.P. Paaijmans, L.R. Johnson, C. Balzer, T. Ben-Horin, E. de Moor, A. McNally, S. Pawar, S.J. Ryan, T.C. Smith and K.D. Lafferty, Optimal temperature for malaria transmission is dramatically lower than previously predicted. Ecol. Lett. 16 (2013) 22-30.

[28] C.C. Murdock, E.D. Sternberg and M.B. Thomas, Malaria transmission potential could be reduced with current and future climate change. Sci. Rep. 6 (2016) 27771.

[29] G.A. Ngwa and W.S. Shu, A mathematical model for endemic malaria with variable human and mosquito populations. Math. Comput. Model. 32 (2000) 747-763.

[30] A.M. Niger and A.B. Gumel, Mathematical analysis of the role of repeated exposure on malaria transmission dynamics. Differ. Equ. Dyn. Syst. 16 (2008) 251-287.

[31] K. Okuneye and A.B. Gumel, Analysis of a temperature-and rainfall-dependent model for malaria transmission dynamics. Math. Biosci. 287 (2017) 72-92.

[32] K. Okuneye, S.E. Eikenberry and A.B. Gumel, Weather-driven malaria transmission model with gonotrophic and sporogonic cycles. J. Biol. Dyn. 13 (2019) 288-324.

[33] S. Olaniyi, K.O. Okosun, O. Adesanya and E.A. Areo, Global stability and optimal control analysis of malaria dynamics in the presence of human travelers. 10 (2018) 166-186.

[34] P.E. Parham, J. Waldock, G.K. Christophides, D. Hemming, F. Agusto, K.J. Evans, N. Fefferman, H. Gaff, A. Gumel, S. LaDeau and S. Lenhart, Climate, environmental and socio-economic change: weighing up the balance in vector-borne disease transmission. Philo. Trans. R. Soc. B 370 (2015) 20130551.

[35] M.A. Penny, R. Verity, C.A. Bever, C. Sauboin, K. Galactionova, S. Flasche, M.T. White, E.A. Wenger, N. Van de Velde, P. Pemberton-Ross and J.T. Griffin, Public health impact and cost-effectiveness of the RT,S/AS01 malaria vaccine: a systematic comparison of predictions from four mathematical models. The Lancet 387 (2016) 367-375.

[36] F. Rubel, K. Brugger, M. Hantel, S. Chvala-Mannsberger, T. Bakonyi, H. Weissenböck and N. Nowotny, Explaining Usutu virus dynamics in Austria: model development and calibration. Prev. Veter. Med. 85 (2008) 166-186.

[37] J. Shaman, M. Spiegelman, M. Cane and M. Stieglitz, A hydrologically driven model of swamp water mosquito population dynamics. Ecol. Model. 194 (2006) 395-404.

[38] R. Tuteja, Malaria-an overview. FEBS J. 274 (2007) 4670-4679.

[39] P. Van den Driessche and J. Watmough, Reproduction numbers and sub-threshold endemic equilibria for compartmental models of disease transmission. Math. Biosci. 180 (2002) 29-48.

[40] W. Wang and X.Q. Zhao, Threshold dynamics for compartmental epidemic models in periodic environments. J. Dyn. Differ. Equ. 20 (2008) 699-717.

[41] M.T. White, R. Verity, T.S. Churcher and A.C. Ghani, Vaccine approaches to malaria control and elimination: Insights from mathematical models. Vaccine 33 (2015) 7544-7550.

[42] Malaria: World Health Organization fact-sheets. Available at http://www.who.int/news-room/fact-sheets/detail/malaria accessed on 1st August (2018).

[43] H.M. Yang, A mathematical model for malaria transmission relating global warming and local socioeconomic conditions. Rev. Saude Pub. 35 (2001) 224-231. 